\title{
The Cahn-Hilliard-Hele-Shaw System with Singular Potential
}

\author{
Andrea Giorgini, Maurizio Grasselli ${ }^{\dagger}$ HaO Wu $\mathrm{W}^{\ddagger}$
}

June 16, 2017

\begin{abstract}
The Cahn-Hilliard-Hele-Shaw system is a fundamental diffuse-interface model for an incompressible binary fluid confined in a Hele-Shaw cell. It consists of a convective Cahn-Hilliard equation in which the velocity $\boldsymbol{u}$ is subject to a Korteweg force through Darcy's equation. In this paper, we aim to investigate the system with a physically relevant potential (i.e., of logarithmic type). This choice ensures that the (relative) concentration difference $\varphi$ takes values within the admissible range. To the best of our knowledge, essentially all the available contributions in the literature are concerned with a regular approximation of the singular potential. We first prove the existence of a global weak solution with finite energy that satisfies an energy dissipative property. Then, in dimension two, we further obtain the uniqueness and regularity of global weak solutions. In particular, we show that any two-dimensional weak solution satisfies the so-called strict separation property, namely, if $\varphi$ is not a pure state at some initial time, then it stays instantaneously away from the pure states. When the spatial dimension is three, we prove the existence of a unique global strong solution, provided that the initial datum is regular enough and sufficiently close to any local minimizer of the free energy. This also yields the local Lyapunov stability of the local minimizer itself. Finally, we prove that under suitable assumptions any global solution converges to a single equilibrium as time goes to infinity.
\end{abstract}

Keywords: Cahn-Hilliard equation, Darcy's equation, singular potential, well-posedness, long-time behavior.

MSC2010: 35B40, 35B41, 35Q35, 76D27.

\section{Introduction}

Topological transitions of interfaces between macroscopically immiscible fluids are essential phenomena in fluid dynamics. Typical examples of such changes in the multicomponent fluid topology are pinch-off and reconnection (see, e.g., [25]). Through the

\footnotetext{
*Dipartimento di Matematica, Politecnico di Milano, Milano 20133, Italy, andrea.giorgini@polimi.it

${ }^{\dagger}$ Dipartimento di Matematica, Politecnico di Milano, Milano 20133, Italy, maurizio.grasselli@polimi.it

${ }^{\ddagger}$ School of Mathematical Sciences and Shanghai Key Laboratory for Contemporary Applied Mathematics, Fudan University, Shanghai 200433, China, haowufd@fudan.edu.cn, haowufd@yahoo.com
} 
diffuse-interface method, such phenomena occurring in a fluid mixture confined in a HeleShaw cell are described by the so-called Cahn-Hilliard-Hele-Shaw system. This model has been proposed in [25] as a simplification of the well-known "model H" in which the NavierStokes system is coupled with the convective Cahn-Hilliard equation (see, e.g., [22]). In the Boussinesq limit, taking the density to be a constant (the case of matched densities) and neglecting gravity, the resulting system takes the following form

$$
\left\{\begin{array}{l}
\boldsymbol{u}=-\nabla P+\mu \nabla \varphi, \\
\operatorname{div} \boldsymbol{u}=0, \\
\varphi_{t}+\boldsymbol{u} \cdot \nabla \varphi=\Delta \mu, \\
\mu=-\Delta \varphi+\Psi^{\prime}(\varphi),
\end{array} \quad \text { in } \Omega \times(0, T) .\right.
$$

Throughout this paper, we assume that $\Omega \subset \mathbb{R}^{d}, d=2,3$, is a bounded domain with smooth boundary $\partial \Omega$. System (1.1) is usually subject to the following natural boundary and initial conditions

$$
\left\{\begin{array}{l}
\boldsymbol{u} \cdot \mathbf{n}=\partial_{\mathbf{n}} \mu=\partial_{\mathbf{n}} \varphi=0, \\
\varphi(\cdot, 0)=\varphi_{0}, \quad \text { in } \Omega .
\end{array}\right.
$$

where $\mathbf{n}$ is the unit outward normal vector to the boundary $\partial \Omega$. In (1.1), $\boldsymbol{u}$ is the averaged fluid velocity, $P$ is the pressure, the order parameter (or phase field) $\varphi$ stands for the relative concentration difference (i.e., the difference in volume fractions) and $\mu$ is the chemical potential for $\varphi$. Physical parameters in (1.1) have been scaled to be one, since they do not play a role in the subsequent analysis.

The total free energy of system (1.1) is of Ginzburg-Landau type

$$
\mathcal{E}(\varphi)=\int_{\Omega}\left(\frac{1}{2}|\nabla \varphi|^{2}+\Psi(\varphi)\right) \mathrm{d} x
$$

which accounts for interfacial energy and unmixing tendencies of the binary mixture. The potential function $\Psi$ is given by (see [7])

$$
\Psi(s)=\frac{\Theta}{2}[(1+s) \log (1+s)+(1-s) \log (1-s)]-\frac{\Theta_{0}}{2} s^{2}, \quad s \in[-1,1],
$$

in which $\Theta$ denotes the absolute temperature of the mixture, while $\Theta_{0}$ is the critical temperature such that if $0<\Theta<\Theta_{0}$ then the phase separation process takes place. We recall that, in the literature, the potential stated in (1.4) whose first derivative $\Psi^{\prime}(s)$ is singular at \pm 1 , is very often approximated by a fourth-order polynomial, namely

$$
\Psi_{0}(s)=\frac{\kappa}{4}\left(s^{2}-1\right)^{2}, \quad s \in \mathbb{R},
$$

where $\kappa>0$ is a constant related to $\Theta_{0}$. This regular approximation is justified whenever $\Theta$ is close to $\Theta_{0}$. Nonetheless, if $\Psi$ is replaced with the regular potential $\Psi_{0}$ then it is impossible to ensure that $\varphi$ takes value in the physically admissible interval $[-1,1]$, since the fourth-order Cahn-Hilliard equation does not enjoy a maximum principle.

In spite of its apparent simplicity, system (1.1) is quite challenging from the theoretical point of view. Most of the available papers are rather recent and only treat the regular 
potential case (1.5). In the periodic setting, existence and uniqueness of global classical solutions in dimension two and the existence of local strong solutions along with certain blow-up criteria in dimension three were proven in [35]. In the same setting, the long-time behavior of global solutions and the stability of local energy minimizers in both two and three dimensions were analyzed in [34]. System (1.1) endowed with boundary conditions (1.2) in a special domain (a box or a rectangle) was considered in [26], where existence, uniqueness and regularity of global two-dimensional (or local three-dimensional) strong solutions were established. In addition, the authors proved the (local) exponential stability of a constant state (i.e., the average of total mass for the initial datum) outside the spinodal region under suitable smallness assumptions either on the initial data or the domain size. The sharp-interface limit of (1.1) and its variant was investigated quite recently in [13,27]. Besides, relationship between the original system (1.1) and the Cahn-Hilliard-Brinkman model (namely, with a regularizing term $-\Delta \boldsymbol{u}$ on the left-hand side of the first equation in (1.1)) was investigated in [5]. We also refer to [12] for nonlocal versions of CahnHilliard-Brinkman/Cahn-Hilliard-Hele-Shaw models with regular potential (1.5) and to [9] for the Cahn-Hilliard-Brinkman model with singular potential (1.4) and nonconstant viscosity. It is also worth mentioning that, in the recent paper [23], the authors analyze a variant of (1.1) with regular potential (1.5) and $\operatorname{div} \boldsymbol{u}=S$, where $S$ is a given space-time dependent mass source that also appears as a forcing term in the Cahn-Hilliard equation. The existence of global weak solutions and local strong solutions is proven. In addition, the authors investigate the long-time behavior in dimension two. More precisely, they demonstrate the existence of a pullback attractor and the convergence of any global weak solution to a single equilibrium as $t \rightarrow+\infty$. Another recent contribution is concerned with the case of unmatched densities (see [11]). The authors demonstrate the existence of a weak solution, discuss the sharp interface limit and present several numerical computations. Regarding the latter issue see also $[14,36]$.

The goal of this paper is to provide a fairly complete analysis of the initial boundary value problem (1.1)-(1.2) when $\Psi$ is singular (e.g., $\Psi$ satisfies the physically relevant case (1.4)). First, we establish the existence of a global weak solution with finite energy in both two and three dimensions (see Theorem 2.1). Then we show that any finite energy solution satisfies a dissipative energy inequality (see Theorem 2.2), which provides a base to study the long-time behavior of the system.

In two dimensions, further results can be obtained. First of all, we demonstrate the uniqueness (up to a constant) of global weak solutions $(\boldsymbol{u}, P, \varphi)$ with finite energy along with a continuous dependence estimate (see Theorem 2.3). This result is somewhat surprising, since the same problem remains an open issue for the case with regular potentials like (1.5) (see, e.g., [23]). The goal can be achieved mainly due to the singular nature of the potential $\Psi$, which yields a $L^{\infty}$-estimate on $\varphi$ as well as the additional regularity $\varphi \in L^{4}\left(0, T ; H^{2}(\Omega)\right)$ within the class of global weak solutions. Then we show that every global weak solution regularizes in finite time and, in particular, we prove the validity of the so-called strict separation property for $\varphi$ (see Theorem 2.4). This means that any global weak solution stays instantaneously away from the pure states \pm 1 provided that it is not a pure state at $t=0$, which is ensured by the assumption $|\Omega|^{-1} \int_{\Omega} \varphi_{0} \mathrm{~d} x \in(-1,1)$ (see also $[9,17,19,28]$ for other models). In particular, the obtained estimates are uni- 
form with respect to bounds on the initial energy and the spatial average. (see Theorem 2.4). Moreover, a similar argument easily yields the existence of a unique global strong solution to (1.1)-(1.2) for arbitrary regular initial datum $\varphi_{0}$. Low regularity of the fluid velocity $\boldsymbol{u}$ that satisfies a Darcy's equation brings us several extra difficulties in the proof. This complexity seems a hard task to overcome in three dimensions (cf. [23, 26, 34, 35]) unless some restrictions are imposed. For instance, we are able to show that if the initial datum is regular and sufficiently close to a local energy minimizer of the total energy $\mathcal{E}$ (see (1.3)), then the system admits a unique (up to a constant) global strong solution $(\boldsymbol{u}, P, \varphi)$ such that $\varphi$ stays in a small neighborhood of that minimizer for all $t \geq 0$ (see Theorem 2.5). This immediately yields a local Lyapunov stability property for any local energy minimizer of $\mathcal{E}$. One of the main difficulties in the proof is that we require $\varphi$ to be strictly separate from the pure state \pm 1 uniformly in time. This issue is nontrivial due to the loss of maximum principle for Cahn-Hilliard type equations and the argument for the two dimensional case in Theorem 2.4 does not apply. Combining the Lojasiewicz-Simon approach (see, e.g., [32]) with suitable interpolation inequalities, we overcome this obstacle and achieve our goal near local minimizers of $\mathcal{E}$, where the "drop" of total energy is ensured to be small during the whole evolution.

The convergence to a single equilibrium of global solutions to Cahn-Hilliard equation is nontrivial, since the topology of the set of equilibria can be rather complicated in high dimensional case and it may form a continuum (see e.g., [31]). Employing a ŁojasiewiczSimon type inequality (see Lemma 6.2), we prove that under suitable assumption on the singular potential (i.e., the real analyticity in $(-1,1)$ ), any global strong solution to problem (1.1)-(1.2) does converge to a single equilibrium as $t \rightarrow+\infty$ (see Theorem 2.6). In dimension two, this is also true for any global weak solution, owing to the strong separation property given in Theorem 2.4. Finally, we give a result on the exponential stability of the averaged initial mass $\bar{\varphi}_{0}$, provided that this constant state is assumed to be outside the spinodal region for $\Psi$ (see Theorem 6.2). This follows from the same argument devised in [26] for a regular potential (1.5).

The plan of this paper goes as follows. In the next section, we introduce function spaces and interpolation inequalities that will be used in our subsequent analysis, as well as a summary of our main mathematical results. In Section 3, we establish the existence of global weak solutions in two and three dimensions. Section 4 is devoted to the uniqueness of weak solutions in dimension two, while high-order regularity and the strict separation property are proved in Section 5. In the final Section 6, we first prove the existence of a unique global strong solution near any local energy minimizer as well as its Lyapunov stability in dimension three. Then we show the convergence to a single equilibrium for any bounded regular solution as $t \rightarrow+\infty$. This result applies to the strong solutions close to the local minimizers but, in two dimensions, it applies to any weak solution. Some useful lemmas on an elliptic equation with a monotone singular term are reported in Appendix. 


\section{Mathematical Setting and Main Results}

\subsection{Notation and function spaces}

Let $X$ be a (real) Banach or Hilbert space, whose norm is denoted by $\|\cdot\|_{X} \cdot X^{\prime}$ indicates the dual space of $X$ and $\langle\cdot, \cdot\rangle$ denotes the corresponding duality product. The boldface letter $\mathbf{X}$ stands for the vectorial space $X^{d}$ endowed with the product structure ( $d$ is the spatial dimension). We denote by $L^{p}(\Omega)$ and $W^{k, p}(\Omega), k \in \mathbb{N}$ and $p \in[1,+\infty]$, the usual Lebesgue spaces and Sobolev spaces of real measurable functions on the domain $\Omega$. We indicate by $H^{k}(\Omega)$ the Hilbert spaces $W^{k, 2}(\Omega)$ with respect to the scalar product $\langle u, v\rangle_{k}=\sum_{|\zeta| \leq k} \int_{\Omega} D^{\zeta} u(x) D^{\zeta} v(x) \mathrm{d} x\left(\zeta=\left(\zeta_{1}, \ldots, \zeta_{d}\right)\right.$ being a multi-index $)$ and the induced norm $\|u\|_{H^{k}(\Omega)}=\sqrt{\langle u, u\rangle_{k}}$. Given an interval $I$ of $\mathbb{R}^{+}$, we introduce the function space $L^{p}(I ; X)$ with $p \in[1,+\infty]$, which consists of Bochner measurable $p$-integrable functions with values in the Banach space $X$.

We set $H=L^{2}(\Omega)$. Its inner product and the associate norm are denoted by $(\cdot, \cdot)$ and $\|\cdot\|$, respectively. Then we set $V=H^{1}(\Omega)$ endowed with the norm $\|f\|_{V}^{2}=\|\nabla f\|^{2}+\|f\|^{2}$. For every $f \in V^{\prime}=\left(H^{1}(\Omega)\right)^{\prime}$, we denote by $\bar{f}$ the average of function $f$ over $\Omega$ such that

$$
\bar{f}=\frac{1}{|\Omega|}\langle f, 1\rangle \text {. }
$$

Then we recall the following Poincaré's inequality

$$
\|f-\bar{f}\| \leq C_{P}\|\nabla f\|, \quad \forall f \in V,
$$

where $C_{P}$ is a constant depending only on $d$ and $\Omega$. We introduce the linear spaces

$$
V_{0}=\{u \in V: \bar{u}=0\}, \quad L_{0}^{2}(\Omega)=\{u \in H: \bar{u}=0\}, \quad V_{0}^{\prime}=\left\{u \in V^{\prime}: \bar{u}=0\right\},
$$

and we consider the linear operator $A \in \mathcal{L}\left(V, V^{\prime}\right)$ defined by

$$
\langle A u, v\rangle=\int_{\Omega} \nabla u \cdot \nabla v \mathrm{~d} x, \quad \forall u, v \in V .
$$

The restriction of $A$ from $V_{0}$ onto $V_{0}^{\prime}$ is an isomorphism. In particular, $A$ is positively defined on $V_{0}$ and self-adjoint. We denote its inverse map by $\mathcal{N}=A^{-1}: V_{0}^{\prime} \rightarrow V_{0}$. Note that for every $f \in V_{0}^{\prime}, u=\mathcal{N} f \in V$ is the unique weak solution of the Neumann problem

$$
\begin{cases}-\Delta u=f, & \text { in } \Omega, \\ \partial_{\mathbf{n}} u=0, & \text { on } \partial \Omega .\end{cases}
$$

Besides, we have

$$
\begin{aligned}
& \langle A u, \mathcal{N} f\rangle=\langle u, f\rangle, \quad \forall u \in V, \forall f \in V_{0}^{\prime}, \\
& \langle f, \mathcal{N} g\rangle=\langle\mathcal{N} f, g\rangle=\int_{\Omega} \nabla(\mathcal{N} f) \cdot \nabla(\mathcal{N} g) \mathrm{d} x, \quad \forall f, g \in V_{0}^{\prime} .
\end{aligned}
$$

For any $f \in V_{0}^{\prime}$, we set $\|f\|_{V_{0}^{\prime}}=\|\nabla \mathcal{N} f\|$. It is well-known that $f \rightarrow\|f\|_{V_{0}^{\prime}}$ and $f \rightarrow$ $\left(\|f-\bar{f}\|_{V_{0}^{\prime}}^{2}+|\bar{f}|^{2}\right)^{\frac{1}{2}}$ are equivalent norms on $V_{0}^{\prime}$ and $V^{\prime}$, respectively. Besides, according 
to Poincaré's inequality (2.1), we have that $f \rightarrow\left(\|\nabla f\|^{2}+|\bar{f}|^{2}\right)^{\frac{1}{2}}$ is an equivalent norm on $V$. We also report the following standard Hilbert interpolation inequality and elliptic estimates for the Neumann problem

$$
\begin{aligned}
\|f\| & \leq\|f\|_{V_{0}^{\prime}}^{\frac{1}{2}}\|\nabla f\|^{\frac{1}{2}}, & & \forall f \in V_{0}, \\
\|\nabla \mathcal{N} f\|_{\mathbf{H}^{k}(\Omega)} & \leq C\|f\|_{H^{k-1}(\Omega)}, & & \forall f \in H^{k-1}(\Omega) \cap L_{0}^{2}(\Omega), \quad k=1,2 .
\end{aligned}
$$

Next, for the velocity vector field $\boldsymbol{u}$, we introduce the solenoidal Hilbert space

$$
\mathbf{H}_{\sigma}=\left\{\boldsymbol{u} \in \mathbf{L}^{2}(\Omega): \operatorname{div} \boldsymbol{u}=0 \text { in } \Omega, \boldsymbol{u} \cdot \mathbf{n}=0 \text { on } \partial \Omega\right\}
$$

endowed with the usual norm $\|\cdot\|_{\mathbf{L}^{2}(\Omega)}$. Let $\Pi$ be the orthogonal Leray projection in $\mathbf{H}=\mathbf{L}^{2}(\Omega)$ on $\mathbf{H}_{\sigma}$. It is well known that every vector field $\boldsymbol{u} \in \mathbf{H}$ can be uniquely represented as

$$
\boldsymbol{u}=\boldsymbol{v}+\nabla P
$$

where $\boldsymbol{v}=\Pi \boldsymbol{u} \in \mathbf{H}_{\sigma}$ and $P \in V_{0}$. We recall that $\Pi$ is a bounded operator from $\mathbf{W}^{k, p}(\Omega)$ $(1<p<\infty, k \geq 0)$ into itself (cf. [18, Lemma 3.3]), namely

$$
\|\Pi \boldsymbol{u}\|_{\mathbf{W}^{k, p}} \leq C\|\boldsymbol{u}\|_{\mathbf{W}^{k, p}}, \quad \forall \boldsymbol{u} \in \mathbf{W}^{k, p}(\Omega),
$$

where the constant $C$ is independent of $\boldsymbol{u}$. On the other hand, if $\boldsymbol{u} \in \mathbf{W}^{k, p}(\Omega)$, with $1<p<\infty$, then from $\nabla P=\boldsymbol{u}-\Pi \boldsymbol{u}$ we see that $P$ can be the unique solution of the Neumann problem

$$
\begin{cases}-\Delta P=\operatorname{div} \boldsymbol{u}, & \text { in } \Omega, \\ \partial_{\mathbf{n}} P=\boldsymbol{u} \cdot \mathbf{n}, & \text { on } \partial \Omega,\end{cases}
$$

satisfying $\bar{P}=0$. Hence, it follows that $P \in W^{k+1, p}(\Omega)$ by the classical elliptic regularity results for the Neumann problem. In addition, solenoidal vector fields in $\mathbf{V}=\mathbf{H}^{1}(\Omega)$ satisfy the following inequality (see e.g., [20, Theorem 3.8])

$$
\|\boldsymbol{v}\|_{\mathbf{v}} \leq C(\|\nabla \times \boldsymbol{v}\|+\|\boldsymbol{v}\|), \quad \forall \boldsymbol{v} \in \mathbf{V} \cap \mathbf{H}_{\sigma},
$$

for some constant $C$ independent of $\boldsymbol{u}$. Below we will also use the higher-order soleinodal Hilbert space

$$
\mathbf{V}_{\sigma}=\{\boldsymbol{u} \in \mathbf{V}: \operatorname{div} \boldsymbol{u}=0 \text { in } \Omega, \quad \boldsymbol{u}=0 \text { on } \partial \Omega\}
$$

equipped with inner product and norm

$$
\langle\boldsymbol{u}, \boldsymbol{v}\rangle_{\mathbf{V}_{\sigma}}=(\nabla \boldsymbol{u}, \nabla \boldsymbol{v}), \quad\|\boldsymbol{u}\|_{\mathbf{V}_{\sigma}}=\|\nabla \boldsymbol{u}\|
$$

For the reader's convenience, we recall here some well-known interpolation inequalities in Sobolev spaces which can be found in classical literature (see e.g. [6,33]):

$\diamond$ Ladyzhenskaya's inequality

$$
\|f\|_{L^{4}(\Omega)} \leq C\|f\|^{\frac{1}{2}}\|f\|_{V}^{\frac{1}{2}}, \quad \forall f \in V, \text { if } d=2 .
$$


$\diamond$ Agmon's inequality

$$
\begin{aligned}
& \|f\|_{L^{\infty}(\Omega)} \leq C\|f\|^{\frac{1}{2}}\|f\|_{H^{2}(\Omega)}^{\frac{1}{2}}, \quad \forall f \in H^{2}(\Omega), \text { if } d=2, \\
& \|f\|_{L^{\infty}(\Omega)} \leq C\|f\|_{V}^{\frac{1}{2}}\|f\|_{H^{2}(\Omega)}^{\frac{1}{2}}, \quad \forall f \in H^{2}(\Omega), \text { if } d=3 .
\end{aligned}
$$

$\diamond$ Gagliardo-Nirenberg inequality

$$
\left\|D^{j} f\right\|_{L^{p}(\Omega)} \leq C\|f\|_{L^{q}(\Omega)}^{1-a}\|f\|_{W^{m, r}(\Omega)}^{a}, \quad \forall f \in W^{m, r}(\Omega) \cap L^{q}(\Omega),
$$

where $j, m$ are arbitrary integers satisfying $0 \leq j<m$ and $\frac{j}{m} \leq a \leq 1$, and $1 \leq$ $q, r \leq+\infty$ such that

$$
\frac{1}{p}-\frac{j}{d}=a\left(\frac{1}{r}-\frac{m}{d}\right)+(1-a) \frac{1}{q} .
$$

If $1<r<+\infty$ and $m-j-\frac{n}{r}$ is a nonnegative integer, then the above inequality holds only for $\frac{j}{m} \leq a<1$.

Also, we recall the following standard results on the differentiation of a product in Sobolev spaces $(d=2,3)$ :

$$
\begin{gathered}
\|f g\|_{V} \leq C\left(\|f\|_{V}\|g\|_{L^{\infty}(\Omega)}+\|f\|_{L^{\infty}(\Omega)}\|g\|_{V}\right), \quad \forall f, g \in V \cap L^{\infty}(\Omega), \\
\|f g\|_{V} \leq C\left(\|f\|_{W^{1,4}(\Omega)}\|g\|_{L^{4}(\Omega)}+\|f\|_{L^{\infty}(\Omega)}\|g\|_{V}\right), \quad \forall f \in W^{1,4}(\Omega), g \in V, \\
\|f g\|_{H^{2}(\Omega)} \leq C\left(\|f\|_{H^{2}(\Omega)}\|g\|_{L^{\infty}(\Omega)}+\|f\|_{L^{\infty}(\Omega)}\|g\|_{H^{2}(\Omega)}\right), \quad \forall f, g \in H^{2}(\Omega) .
\end{gathered}
$$

Throughout the paper, if it is not otherwise stated, we indicate by $C$ a generic positive constant depending only on the domain and on structural quantities. The constant $C$ may vary from line to line and even within the same line. Any further dependence will be explicitly pointed out if necessary.

\subsection{Main results}

Our assumptions on the singular potential $\Psi$ are the following:

(H) $\Psi$ can be decomposed into the form

$$
\Psi(s)=S(s)-\frac{\Theta_{0}}{2} s^{2}, \quad \forall s \in[-1,1],
$$

where the function $S:[-1,1] \mapsto \mathbb{R}$ satisfies $S \in \mathcal{C}([-1,1]) \cap \mathcal{C}^{2}(-1,1)$,

$$
\begin{array}{r}
\lim _{s \rightarrow-1^{+}} S^{\prime}(s)=-\infty, \quad \lim _{s \rightarrow 1^{-}} S^{\prime}(s)=+\infty, \\
S^{\prime \prime}(s) \geq \Theta>0, \quad \forall s \in(-1,1),
\end{array}
$$

with the constants $\Theta_{0}, \Theta$ satisfying

$$
\Theta_{0}-\Theta:=\alpha>0 \text {. }
$$

In addition, there exists $\kappa \in(0,1)$ such that $S^{\prime \prime}$ is non-decreasing in $[1-\kappa, 1)$ and nonincreasing in $(-1,-1+\kappa]$. Without loss of generality, we assume $S(0)=S^{\prime}(0)=0$. Finally, we make the extension that

$$
S(s)=+\infty, \text { for all }|s|>1 .
$$


Remark 2.1. Assumption (H) is satisfied in the case of the logarithmic double-well potential (1.4) with

$$
S(s)=\frac{\Theta}{2}[(1+s) \log (1+s)+(1-s) \log (1-s)], \quad \forall s \in[-1,1] .
$$

The case $\Theta_{0}-\Theta \leq 0$ is easier, since in this case the potential $\Psi$ is already convex and we can simply consider $\Psi$ without the decomposition (2.15).

Next, we introduce the notion of finite energy weak solution to the initial boundary value problem (1.1)-(1.2).

Definition 2.1. Let $\varphi_{0} \in V$ be such that $S\left(\varphi_{0}\right) \in L^{1}(\Omega)$ and $\bar{\varphi}_{0} \in(-1,1)$. For $T>0$, a triple $(\boldsymbol{u}, P, \varphi)$ is a weak solution with finite energy to problem (1.1)-(1.2) on the time interval $[0, T]$, if

$$
\begin{aligned}
& \boldsymbol{u} \in L^{2}\left(0, T ; \mathbf{H}_{\sigma}\right), \quad P \in L^{\frac{8}{5}}\left(0, T ; V_{0}\right), \\
& \varphi \in L^{\infty}(0, T ; V) \cap L^{4}\left(0, T ; H^{2}(\Omega)\right) \cap H^{1}\left(0, T ; V^{\prime}\right), \\
& \varphi \in L^{\infty}(\Omega \times(0, T)) \text { with }|\varphi(x, t)|<1 \text { a.e. }(x, t) \in \Omega \times(0, T), \\
& \Psi(\varphi) \in L^{\infty}\left(0, T ; L^{1}(\Omega)\right), \quad \Psi^{\prime}(\varphi) \in L^{2}(0, T ; H), \\
& \mu \in L^{2}(0, T ; V),
\end{aligned}
$$

and

$$
\left\langle\varphi_{t}, v\right\rangle+(\boldsymbol{u} \cdot \nabla \varphi, v)+(\nabla \mu, \nabla v)=0, \quad \forall v \in V, \text { a.e. } t \in(0, T)
$$

where

$$
\mu=-\Delta \varphi+\Psi^{\prime}(\varphi), \quad \boldsymbol{u}=-\nabla P+\mu \nabla \varphi \quad \text { a.e. }(x, t) \in \Omega \times(0, T) .
$$

Moreover, $\partial_{n} \varphi=0$ and $\varphi(0, \cdot)=\varphi_{0}$ almost everywhere on $\partial \Omega \times(0, T)$ and in $\Omega$, respectively.

Remark 2.2. According to the Darcy's equation for $\boldsymbol{u}$, the above Definition 2.1 for weak solutions is equivalent to the following weak formulation

$$
\begin{aligned}
& \int_{\Omega} \boldsymbol{u} \cdot \boldsymbol{v} \mathrm{d} x=\int_{\Omega} \mu \nabla \varphi \cdot \boldsymbol{v} \mathrm{d} x \\
& \int_{\Omega} \nabla P \cdot \nabla \psi \mathrm{d} x=\int_{\Omega} \mu \nabla \varphi \cdot \nabla \psi \mathrm{d} x,
\end{aligned}
$$

for almost every $t \in(0, T)$ and for any $\boldsymbol{v} \in \mathbf{H}_{\sigma}, \psi \in H^{1}(\Omega)$. Thus, the pressure $P$ is recovered by the second equation. In addition, in light of the boundary conditions and the identity

$$
\mu \nabla \varphi=\nabla\left(\frac{1}{2}|\nabla \varphi|^{2}+\Psi(\varphi)\right)-\operatorname{div}(\nabla \varphi \otimes \nabla \varphi),
$$

we can rewrite the weak formulation of Darcy's equation as

$$
\int_{\Omega} \boldsymbol{u} \cdot \boldsymbol{v} \mathrm{d} x=\int_{\Omega} \varphi \nabla \mu \cdot \boldsymbol{v} \mathrm{d} x=-\int_{\Omega} \operatorname{div}(\nabla \varphi \otimes \nabla \varphi) \cdot \boldsymbol{v} \mathrm{d} x
$$

We can now state the main results of this paper. The first result concerns the existence of global weak solutions, in both two and three dimensions. 
Theorem 2.1 (Global weak solutions with finite energy). Let $d=2,3$. Assume that $(H)$ is satisfied and $\varphi_{0} \in V$ with $S\left(\varphi_{0}\right) \in L^{1}(\Omega)$ and $\left|\bar{\varphi}_{0}\right|<1$. Then, for any $T>0$, there exists at least one global weak solution with finite energy $(\boldsymbol{u}, P, \varphi)$ to problem $(1.1)-(1.2)$ on $[0, T]$ in the sense of Definition 2.1 such that, in addition,

$$
\begin{aligned}
& \boldsymbol{u} \in L^{s}(0, T ; \mathbf{V}), \quad P \in L^{q}\left(0, T ; H^{2}(\Omega)\right) \\
& \varphi \in \mathcal{C}([0, T], V) \cap L^{2}\left(0, T ; W^{2, p}(\Omega)\right) \\
& \Psi^{\prime}(\varphi) \in L^{2}\left(0, T ; L^{p}(\Omega)\right)
\end{aligned}
$$

where $s=\frac{6}{5}$ if $d=3$ or $s \in\left[1, \frac{4}{3}\right)$ if $d=2 ; q=\frac{8}{7}$ is $d=3$ or $1 \leq q<\frac{6}{5}$ if $d=2 ; p=6$ if $d=3$ or $2 \leq p<\infty$ if $d=2$. Moreover, every global weak solution satisfies the energy identity

$$
\frac{\mathrm{d}}{\mathrm{d} t} \mathcal{E}(\varphi(t))+\|\boldsymbol{u}(t)\|^{2}+\|\nabla \mu(t)\|^{2}=0, \quad \text { for a.e. } t \in(0, T)
$$

as well as the mass conservation

$$
\int_{\Omega} \varphi(t) \mathrm{d} x=\int_{\Omega} \varphi_{0} \mathrm{~d} x, \quad \forall t \in[0, T] .
$$

Remark 2.3. Assumption $\left|\bar{\varphi}_{0}\right|<1$ indicates that the initial datum is not allowed to be a pure state (i.e. \pm 1$)$. On the other hand, we observe that if the initial datum is a pure state then no separation process will take place because we now have a single fluid. We recall that a similar result was obtained as a by-product in [10] by assuming a homogeneous Dirichlet boundary condition for $\mu$.

Next, we show that any global weak solution is dissipative, namely,

Theorem 2.2 (Dissipativity). Let the assumptions of Theorem 2.1 hold. Then, any global weak solution with finite energy $(\boldsymbol{u}, P, \varphi)$ satisfies the following dissipative estimate

$$
\mathcal{E}(\varphi(t))+\int_{t}^{t+1}\left(\|\boldsymbol{u}(\tau)\|^{2}+\|\nabla \mu(\tau)\|^{2}\right) \mathrm{d} \tau \leq C \mathcal{E}\left(\varphi_{0}\right) \mathrm{e}^{-\omega t}+C, \quad \forall t \geq 0
$$

where $\omega$ and $C$ are positive constants independent of the initial datum. Moreover, we have for all $t \geq 0$

$$
\int_{t}^{t+1}\left(\|\varphi(\tau)\|_{H^{2}(\Omega)}^{4}+\|\varphi(\tau)\|_{W^{2, p}(\Omega)}^{2}+\left\|S^{\prime}(\varphi(\tau))\right\|_{L^{p}(\Omega)}^{2}\right) \mathrm{d} \tau \leq\left(C \mathcal{E}\left(\varphi_{0}\right) \mathrm{e}^{-\omega t}+C\right)^{2},
$$

where $p=6$ if $d=3$ or $p \in[2,+\infty)$ if $d=2$. Here, the positive constant $C$ depends on $\bar{\varphi}_{0} \in(-1,1)$ and the parameter $p$, but is independent of other norms of the initial datum.

Uniqueness of weak solutions for the Cahn-Hilliard-Hele-Shaw system (1.1) turns out to be a rather hard task, due to the low regularity of the velocity field $\boldsymbol{u}$ (cf. [23,34] for the case of regular potentials, where the uniqueness remains an open question even in two dimensions). However, for a class of free energy densities that contains, in particular, the physical relevant singular potential $\Psi$ in (1.4), we are able to prove the following continuous dependence result on initial data in a lower-order function space (i.e., $V_{0}^{\prime}$ ) when $d=2$. 
Theorem 2.3 (Uniqueness of global finite energy weak solutions in $d=2$ ). Let $d=2$ and $(H)$ be satisfied. Assume that $\varphi_{0 i} \in V$ with $S\left(\varphi_{0 i}\right) \in L^{1}(\Omega), i=1,2$, and $\bar{\varphi}_{01}=$ $\bar{\varphi}_{02}=m \in(-1,1)$. Then, any pair of global weak solutions $\left(\boldsymbol{u}_{1}, P_{1}, \varphi_{1}\right)$ and $\left(\boldsymbol{u}_{2}, P_{2}, \varphi_{2}\right)$ to problem (1.1)-(1.2) on $[0, T]$ with initial data $\varphi_{01}$ and $\varphi_{02}$, respectively, fulfills the following estimate

$$
\left\|\varphi_{1}(t)-\varphi_{2}(t)\right\|_{V_{0}^{\prime}}^{2}+\int_{0}^{t}\left\|\varphi_{1}(\tau)-\varphi_{2}(\tau)\right\|_{V}^{2} \mathrm{~d} \tau \leq C\left\|\varphi_{01}-\varphi_{02}\right\|_{V_{0}^{\prime}}^{2}
$$

for every $t \in[0, T]$. Here, the positive constant $C$ depends on $T$ as well as on the initial energy $\mathcal{E}\left(\varphi_{0 i}\right), i=1,2$. In particular, the global weak solution with finite energy to problem (1.1)-(1.2) is unique.

The uniqueness of weak solutions is strictly connected with their regularity properties. Indeed, we can show that global weak solutions with finite energy become more regular instantaneously for $t>0$ when the spatial space dimension is two. Furthermore, we are able to prove the validity of the strict separation property on the time interval $[\sigma, \infty)$ with arbitrary $\sigma>0$. More precisely, we have

Theorem 2.4 (Regularity of finite energy weak solutions in $d=2$ ). Let $d=2$ and (H) be satisfied. Assume that the initial datum $\varphi_{0}$ has finite energy $\mathcal{E}\left(\varphi_{0}\right) \leq R$ for some $R>0$ and $\bar{\varphi}_{0}=m \in(-1,1)$. Then, for every $\sigma>0$ and $p \geq 1$, there exists a positive constant $C=C(\sigma, p, m, R)$ such that

$$
\|\boldsymbol{u}\|_{L^{\infty}(\sigma, \infty ; \mathbf{V})}+\|P\|_{L^{\infty}\left(\sigma, \infty ; H^{2}(\Omega)\right)}+\|\varphi\|_{L^{\infty}\left(\sigma, \infty ; W^{2, p}(\Omega)\right)} \leq C .
$$

In addition, suppose that the second derivative $S^{\prime \prime}$ is convex and satisfies the following inequality

$$
\left|S^{\prime \prime}(s)\right| \leq e^{C\left|S^{\prime}(s)\right|+C}, \quad \forall s \in(-1,1),
$$

for some positive constant $C$. Then, for every $\sigma>0$, there exists $\delta=\delta(\sigma, m, R) \in(0,1)$ such that

$$
\|\varphi(t)\|_{L^{\infty}(\Omega)} \leq 1-\delta, \quad \forall t \geq 2 \sigma
$$

and

$$
\|\boldsymbol{u}\|_{L^{\infty}\left(2 \sigma, t ; \mathbf{H}^{2}(\Omega)\right)}+\|P\|_{L^{\infty}\left(2 \sigma, t ; H^{3}(\Omega)\right)}+\|\varphi\|_{L^{\infty}\left(2 \sigma, t ; H^{4}(\Omega)\right)} \leq C, \quad \forall t \geq 2 \sigma,
$$

where $C=C(\sigma, t, m, R)>0$.

Remark 2.4. It is easy to verify that the additional assumption (2.23) is satisfied in the case of the logarithmic potential $S$ given by (2.16).

Theorem 2.4 easily implies the existence of a unique global strong solution to problem (1.1)-(1.2) in two dimensions, provided that the initial datum $\varphi_{0}$ is more regular, e.g., $\mu(0)=-\Delta \varphi_{0}+\Psi^{\prime}\left(\varphi_{0}\right) \in V$ with $\partial_{\mathbf{n}} \varphi_{0}=0$ on $\partial \Omega$. However, when the spatial dimensional is three, the existence of a unique global strong solution with an arbitrary large regular initial datum $\varphi_{0}$ is not expected (cf. [34] for the case with regularity potential). Nevertheless, if the initial datum is regular enough and sufficiently close to any local energy minimizer of the total energy $\mathcal{E}$, we are able to prove the existence of a unique solution within a suitable regularity class. To this end, we recall the following definition 
Definition 2.2. Set

$$
\mathcal{Z}_{m}=\left\{\varphi \in V:\|\varphi\|_{L^{\infty}(\Omega)} \leq 1, \bar{\varphi}=m \in(-1,1)\right\} .
$$

Any $\psi \in \mathcal{Z}_{m}$ is called a local energy minimizer of the total energy $\mathcal{E}$ defined in (1.3), if there exists a constant $\chi>0$ such that $\mathcal{E}(\psi) \leq \mathcal{E}(\varphi)$ for all $\varphi \in \mathcal{Z}_{m}$ satisfying $\|\varphi-\psi\|_{V}<\chi$. If $\chi=+\infty$, then $\psi$ is called a global energy minimizer of $\mathcal{E}$.

Besides, the following additional assumptions on the function $S$ will be necessary for global strong well-posedness in three dimensions, which are still valid for the logarithmic potential given by $(2.16)$ :

(H1) $S \in \mathcal{C}([-1,1]) \cap \mathcal{C}^{4}(-1,1)$ and there exists $\kappa \in(0,1)$ such that

$$
S^{(3)}(s) s \geq 0 \quad \text { and } \quad S^{(4)}(s)>0, \quad \forall s \in(-1,-1+\kappa] \cup[1-\kappa, 1) .
$$

Then we can prove

Theorem 2.5 (Global strong solution and Lyapunov stability in $d=3$ ). Let $d=3$. Suppose that the assumptions (H) and (H1) hold. In addition, assume that $S$ is real analytic in $(-1,1)$ and $\psi \in \mathcal{Z}_{m}$ is a local energy minimizer of the total energy $\mathcal{E}$. Then, for any $\epsilon>0$, there exists a constant $\eta \in(0,1)$ such that for an arbitrary initial datum $\varphi_{0} \in H^{3}(\Omega)$ satisfying $\partial_{\mathbf{n}} \varphi_{0}=0$ on $\partial \Omega, \bar{\varphi}_{0}=\bar{\psi}=m$ and $\left\|\varphi_{0}-\psi\right\|_{H^{2}} \leq \eta$, problem (1.1)-(1.2) admits a unique global strong solution $(\boldsymbol{u}, P, \varphi)$ such that

$$
\begin{aligned}
& \boldsymbol{u} \in \mathcal{C}\left([0,+\infty), \mathbf{H}_{\sigma}\right) \cap L_{l o c}^{2}\left(0,+\infty ; \mathbf{H}^{3}(\Omega)\right), \\
& P \in \mathcal{C}([0,+\infty), V) \cap L_{l o c}^{2}\left(0,+\infty ; H^{4}(\Omega)\right), \\
& \varphi \in \mathcal{C}\left([0,+\infty), H^{3}(\Omega)\right) \cap H_{l o c}^{1}(0,+\infty ; V) \cap L_{l o c}^{2}\left(0,+\infty ; H^{5}(\Omega)\right), \\
& \mu \in \mathcal{C}([0,+\infty), V) \cap H_{l o c}^{1}\left(0,+\infty ; V^{\prime}\right) \cap L_{l o c}^{2}\left(0,+\infty ; H^{3}(\Omega)\right) .
\end{aligned}
$$

Moreover, the phase field $\varphi$ always stays close to the minimizer $\psi$ such that

$$
\|\varphi(t)-\psi\|_{H^{2}(\Omega)} \leq \epsilon, \quad \forall t \geq 0 .
$$

Namely, any local energy minimizer of $\mathcal{E}$ is locally Lyapunov stable.

Remark 2.5. The conclusions of Theorem 2.5 (in particular, the Lyapunov stability for local energy minimizers) are still valid in two dimensions, with only minor modifications in the proof mainly due to Sobolev embedding theorems.

Remark 2.6. Actually the solution given by Theorem 2.5 is stronger than the usual notion of strong solution (i.e., a solution which satisfies the equations and the initial and boundary conditions almost everywhere).

Concerning the long-time behavior of problem (1.1)-(1.2), we have 
Theorem 2.6 (Long-time behavior). Assume that $S$ is real analytic in $(-1,1)$. If $d=2$, let $(\boldsymbol{u}, P, \varphi)$ be a global weak solution to problem (1.1)-(1.2) that satisfies Theorem 2.4. If $d=3$, let $(\boldsymbol{u}, P, \varphi)$ be a global strong solution given by Theorem 2.5. Then for both cases, there exists $\varphi_{\infty} \in H^{3}(\Omega)$ which is a strong solution to the stationary Cahn-Hilliard equation

$$
\begin{cases}-\Delta \varphi_{\infty}+\Psi^{\prime}\left(\varphi_{\infty}\right)=\overline{\Psi^{\prime}\left(\varphi_{\infty}\right)}, & \text { in } \Omega \\ \partial_{n} \varphi_{\infty}=0, & \text { on } \partial \Omega \\ \bar{\varphi}_{\infty}=\bar{\varphi}_{0}, & \end{cases}
$$

such that $(\boldsymbol{u}(t), \varphi(t))$ converges to $\left(\mathbf{0}, \varphi_{\infty}\right)$ as $t \rightarrow+\infty$ with the following convergence rate

$$
\|\boldsymbol{u}(t)\|+\left\|\varphi(t)-\varphi_{\infty}\right\|_{H^{3}(\Omega)} \leq C(1+t)^{-\frac{\theta}{1-2 \theta}}, \quad \forall t \geq 1
$$

Here, $C \geq 0$ is a constant depending on $\left\|\varphi_{0}\right\|_{V}($ if $d=2),\left\|\varphi_{0}\right\|_{H^{3}(\Omega)}($ if $d=3),\left\|\varphi_{\infty}\right\|_{H^{3}(\Omega)}$ and $\Psi$, while $\theta \in\left(0, \frac{1}{2}\right)$ is a constant depending only on $\varphi_{\infty}$ (cf. Lemma 6.2).

Remark 2.7. Theorem 2.6 implies that, for any global strong solution $(\boldsymbol{u}, P, \varphi)$ obtained in Theorem 2.5, $\varphi$ will not only stay close to that local energy minimizer $\psi$, but also converge to a certain equilibrium $\varphi_{\infty}$ that is also near $\psi$. Furthermore, if $\psi$ is an isolated minimizer, then it follows that $\varphi_{\infty}=\psi$, namely, $\psi$ is locally asymptotically stable.

\subsection{The infinite dimensional dynamical system}

Before ending this section, we briefly comment on the infinite dimensional dynamical system associated to (1.1)-(1.2). For any $m \in(-1,1)$, consider the phase space $\mathcal{Z}_{m}$ (see (2.26)) with the metric

$$
\mathbf{d}\left(\varphi_{1}, \varphi_{2}\right)=\left\|\nabla\left(\varphi_{1}-\varphi_{2}\right)\right\|
$$

It is well-known that $\mathcal{Z}_{m}$ is a complete metric space. The following result can be proven:

Theorem 2.7 (Generalized semiflow and its global attractor). Let $m \in(-1,1)$. Assume that $(H)$ is satisfied. Denote by $\mathcal{G}_{m}$ the family of all global weak solutions to problem (1.1)(1.2) with initial condition $\varphi_{0} \in \mathcal{Z}_{m}$. Then $\mathcal{G}_{m}$ defines a generalized semiflow on $\mathcal{Z}_{m}$ in the sense of [4] and it admits a unique global attractor.

Thanks to the validity of Theorems 2.1 and 2.2, in particular, the energy identity (2.20) for global weak solutions, the proof of Corollary 2.7 can be carried out by a standard argument (see e.g., [15]) with some minor modifications and thus we leave the details to the interested readers.

Next, when the spatial dimension is two, thanks to the uniqueness result Theorem 2.3 and Theorem 2.4, we have a strongly continuous semigroup acting on the phase space $\mathcal{Z}_{m}$ defined via the rule $\mathbb{S}(t) \varphi_{0}=\varphi(t)$ (see [19][Section 6]). Moreover, Theorem 2.4 also entails that the global attractor is bounded in the more regular space $H^{4}(\Omega)$. Therefore, on account of known results for infinite dimensional dynamical systems, the global attractor obtained in Theorem 2.7 consists of a time-section of complete (i.e., defined on the whole $\mathbb{R}$ ) strong solutions. Exploiting the separation property (2.24), one can proceed to establish the following result through the general approach described in [29]. 
Theorem 2.8 (Exponential attractors in $d=2)$. Let $d=2$ and $m \in(-1,1)$. Assume that $(H)$ and $(2.23)$ are satisfied. The dynamical system $\left(\mathcal{Z}_{m}, \mathbb{S}(t)\right)$ has an exponential attractor that is bounded in $H^{4}(\Omega)$. This further implies, in particular, the global attractor for problem (1.1)-(1.2) has finite fractal dimension.

\section{Global Weak Solutions}

The strategy to prove Theorem 2.1 is based on a standard approximation procedure. First, we introduce a family of regular potentials $\left\{\Psi_{\varepsilon}\right\}$ that suitably approximates the singular potential $\Psi$. Then we establish an existence result to the approximating problem with the regular potential $\Psi_{\varepsilon}$, by means of the Galerkin method. Finally, for the approximate solutions $\left(\boldsymbol{u}_{\varepsilon}, P_{\varepsilon}, \varphi_{\varepsilon}\right)$ related to the family of regular potentials $\left\{\Psi_{\varepsilon}\right\}$, we recover compactness by means of uniform energy estimates with respect to the approximation parameter $\varepsilon$ and we show that as $\varepsilon \rightarrow 0$ the limit triple $(\boldsymbol{u}, P, \varphi)$ is indeed a global weak solution with finite energy to problem (1.1)-(1.2).

\subsection{The approximating problem}

For $\varepsilon \in(0, \kappa)$ with $\kappa$ being the constant given in $(\mathrm{H})$, we introduce a family of regular potentials $\left\{\Psi_{\varepsilon}\right\}$ that approximates the original singular potential $\Psi$ by setting

$$
\Psi_{\varepsilon}(s)=S_{\varepsilon}(s)-\frac{\Theta_{0}}{2} s^{2}, \quad \forall s \in \mathbb{R},
$$

where

$$
S_{\varepsilon}(s)= \begin{cases}\sum_{j=0}^{2} \frac{1}{j !} S^{(j)}(1-\varepsilon)[s-(1-\varepsilon)]^{j}, & \forall s \geq 1-\varepsilon, \\ S(s), & \forall s \in[-1+\varepsilon, 1-\varepsilon], \\ \sum_{j=0}^{2} \frac{1}{j !} S^{(j)}(-1+\varepsilon)[s-(-1+\varepsilon)]^{j}, & \forall s \leq-1+\varepsilon .\end{cases}
$$

By the above construction of $\Psi_{\varepsilon}$, we obtain the following properties that will be useful in the proof of Theorem 2.1.

Lemma 3.1. Assume that $(H)$ is satisfied. Then, there exists $\bar{\kappa} \in(0, \kappa]$ such that for any $\varepsilon \in(0, \bar{\kappa})$, the approximating function $\Psi_{\varepsilon}$ given by (3.1) satisfies

(AH) $\Psi_{\varepsilon} \in \mathcal{C}^{2}(\mathbb{R})$ and

$$
-\widetilde{\alpha} \leq \Psi_{\varepsilon}(s), \quad-\alpha \leq \Psi_{\varepsilon}^{\prime \prime}(s) \leq L, \quad \forall s \in \mathbb{R},
$$

where $\widetilde{\alpha}$ is a positive constant independent of $\varepsilon$, the constant $\alpha$ is given in $(H)$ and $L$ is a positive constant that may depend on $\varepsilon$. 
For every $\varepsilon \in(0, \bar{\kappa})$ and $\Psi_{\varepsilon}$ being the regular potential constructed in (3.1), we consider the approximating problem (AP1):

$$
\left\{\begin{array}{l}
\boldsymbol{u}_{\varepsilon}=-\nabla P_{\varepsilon}+\mu_{\varepsilon} \nabla \varphi_{\varepsilon}, \\
\operatorname{div} \boldsymbol{u}_{\varepsilon}=0, \\
\partial_{t} \varphi_{\varepsilon}+\boldsymbol{u}_{\varepsilon} \cdot \nabla \varphi_{\varepsilon}=\Delta \mu_{\varepsilon}, \\
\mu_{\varepsilon}=-\Delta \varphi_{\varepsilon}+\Psi_{\varepsilon}^{\prime}\left(\varphi_{\varepsilon}\right),
\end{array} \quad \text { in } \Omega \times(0, T),\right.
$$

subject to the initial and boundary conditions

$$
\left\{\begin{array}{l}
\boldsymbol{u}_{\varepsilon} \cdot \mathbf{n}=\partial_{\mathbf{n}} \mu_{\varepsilon}=\partial_{\mathbf{n}} \varphi_{\varepsilon}=0, \quad \text { on } \partial \Omega \times(0, T), \\
\varphi_{\varepsilon}(\cdot, 0)=\varphi_{0}, \quad \text { in } \Omega .
\end{array}\right.
$$

Then we have

Proposition 3.1 (Global weak solutions to approximating problem). Let $d=2,3$ and $\varepsilon \in(0, \bar{\kappa})$. Suppose that $\varphi_{0} \in V$ with $\Psi\left(\varphi_{0}\right) \in L^{1}(\Omega)$ and $\left|\bar{\varphi}_{0}\right|<1$. Then the following facts hold:

(1) For every $T>0$, there exists at least one global finite energy solution $\left(\boldsymbol{u}_{\varepsilon}, P_{\varepsilon}, \varphi_{\varepsilon}\right)$ to the approximating problem $(\boldsymbol{A P 1})$ on $[0, T]$ such that

$$
\begin{aligned}
& \boldsymbol{u}_{\varepsilon} \in L^{2}\left(0, T ; \mathbf{H}_{\sigma}\right), \quad P_{\varepsilon} \in L^{\frac{8}{5}}\left(0, T ; V_{0}\right), \\
& \varphi_{\varepsilon} \in L^{\infty}(0, T ; V) \cap L^{2}\left(0, T ; H^{3}(\Omega)\right) \cap W^{1, \frac{8}{5}}\left(0, T ; V^{\prime}\right), \\
& \mu_{\varepsilon} \in L^{2}(0, T ; V) .
\end{aligned}
$$

Such a solution satisfies the weak formulation

$$
\left\langle\partial_{t} \varphi_{\varepsilon}, v\right\rangle+\left(\boldsymbol{u}_{\varepsilon} \cdot \nabla \varphi_{\varepsilon}, v\right)+\left(\nabla \mu_{\varepsilon}, \nabla v\right)=0, \quad \forall v \in V, \text { a.e. } t \in(0, T),
$$

where

$$
\boldsymbol{u}_{\varepsilon}=-\nabla P_{\varepsilon}+\mu_{\varepsilon} \nabla \varphi_{\varepsilon}, \quad \mu_{\varepsilon}=-\Delta \varphi_{\varepsilon}+\Psi_{\varepsilon}^{\prime}\left(\varphi_{\varepsilon}\right) \quad \text { a.e. }(x, t) \in \Omega \times(0, T) .
$$

(2) The total mass is conserved

$$
\int_{\Omega} \varphi_{\varepsilon}(t) \mathrm{d} x=\int_{\Omega} \varphi_{0} \mathrm{~d} x, \quad \forall t \in[0, T] .
$$

(3) The pair $\left(\boldsymbol{u}_{\varepsilon}, \varphi_{\varepsilon}\right)$ satisfies the energy inequality

$$
\frac{1}{2}\left\|\nabla \varphi_{\varepsilon}(t)\right\|^{2}+\int_{\Omega} \Psi_{\varepsilon}\left(\varphi_{\varepsilon}(t)\right) \mathrm{d} x \leq \frac{1}{2}\left\|\nabla \varphi_{0}\right\|^{2}+\int_{\Omega} \Psi_{\varepsilon}\left(\varphi_{0}\right) \mathrm{d} x,
$$

for almost every $t \in(0, T)$, and

$$
\int_{0}^{T}\left(\left\|\boldsymbol{u}_{\varepsilon}(\tau)\right\|^{2}+\left\|\nabla \mu_{\varepsilon}(\tau)\right\|^{2}\right) \mathrm{d} \tau \leq \frac{1}{2}\left\|\nabla \varphi_{0}\right\|^{2}+\int_{\Omega} \Psi_{\varepsilon}\left(\varphi_{0}\right) \mathrm{d} x+C,
$$

where $C$ is a constant depending on $\widetilde{\alpha}$ (cf. Lemma 3.1), but is independent of the parameter $\varepsilon$. 
The existence of a global finite energy weak solution to the approximating problem (AP1) on $[0, T]$ can be easily proven by employing a Galerkin approximation scheme (see, e.g., [23, Section 3] and [26]). Indeed, according to the property (AH) in Lemma 3.1, for any $\varepsilon \in(0, \bar{\kappa}]$, the approximating potential $\Psi_{\varepsilon}$ has a quadratic growth as $|s| \rightarrow+\infty$ and $\Psi_{\varepsilon}^{\prime}$ is globally Lipschitz on $\mathbb{R}$.

Remark 3.1. We note that it is sufficient to assume $\varphi_{0} \in V$ to reach the conclusions of Proposition 3.1. Indeed, the additional assumptions such that $\Psi\left(\varphi_{0}\right) \in L^{1}(\Omega)$ and $\left|\overline{\varphi_{0}}\right|<1$ will be necessary to derive uniform estimates with respect to $\varepsilon$ in the subsequent section. Moreover, the estimates for $P_{\varepsilon}$ and $\partial_{t} \varphi_{\varepsilon}$ when $d=2$ can be improved by arguing as in [21, Section 3.4]. Nonetheless, the regularity properties stated above are enough to pass to the limit as $\varepsilon \rightarrow 0^{+}$.

\section{$3.2 \varepsilon$-independent a priori estimates}

In order to pass to the limit as $\varepsilon \rightarrow 0^{+}$, it is necessary to obtain suitable uniform estimates for the approximating solutions $\left(\boldsymbol{u}_{\varepsilon}, P_{\varepsilon}, \varphi_{\varepsilon}\right)$ that are independent of $\varepsilon \in(0, \bar{\kappa}]$.

First, we report the following lemma which turns out to be useful in the sequel (see, e.g., [15] for a proof).

Lemma 3.2. Suppose that $(H)$ is satisfied. For $\varepsilon \in(0, \bar{\kappa}]$, the approximating function $\Psi_{\varepsilon}$ given by (3.1) satisfies the following properties:

$$
\begin{aligned}
& \left|\Psi_{\varepsilon}^{\prime}(s)\right| \leq\left|\Psi^{\prime}(s)\right|, \quad \forall s \in(-1,1), \\
& \Psi_{\varepsilon}(s) \leq \Psi(s), \quad \forall s \in[-1,1] .
\end{aligned}
$$

Now, we are in a position to derive uniform estimates with respect to the approximate parameter $\varepsilon$.

First estimate. According to $(\mathrm{H})$, since $\Psi\left(\varphi_{0}\right) \in L^{1}(\Omega)$, it holds $\left\|\varphi_{0}\right\|_{L^{\infty}(\Omega)} \leq 1$. Then it follows from Lemma 3.2 and the energy inequality (3.7) that, for almost every $t \in(0, T)$,

$$
\frac{1}{2}\left\|\nabla \varphi_{\varepsilon}(t)\right\|^{2}+\int_{\Omega} \Psi_{\varepsilon}\left(\varphi_{\varepsilon}(t)\right) \mathrm{d} x \leq \frac{1}{2}\left\|\nabla \varphi_{0}\right\|^{2}+\int_{\Omega} \Psi\left(\varphi_{0}\right) \mathrm{d} x=\mathcal{E}\left(\varphi_{0}\right) .
$$

Similarly, we infer from (3.8) that

$$
\int_{0}^{T}\left\|\boldsymbol{u}_{\varepsilon}(\tau)\right\|^{2}+\left\|\nabla \mu_{\varepsilon}(\tau)\right\|^{2} \mathrm{~d} \tau \leq \mathcal{E}\left(\varphi_{0}\right)+C
$$

Second estimate. Testing the fourth equation in (3.3) by $-\Delta \varphi_{\varepsilon}$, we obtain

$$
\left\|\Delta \varphi_{\varepsilon}\right\|^{2}-\left(\Psi_{\varepsilon}^{\prime}\left(\varphi_{\varepsilon}\right), \Delta \varphi_{\varepsilon}\right)=-\left(\mu_{\varepsilon}, \Delta \varphi_{\varepsilon}\right) .
$$

Exploiting the integration by parts and the homogeneous Neumann boundary condition for $\varphi_{\varepsilon}$, we get

$$
\left\|\Delta \varphi_{\varepsilon}\right\|^{2}+\left(\Psi_{\varepsilon}^{\prime \prime}\left(\varphi_{\varepsilon}\right) \nabla \varphi_{\varepsilon}, \nabla \varphi_{\varepsilon}\right)=\left(\nabla \mu_{\varepsilon}, \nabla \varphi_{\varepsilon}\right)
$$


Hence, we deduce from $(\mathrm{AH})$ that

$$
\left\|\Delta \varphi_{\varepsilon}\right\|^{2} \leq \alpha\left\|\nabla \varphi_{\varepsilon}\right\|^{2}+\left\|\nabla \mu_{\varepsilon}\right\|\left\|\nabla \varphi_{\varepsilon}\right\|
$$

Taking the square of both sides and integrating in time, we have

$$
\int_{0}^{T}\left\|\Delta \varphi_{\varepsilon}(\tau)\right\|^{4} \mathrm{~d} \tau \leq C(1+T)\left(1+\mathcal{E}\left(\varphi_{0}\right)\right)^{2} .
$$

Third estimate. We provide an uniform estimate for $\partial_{t} \varphi_{\varepsilon}$. By comparison, we easily obtain

$$
\left\|\partial_{t} \varphi_{\varepsilon}\right\|_{V^{\prime}} \leq\left\|\nabla \mu_{\varepsilon}\right\|+\left\|\boldsymbol{u}_{\varepsilon}\right\|\left\|\varphi_{\varepsilon}\right\|_{L^{\infty}(\Omega)} .
$$

Applying the Hölder and Agmon inequalities $(d=3)$, we infer from (3.9)-(3.11) that

$$
\begin{aligned}
\int_{0}^{T} & \left\|\partial_{t} \varphi_{\varepsilon}(\tau)\right\|_{V^{\prime}}^{\frac{8}{5}} \mathrm{~d} \tau \\
\leq & C \int_{0}^{T}\left\|\nabla \mu_{\varepsilon}(\tau)\right\|^{\frac{8}{5}} \mathrm{~d} \tau+C \int_{0}^{T}\left\|\boldsymbol{u}_{\varepsilon}(\tau)\right\|^{\frac{8}{5}}\left\|\varphi_{\varepsilon}(\tau)\right\|_{L^{\infty}(\Omega)}^{\frac{8}{5}} \mathrm{~d} \tau \\
\leq & C \int_{0}^{T}\left\|\nabla \mu_{\varepsilon}(\tau)\right\|^{\frac{8}{5}} \mathrm{~d} \tau+C \int_{0}^{T}\left\|\boldsymbol{u}_{\varepsilon}(\tau)\right\|^{\frac{8}{5}}\left\|\varphi_{\varepsilon}(\tau)\right\|_{V}^{\frac{4}{5}}\left\|\varphi_{\varepsilon}(\tau)\right\|_{H^{2}(\Omega)}^{\frac{4}{5}} \mathrm{~d} \tau \\
\leq & C \int_{0}^{T}\left(1+\left\|\nabla \mu_{\varepsilon}(\tau)\right\|^{2}\right) \mathrm{d} \tau \\
& +C\left\|\varphi_{\varepsilon}\right\|_{L^{\infty}(0, T ; V)}^{\frac{4}{5}}\left(\int_{0}^{T}\left\|\boldsymbol{u}_{\varepsilon}(\tau)\right\|^{2} \mathrm{~d} \tau\right)^{\frac{4}{5}}\left(\int_{0}^{T}\left\|\varphi_{\varepsilon}(\tau)\right\|_{H^{2}(\Omega)}^{4} \mathrm{~d} \tau\right)^{\frac{1}{5}} \\
\leq & C(1+T)\left(1+\mathcal{E}\left(\varphi_{0}\right)\right)^{\frac{8}{5}} .
\end{aligned}
$$

Fourth estimate. We derive an uniform estimate for $\left\|\mu_{\varepsilon}\right\|_{V}$. On account of the Poincaré-Wirtinger inequality

$$
\left\|\mu_{\varepsilon}\right\| \leq C_{P}\left(\left\|\nabla \mu_{\varepsilon}\right\|+\left|\overline{\mu_{\varepsilon}}\right|\right)
$$

and in light of (3.10), it is sufficient to estimate the mean value $\overline{\mu_{\varepsilon}}$. On the other hand, since

$$
\int_{\Omega} \mu_{\varepsilon} \mathrm{d} x=\int_{\Omega} \Psi_{\varepsilon}^{\prime}\left(\varphi_{\varepsilon}\right) \mathrm{d} x
$$

it remains to find an uniform control of $\Psi_{\varepsilon}^{\prime}\left(\varphi_{\varepsilon}\right)$ in $L^{1}(\Omega)$. To this aim, we recall the well-known inequality for approximating functions of singular potentials satisfying the assumption (H) (see, e.g., [15], [24] and [28] for the proof)

$$
\left\|S_{\varepsilon}^{\prime}\left(\varphi_{\varepsilon}\right)\right\|_{L^{1}(\Omega)} \leq C \int_{\Omega}\left(\varphi_{\varepsilon}-\bar{\varphi}_{0}\right)\left(S_{\varepsilon}^{\prime}\left(\varphi_{\varepsilon}\right)-\overline{S_{\varepsilon}^{\prime}\left(\varphi_{\varepsilon}\right)}\right) \mathrm{d} x+C
$$

where $C$ may depend on $\bar{\varphi}_{0}$ and $\Psi$ but is independent of $\varepsilon$. Then, testing the fourth equation in (3.3) by $\varphi_{\varepsilon}-\bar{\varphi}_{0}$, and using the integration by parts together with the boundary condition on $\varphi_{\varepsilon}$ and Poincaré's inequality (2.1), we find 


$$
\begin{aligned}
& \left\|\nabla \varphi_{\varepsilon}\right\|^{2}+\int_{\Omega}\left(\varphi_{\varepsilon}-\bar{\varphi}_{0}\right) S_{\varepsilon}^{\prime}\left(\varphi_{\varepsilon}\right) \mathrm{d} x \\
& \quad=\int_{\Omega} \mu_{\varepsilon}\left(\varphi_{\varepsilon}-\bar{\varphi}_{0}\right) \mathrm{d} x+\Theta_{0} \int_{\Omega} \varphi_{\varepsilon}\left(\varphi_{\varepsilon}-\bar{\varphi}_{0}\right) \mathrm{d} x \\
& \quad=\int_{\Omega}\left(\mu_{\varepsilon}-\bar{\mu}_{\varepsilon}\right)\left(\varphi_{\varepsilon}-\bar{\varphi}_{0}\right) \mathrm{d} x+C\left\|\nabla \varphi_{\varepsilon}\right\|^{2} \\
& \quad \leq C\left(\left\|\nabla \mu_{\varepsilon}\right\|\left\|\nabla \varphi_{\varepsilon}\right\|+\left\|\nabla \varphi_{\varepsilon}\right\|^{2}\right) .
\end{aligned}
$$

Hence, collecting (3.14) and (3.15), and using (3.9) and (3.10), after an integration in time we get

$$
\begin{aligned}
\int_{0}^{T}\left\|S_{\varepsilon}^{\prime}\left(\varphi_{\varepsilon}(\tau)\right)\right\|_{L^{1}(\Omega)}^{2} \mathrm{~d} \tau & \leq C \int_{0}^{T}\left(\left\|\nabla \mu_{\varepsilon}(\tau)\right\|^{2}\left\|\nabla \varphi_{\varepsilon}(\tau)\right\|^{2}+\left\|\nabla \varphi_{\varepsilon}(\tau)\right\|^{4}\right) \mathrm{d} \tau \\
& \leq C(1+T)\left(1+\mathcal{E}\left(\varphi_{0}\right)\right)^{2}
\end{aligned}
$$

which together with (3.9) yields

$$
\begin{aligned}
\int_{0}^{T}\left|\overline{\mu_{\varepsilon}}(\tau)\right|^{2} \mathrm{~d} \tau & \leq 2|\Omega|^{-2} \int_{0}^{T}\left(\left\|S_{\varepsilon}^{\prime}\left(\varphi_{\varepsilon}(\tau)\right)\right\|_{L^{1}(\Omega)}^{2}+\Theta_{0}^{2}\left\|\varphi_{\varepsilon}(\tau)\right\|_{L^{1}(\Omega)}^{2}\right) \mathrm{d} \tau \\
& \leq C(1+T)\left(1+\mathcal{E}\left(\varphi_{0}\right)\right)^{2}
\end{aligned}
$$

The above estimate together with (3.10) and (3.13) implies

$$
\int_{0}^{T}\left\|\mu_{\varepsilon}(\tau)\right\|_{V}^{2} \mathrm{~d} \tau \leq C(1+T)\left(1+\mathcal{E}\left(\varphi_{0}\right)\right)^{2} .
$$

Fifth estimate. We aim to derive a uniform estimate for the pressure $P_{\varepsilon}$. It follows from the Darcy's equation for $\boldsymbol{u}_{\varepsilon}$, the Gagliardo-Nirenberg inequality $(d=3)$ and the estimates (3.9), (3.10), (3.11) and (3.17) that

$$
\begin{aligned}
\int_{0}^{T} & \left\|\nabla P_{\varepsilon}(\tau)\right\|^{\frac{8}{5}} \mathrm{~d} \tau \\
\leq & C \int_{0}^{T}\left(\left\|\boldsymbol{u}_{\varepsilon}(\tau)\right\|^{\frac{8}{5}}+\left\|\nabla \varphi_{\varepsilon}(\tau)\right\|_{\mathbf{L}^{3}(\Omega)}^{\frac{8}{5}}\left\|\mu_{\varepsilon}(\tau)\right\|_{L^{6}(\Omega)}^{\frac{8}{5}}\right) \mathrm{d} \tau \\
\leq & C \int_{0}^{T}\left(1+\left\|\boldsymbol{u}_{\varepsilon}(\tau)\right\|^{2}\right) \mathrm{d} \tau+C\left\|\varphi_{\varepsilon}(t)\right\|_{L^{\infty}(0, T ; V)}^{\frac{4}{5}} \int_{0}^{T}\left\|\varphi_{\varepsilon}(\tau)\right\|_{H^{2}(\Omega)}^{\frac{4}{5}}\left\|\mu_{\varepsilon}\right\|_{V}^{\frac{8}{5}} \mathrm{~d} \tau \\
\leq & C \int_{0}^{T}\left(1+\left\|\boldsymbol{u}_{\varepsilon}(\tau)\right\|^{2}\right) \mathrm{d} \tau \\
& +C\left\|\varphi_{\varepsilon}\right\|_{L^{\infty}(0, T ; V)}^{\frac{4}{5}}\left(\int_{0}^{T}\left\|\varphi_{\varepsilon}(\tau)\right\|_{H^{2}(\Omega)}^{4} \mathrm{~d} \tau\right)^{\frac{1}{5}}\left(\int_{0}^{T}\left\|\mu_{\varepsilon}(\tau)\right\|_{V}^{2} \mathrm{~d} \tau\right)^{\frac{4}{5}} \\
\leq & C(1+T)\left(1+\mathcal{E}\left(\varphi_{0}\right)\right)^{\frac{12}{5}}
\end{aligned}
$$

Collecting all the above estimates, we conclude that

$$
\left\|\varphi_{\varepsilon}\right\|_{L^{\infty}(0, T ; V)} \leq C
$$




$$
\begin{aligned}
& \left\|\varphi_{\varepsilon}\right\|_{L^{4}\left(0, T ; H^{2}(\Omega)\right)} \leq C, \\
& \left\|\partial_{t} \varphi_{\varepsilon}\right\|_{L^{\frac{8}{5}}\left(0, T ; V^{\prime}\right)} \leq C, \\
& \left\|\boldsymbol{u}_{\varepsilon}\right\|_{L^{2}\left(0, T ; \mathbf{H}_{\sigma}\right)} \leq C, \\
& \left\|\mu_{\varepsilon}\right\|_{L^{2}(0, T ; V)} \leq C, \\
& \left\|P_{\varepsilon}\right\|_{L^{\frac{8}{5}\left(0, T ; V_{0}\right)}} \leq C,
\end{aligned}
$$

where the constant $C>0$ depends on the initial energy $\mathcal{E}\left(\varphi_{0}\right)$, the form of $\Psi, \Omega$ and coefficients of the system, but is independent of $\varepsilon$.

\subsection{Proof of Theorem 2.1}

We are now in a position to prove Theorem 2.1. The proof consists of several steps.

Step 1. Preliminary convergence results. Thanks to the uniform estimates (3.19)-(3.24), letting $\varepsilon \rightarrow 0^{+}$, the following weak convergence results hold (up to a subsequence):

$$
\begin{array}{ll}
\varphi_{\varepsilon} \rightarrow \varphi, & \text { weakly star in } L^{\infty}(0, T ; V), \\
\varphi_{\varepsilon} \rightarrow \varphi, & \text { weakly in } L^{4}\left(0, T ; H^{2}(\Omega)\right), \\
\partial_{t} \varphi_{\varepsilon} \rightarrow \partial_{t} \varphi, & \text { weakly in } L^{\frac{8}{5}}\left(0, T ; V^{\prime}\right), \\
\mu_{\varepsilon} \rightarrow \mu, & \text { weakly in } L^{2}(0, T ; V), \\
\boldsymbol{u}_{\varepsilon} \rightarrow \boldsymbol{u}, & \text { weakly in } L^{2}\left(0, T ; \mathbf{H}_{\sigma}\right), \\
P_{\varepsilon} \rightarrow P, & \text { weakly in } L^{\frac{8}{5}}\left(0, T ; V_{0}\right),
\end{array}
$$

Besides, on account of the Aubin-Lions compactness lemma, we have

$$
\varphi_{\varepsilon} \rightarrow \varphi, \quad \text { strongly in } \mathcal{C}([0, T], H) \cap L^{4}\left(0, T ; W^{1, r}(\Omega)\right),
$$

for $r \in[1,6)$ when $d=3$ and $r \in[1,+\infty)$ when $d=2$, which also implies the pointwise convergence

$$
\varphi_{\varepsilon} \rightarrow \varphi, \quad \text { a.e. in } \Omega \times(0, T) .
$$

Step 2. $L^{\infty}$-estimate for $\varphi$. On account of the singular potential $\Psi$, we shall prove that the limit function $\varphi$ fulfills

$$
\varphi \in L^{\infty}(\Omega \times(0, T)) \text { and }|\varphi(x, t)|<1 \quad \text { a.e. in } \Omega \times(0, T) .
$$

It follows from (3.16) that

$$
\left\|S_{\varepsilon}^{\prime}\left(\varphi_{\varepsilon}\right)\right\|_{L^{1}(\Omega \times(0, T))} \leq C,
$$

with $C$ independent of $\varepsilon$. By the definition of $S_{\varepsilon}$ and the assumption (H), there exists a constant $\varrho \in(0, \bar{\kappa})$ such that for all $\varepsilon \in(0, \varrho], S_{\varepsilon}^{\prime}(s) \geq 1$ for $s \in[1-\varrho,+\infty)$ and $S_{\varepsilon}^{\prime}(s) \leq-1$ for $s \in(-\infty,-1+\varrho]$ and $S_{\varepsilon}^{\prime}(s)$ is monotone increasing for $s \in \mathbb{R}$. Then we introduce the sets

$$
E_{\varrho}^{\varepsilon}=\left\{(x, t) \in \Omega \times(0, T):\left|\varphi_{\varepsilon}(x, t)\right|>1-\varrho\right\}, \quad \varepsilon \in(0, \varrho],
$$




$$
E_{\varrho}=\{(x, t) \in \Omega \times(0, T):|\varphi(x, t)|>1-\varrho\} .
$$

From the pointwise convergence of $\varphi_{\varepsilon}$ and Fatou's Lemma, we infer that for any fixed $\varrho$,

$$
\operatorname{meas}\left(E_{\varrho}\right) \leq \liminf _{\varepsilon \rightarrow 0^{+}} \operatorname{meas}\left(E_{\varrho}^{\varepsilon}\right) .
$$

At the same time, when $\varepsilon \in(0, \varrho]$, we deduce from (3.34) that

$$
\min \left\{S^{\prime}(1-\varrho),-S^{\prime}(-1+\varrho)\right\} \operatorname{meas}\left(E_{\varrho}^{\varepsilon}\right) \leq\left\|S_{\varepsilon}^{\prime}\left(\varphi_{\varepsilon}\right)\right\|_{L^{1}(\Omega \times(0, T))} \leq C,
$$

where the constant $C$ does not depend on $\varrho$ and $\varepsilon$. Therefore, we have

$$
\operatorname{meas}\left(E_{\varrho}\right) \leq \frac{C}{\min \left\{S^{\prime}(1-\varrho),-S^{\prime}(-1+\varrho)\right\}} .
$$

Passing to the limit as $\varrho \rightarrow 0^{+}$, we deduce that

$$
\operatorname{meas}(\{(x, t) \in \Omega \times(0, T):|\varphi(x, t)| \geq 1\})=0,
$$

which yields the conclusion (3.33).

We can further conclude that $\varphi \in L^{\infty}\left(0, T ; L^{\infty}(\Omega)\right)$. Indeed, since $\varphi \in L^{4}\left(0, T ; H^{2}(\Omega)\right)$, by the Sobolev embedding theorem $H^{2}(\Omega) \hookrightarrow L^{\infty}(\Omega)$, it follows that the function $\varphi$ is measurable on $[0, T]$ with values in $L^{\infty}(\Omega)$.

Step 3. Passage to the limit as $\varepsilon \rightarrow 0^{+}$. The $L^{\infty}$-estimate (3.33) together with the pointwise convergence of $\varphi_{\varepsilon}$ and the uniform convergence of $\Psi_{\varepsilon}^{\prime}$ to $\Psi^{\prime}$ on every compact set in $(-1,1)$ entails that

$$
\Psi_{\varepsilon}^{\prime}\left(\varphi_{\varepsilon}\right) \rightarrow \Psi^{\prime}(\varphi) \text { a.e. }(x, t) \in \Omega \times(0, T),
$$

as $\varepsilon \rightarrow 0^{+}$. Besides, by comparison in the equation for $\mu_{\varepsilon}$ (see (3.6)) and owing to the estimates (3.20) and (3.23), we have

$$
\left\|\Psi_{\varepsilon}^{\prime}\left(\varphi_{\varepsilon}\right)\right\|_{L^{2}(0, T ; H)} \leq C
$$

uniformly in $\varepsilon$. Hence, up to a subsequence, it holds

$$
\Psi_{\varepsilon}^{\prime}\left(\varphi_{\varepsilon}\right) \rightarrow \Psi^{\prime}(\varphi), \quad \text { weakly in } L^{2}(0, T ; H) .
$$

On the other hand, it follows from (3.28), (3.29) and (3.31) with $r=4$ that

$$
\mu_{\varepsilon} \nabla \varphi_{\varepsilon} \rightarrow \mu \nabla \varphi, \quad \text { weakly in } L^{\frac{4}{3}}(\Omega \times(0, T)) .
$$

In a similar manner, we have

$$
\boldsymbol{u}_{\varepsilon} \cdot \nabla \varphi_{\varepsilon} \rightarrow \boldsymbol{u} \cdot \nabla \varphi, \quad \text { weakly in } L^{\frac{4}{3}}(\Omega \times(0, T)) .
$$

On account of (3.25)-(3.30) and (3.35)-(3.37), we are able to pass to the limit as $\varepsilon \rightarrow 0^{+}$ (up to a subsequence) in the weak formulation (3.5)-(3.6) for $\left(\boldsymbol{u}_{\varepsilon}, P_{\varepsilon}, \varphi_{\varepsilon}\right)$ and the limit triple $(\boldsymbol{u}, P, \varphi)$ fulfills $(2.17)-(2.18)$. 
Step 4. Further regularity properties. We establish some further regularity results for the global weak solution with finite energy $(\boldsymbol{u}, P, \varphi)$ by making use of the estimates (3.20) and (3.33). First, it follows from (3.29) and (3.33) that

$$
\boldsymbol{u} \cdot \nabla \varphi \in L^{2}\left(0, T ; V^{\prime}\right)
$$

Then by comparison in (2.17), we immediately see that

$$
\varphi_{t} \in L^{2}\left(0, T ; V^{\prime}\right)
$$

Next, an application of Lemma 7.4 with $f=\mu+\Theta_{0} \varphi$ gives

$$
\begin{gathered}
\int_{0}^{T}\|\varphi(\tau)\|_{W^{2, p}(\Omega)}^{2} \mathrm{~d} \tau+\int_{0}^{T}\left\|S^{\prime}(\varphi(\tau))\right\|_{L^{p}(\Omega)}^{2} \mathrm{~d} \tau \\
\leq C \int_{0}^{T}\left(1+\|\mu(\tau)\|_{V}^{2}+\|\varphi(\tau)\|_{V}^{2}\right) \mathrm{d} \tau \leq C,
\end{gathered}
$$

where $p=6$ if $d=3$ and $2 \leq p<\infty$ if $d=2$. We recall that by the Gagliardo-Nirenberg inequality

$$
\|\nabla \varphi\|_{\mathbf{L}^{\infty}(\Omega)} \leq \begin{cases}C\|\varphi\|_{L^{\infty}(\Omega)}^{\frac{r-2}{2(r-1)}}\|\varphi\|_{W^{2, r}(\Omega)}^{\frac{r}{2(r-1)}}, & \text { for } d=2, r>2 \\ C\|\varphi\|_{L^{\infty}(\Omega)}^{\frac{1}{3}}\|\varphi\|_{W^{2,6}(\Omega)}^{\frac{2}{3}}, & \text { for } d=3\end{cases}
$$

and by interpolation between $L^{p}$-spaces

$$
\|\Delta \varphi\|_{L^{3}(\Omega)} \leq C\|\varphi\|_{H^{2}(\Omega)}^{\frac{2(r-3)}{3(r-2)}}\|\varphi\|_{W^{2, r}(\Omega)}^{\frac{r}{3(r-2)}}, \quad \text { for } r \geq 3 .
$$

Applying the curl operator to the Darcy's equation for $\boldsymbol{u}$ and exploiting the particular form of the Korteweg force, we deduce that

$$
\|\nabla \times \boldsymbol{u}\|=\|\nabla \mu \times \nabla \varphi\| \leq C\|\nabla \mu\|\|\nabla \varphi\|_{\mathbf{L}^{\infty}(\Omega)},
$$

Then by (3.33) and (3.41), we find

$$
\begin{array}{cl}
\|\nabla \times \boldsymbol{u}\|^{\frac{4(r-1)}{3 r-2}} \leq C\|\nabla \mu\|^{\frac{4(r-1)}{3 r-2}}\|\varphi\|_{W^{2, r}(\Omega)}^{\frac{2 r}{3 r-2}} \leq C\|\nabla \mu\|^{2}+C\|\varphi\|_{W^{2, r}(\Omega)}^{2}, & \text { for } d=2, r>2, \\
\|\nabla \times \boldsymbol{u}\|^{\frac{6}{5}} \leq C\|\nabla \mu\|^{\frac{6}{5}}\|\varphi\|_{W^{2,6}(\Omega)}^{\frac{4}{5}} \leq C\|\nabla \mu\|^{2}+C\|\varphi\|_{W^{2,6}(\Omega)}^{2}, \quad & \text { for } d=3 .
\end{array}
$$

Besides, according to the Neumann problem (2.7) with $\boldsymbol{u}=\mu \nabla \varphi$, we have

$$
\|\Delta P\|=\|\nabla \cdot(\mu \nabla \varphi)\| \leq C\|\nabla \mu\|\|\nabla \varphi\|_{\mathbf{L}^{\infty}(\Omega)}+C\|\mu\|_{L^{6}(\Omega)}\|\Delta \varphi\|_{L^{3}(\Omega)} .
$$

Hence, we get

$$
\begin{aligned}
\|\Delta P\|^{\frac{6(r-2)}{5 r-9}} & \leq C\|\nabla \mu\|^{\frac{6(r-2)}{5 r-9}}\|\varphi\|_{W^{2, r}(\Omega)}^{\frac{3 r(r-2)}{5 r-1)}}+C\|\mu\|_{V}^{\frac{6(r-2)}{5 r-9}}\|\varphi\|_{H^{2}(\Omega)}^{\frac{4(r-3)}{5 r-9}}\|\varphi\|_{W^{2, r}(\Omega)}^{\frac{2 r}{5 r-9}} \\
& \leq C\|\mu\|_{V}^{2}+C\|\varphi\|_{W^{2, r}(\Omega)}^{2}+C\|\varphi\|_{H^{2}(\Omega)}^{4}+C, \quad \text { for } d=2, r \geq 3, \\
\|\Delta P\|^{\frac{8}{7}} & \leq C\|\nabla \mu\|^{\frac{8}{7}}\|\varphi\|_{W^{2,6}(\Omega)}^{\frac{16}{21}}+C\|\mu\|_{V}^{\frac{8}{7}}\|\varphi\|_{W^{2,6}(\Omega)}^{\frac{4}{7}}\|\varphi\|_{H^{2}(\Omega)}^{\frac{4}{7}}
\end{aligned}
$$




$$
\leq C\|\mu\|_{V}^{2}+C\|\varphi\|_{W^{2,6}(\Omega)}^{2}+C\|\varphi\|_{H^{2}(\Omega)}^{4}+C, \quad \text { for } d=3
$$

The above estimates together with (3.19), (3.23), (3.40) and the inequality (2.8) yield that

$$
\|\boldsymbol{u}\|_{L^{s}(0, T ; \mathbf{V})} \leq C, \quad\|P\|_{L^{q}\left(0, T ; H^{2}(\Omega)\right)} \leq C,
$$

where $s=\frac{6}{5}$ if $d=3$ and $1 \leq s<\frac{4}{3}$ if $d=2, q=\frac{8}{7}$ is $d=3$ and $1 \leq q<\frac{6}{5}$ if $d=2$.

Step 5. Mass conservation and energy identity. For every global weak solution, taking $v=1$ in (2.17), we have

$$
\frac{\mathrm{d}}{\mathrm{d} t} \int_{\Omega} \varphi \mathrm{d} x=\left\langle\varphi_{t}, 1\right\rangle=0
$$

which implies the mass conservation (2.21).

Next, due to the regularity properties of the finite energy weak solution (see (3.29), (3.38), (3.39) and (3.45)), for a.e. $t \in(0, T)$ we are able to test the Darcy's equation by $\boldsymbol{u}$ and to take $v=\mu$ in (2.18) to get

$$
\begin{aligned}
& \|\boldsymbol{u}\|^{2}=\int_{\Omega}(\mu \nabla \varphi) \cdot \boldsymbol{u} \mathrm{d} x \\
& \left\langle\varphi_{t}, \mu\right\rangle+\int_{\Omega}(\boldsymbol{u} \cdot \nabla \varphi) \mu \mathrm{d} x+\|\nabla \mu\|^{2}=0 .
\end{aligned}
$$

Adding (3.46) and (3.47) together, we get

$$
\left\langle\varphi_{t}, \mu\right\rangle+\|\boldsymbol{u}\|^{2}+\|\nabla \mu\|^{2}=0, \quad \text { for a.e. } t \in(0, T) .
$$

On the other hand, we consider the functional

$$
\mathcal{S}(u)=\frac{1}{2}\|\nabla u\|^{2}+\int_{\Omega} S(u) \mathrm{d} x
$$

defined on $V$. It is well-known that $\mathcal{S}$ is proper, lower-semicontinuous, convex with domain

$$
\operatorname{dom}(\mathcal{S})=\{u \in V: u(x) \in[-1,1] \text { a.e. } x \in \Omega\} .
$$

Being the subgradient of $\mathcal{S}$ equal to $\partial \mathcal{S}(u)=-\Delta u+S^{\prime}(u)$, and on account of the regularity $-\Delta \varphi+S^{\prime}(\varphi) \in L^{2}(0, T ; V)$ and $\varphi_{t} \in L^{2}\left(0, T ; V^{\prime}\right)$ of a finite energy weak solution, we learn from [30, Lemma 4.1] that

$$
\begin{aligned}
\frac{\mathrm{d}}{\mathrm{d} t} \mathcal{E}(\varphi(t))= & \frac{\mathrm{d}}{\mathrm{d} t} \mathcal{S}(\varphi(t))-\frac{\mathrm{d}}{\mathrm{d} t} \frac{\Theta_{0}}{2}\|\varphi(t)\|^{2} \\
& =\left\langle\varphi_{t}(t),-\Delta \varphi(t)+S^{\prime}(\varphi(t))\right\rangle-\Theta_{0}\left\langle\varphi_{t}(t), \varphi(t)\right\rangle \\
& =\left\langle\varphi_{t}(t), \mu(t)\right\rangle, \quad \text { for a.e. } t \in(0, T) .
\end{aligned}
$$

Here, we have also used the standard chain rule in $H^{1}\left(0, T ; V^{\prime}\right) \cap L^{2}(0, T ; V)$. As a consequence, the required energy identity $(2.20)$ holds, which yields that $\mathcal{E}(\varphi(t))$ is absolutely continuous on $[0, T]$ and fulfills

$$
\mathcal{E}(\varphi(t))+\int_{0}^{t}\|\boldsymbol{u}(\tau)\|^{2} \mathrm{~d} \tau+\int_{0}^{t}\|\nabla \mu(\tau)\|^{2} \mathrm{~d} \tau=\mathcal{E}\left(\varphi_{0}\right), \quad \forall t \geq 0
$$


Lastly, we see from (3.25) and (3.27) that $\varphi \in \mathcal{C}_{w}([0, T], V)$ as well as $\varphi \in \mathcal{C}([0, T], H)$. At the same time, thanks to the continuity of $\mathcal{S}(\varphi(t))$ and the convexity of $S$, we obtain that $t \rightarrow\|\nabla \varphi(t)\|^{2}$ is continuous (cf. [1, Theorem 6]). As a result, this gives $\varphi \in \mathcal{C}([0, T], V)$. The proof of Theorem 2.1 is complete.

Remark 3.2. It is worth pointing out that the hypothesis on the growth of $S^{\prime \prime}$ assumed in $(H)$ can be removed by working with an approximation family of singular potentials proposed in [17].

\subsection{Proof of Theorem 2.2}

We conclude this section by deducing the dissipative estimates stated in Theorem 2.2.

Testing the equation for $\mu$ in (2.18) by $\varphi-\bar{\varphi}_{0}$ and using the mass conservation (2.21), we get

$$
\|\nabla \varphi\|^{2}+\int_{\Omega} \Psi^{\prime}(\varphi)\left(\varphi-\bar{\varphi}_{0}\right) \mathrm{d} x=\int_{\Omega}(\mu-\bar{\mu})\left(\varphi-\bar{\varphi}_{0}\right) \mathrm{d} x
$$

Recalling the basic inequality for a singular potential satisfying $(\mathrm{H})$

$$
\Psi(s) \leq \Psi(w)+\Psi^{\prime}(s)(s-w)+\frac{\alpha}{2}(s-w)^{2}, \quad \forall s, w \in(-1,1),
$$

and exploiting the regularity $|\varphi(x, t)|<1$ for almost every $(x, t) \in \Omega \times(0,+\infty)$, we find

$$
\begin{aligned}
\int_{\Omega} \Psi^{\prime}(\varphi)\left(\varphi-\bar{\varphi}_{0}\right) \mathrm{d} x & \geq \int_{\Omega} \Psi(\varphi) \mathrm{d} x-\Psi\left(\bar{\varphi}_{0}\right)|\Omega|-\frac{\alpha}{2}\left\|\varphi-\bar{\varphi}_{0}\right\|^{2} \\
& \geq \int_{\Omega} \Psi(\varphi) \mathrm{d} x-C,
\end{aligned}
$$

where $C>0$ depends on $\Omega$ but is independent of $\varphi_{0}$. Inserting the above inequality into (3.50) and applying the Poincaré inequality (2.1), we infer that

$$
\frac{1}{2}\|\nabla \varphi\|^{2}+\int_{\Omega} \Psi(\varphi) \mathrm{d} x \leq C\|\nabla \mu\|^{2}+C .
$$

Hence, in light of the energy identity (2.20), we obtain

$$
\frac{\mathrm{d}}{\mathrm{d} t} \mathcal{E}(\varphi)+\omega \mathcal{E}(\varphi)+\|\boldsymbol{u}\|^{2}+\frac{1}{2}\|\nabla \mu\|^{2} \leq C,
$$

where $\omega, C$ are positive constants independent of $\varphi_{0}$. An application of the Gronwall lemma yields, for all $t \geq 0$,

$$
\mathcal{E}(\boldsymbol{u}(t), \varphi(t))+\int_{t}^{t+1}\left(\|\boldsymbol{u}(\tau)\|^{2}+\|\nabla \mu(\tau)\|^{2}\right) \mathrm{d} \tau \leq C \mathcal{E}\left(\boldsymbol{u}_{0}, \varphi_{0}\right) \mathrm{e}^{-\omega t}+C,
$$

In particular, this gives

$$
\|\varphi(t)\|_{V}^{2} \leq C \mathcal{E}\left(\boldsymbol{u}_{0}, \varphi_{0}\right) \mathrm{e}^{-\omega t}+C, \quad \forall t \geq 0 .
$$

Next, by Lemma 7.3 with $f=\mu+\Theta_{0} \varphi$, we learn from (3.52), (3.53) that

$$
\int_{t}^{t+1}\|\Delta \varphi(\tau)\|^{4} \mathrm{~d} \tau \leq\left(C \mathcal{E}\left(\boldsymbol{u}_{0}, \varphi_{0}\right) \mathrm{e}^{-\omega t}+C\right)^{2}, \quad \forall t \geq 0 .
$$


Then, by repeating the same argument exploited in the proof of Theorem 2.1 to get an uniform control of $\mu$ in $V$ (cf. (3.17)), we obtain

$$
\int_{t}^{t+1}\|\mu(\tau)\|_{V}^{2} \mathrm{~d} \tau \leq\left(C \mathcal{E}\left(\boldsymbol{u}_{0}, \varphi_{0}\right) \mathrm{e}^{-\omega t}+C\right)^{2}, \quad \forall t \geq 0 .
$$

Here, the constant $C$ depends on the total mass of the initial datum. Therefore, by Lemma 7.4 with the same choice of $f=\mu+\Theta_{0} \varphi$, we find

$$
\int_{t}^{t+1}\left(\|\varphi(\tau)\|_{W^{2, p}(\Omega)}^{2}+\left\|S^{\prime}(\varphi)\right\|_{L^{p}(\Omega)}^{2}\right) \mathrm{d} \tau \leq\left(C \mathcal{E}\left(\boldsymbol{u}_{0}, \varphi_{0}\right) \mathrm{e}^{-\omega t}+C\right)^{2}, \quad \forall t \geq 0 .
$$

where $p=6$ if $d=3$ and for any $p \geq 2$ if $d=2$.

The proof of Theorem 2.2 is complete.

\section{Uniqueness of Global Weak Solutions in Two Dimensions}

In this section, we prove the uniqueness of global weak solutions with finite energy to problem (1.1)-(1.2) when the spatial dimension is two.

Proof of Theorem 2.3. Let $\left(\boldsymbol{u}_{1}, P_{1}, \varphi_{1}\right)$ and $\left(\boldsymbol{u}_{2}, P_{2}, \varphi_{2}\right)$ be two global finite energy solutions to problem (1.1)-(1.2) on $[0, T]$ with initial data $\varphi_{01}$ and $\varphi_{02}$, respectively. Their difference denoted by $(\varphi, P, \boldsymbol{u})=\left(\boldsymbol{u}_{1}-\boldsymbol{u}_{2}, P_{1}-P_{2}, \varphi_{1}-\varphi_{2}\right)$ solves

$$
\left\langle\varphi_{t}, v\right\rangle-\left(\boldsymbol{u}_{1} \varphi, \nabla v\right)-\left(\boldsymbol{u} \varphi_{2}, \nabla v\right)+(\nabla \mu, \nabla v)=0, \quad \forall v \in V
$$

for almost every $t \in(0, T)$, where (cf. Remark 2.2)

$$
\left\{\begin{array}{l}
\boldsymbol{u}=-\Pi\left(\operatorname{div}\left(\nabla \varphi_{1} \otimes \nabla \varphi\right)+\operatorname{div}\left(\nabla \varphi \otimes \nabla \varphi_{2}\right)\right), \\
\mu=-\Delta \varphi+\left(\Psi^{\prime}\left(\varphi_{1}\right)-\Psi^{\prime}\left(\varphi_{2}\right)\right) .
\end{array}\right.
$$

Thanks to the mass conservation and $\bar{\varphi}_{01}=\bar{\varphi}_{02}$, we observe that $\bar{\varphi}=0$ for all $t \geq 0$. By Theorem 2.1, we also know that

$$
\left\|\varphi_{i}(t)\right\|_{V}+\left\|\varphi_{i}(t)\right\|_{L^{\infty}(\Omega)} \leq C, \quad \forall t \in[0, T], \quad i=1,2 .
$$

Taking $v=\mathcal{N} \varphi$ in (4.1), and using (2.2), we get

$$
\frac{1}{2} \frac{\mathrm{d}}{\mathrm{d} t}\|\varphi\|_{V_{0}^{\prime}}^{2}+(\mu, \varphi)=\left(\boldsymbol{u}_{1} \varphi, \nabla \mathcal{N} \varphi\right)+\left(\boldsymbol{u} \varphi_{2}, \nabla \mathcal{N} \varphi\right) .
$$

Using integration by parts and the homogeneous Neumann boundary condition for $\varphi$, and making use of $(\mathrm{H})$ and (2.4), we have

$$
\begin{aligned}
(\mu, \varphi) & =\|\nabla \varphi\|^{2}+\left(\Psi^{\prime}\left(\varphi_{1}\right)-\Psi^{\prime}\left(\varphi_{2}\right), \varphi\right) \\
& \geq\|\nabla \varphi\|^{2}-\alpha\|\varphi\|^{2} \\
& \geq \frac{1}{2}\|\nabla \varphi\|^{2}-C\|\varphi\|_{V_{0}^{\prime}}^{2} .
\end{aligned}
$$


Then, the differential equality (4.4) turns into

$$
\frac{1}{2} \frac{\mathrm{d}}{\mathrm{d} t}\|\varphi\|_{V_{0}^{\prime}}^{2}+\frac{1}{2}\|\nabla \varphi\|^{2} \leq C\|\varphi\|_{V_{0}^{\prime}}^{2}+I+J
$$

where

$$
I=\left(\boldsymbol{u}_{1} \varphi, \nabla \mathcal{N} \varphi\right) \quad \text { and } \quad J=\left(\boldsymbol{u} \varphi_{2}, \nabla \mathcal{N} \varphi\right) .
$$

Firstly, by (2.1), (2.4), (2.5) and (2.9), we control $I$ as follows

$$
\begin{aligned}
I & \leq\left\|\boldsymbol{u}_{1}\right\|\|\varphi\|_{L^{4}(\Omega)}\|\nabla \mathcal{N} \varphi\|_{\mathbf{L}^{4}(\Omega)} \\
& \leq C\left\|\boldsymbol{u}_{1}\right\|\|\varphi\|^{\frac{1}{2}}\|\varphi\|_{V}^{\frac{1}{2}}\|\nabla \mathcal{N} \varphi\|^{\frac{1}{2}}\|\nabla \mathcal{N} \varphi\|_{\mathbf{V}}^{\frac{1}{2}} \\
& \leq C\left\|\boldsymbol{u}_{1}\right\|\|\varphi\|_{V_{0}^{\prime}}^{\frac{1}{2}}\|\varphi\|\|\nabla \varphi\|^{\frac{1}{2}} \\
& \leq C\left\|\boldsymbol{u}_{1}\right\|\|\varphi\|_{V_{0}^{\prime}}\|\nabla \varphi\| \\
& \leq \frac{1}{8}\|\nabla \varphi\|^{2}+C\left\|\boldsymbol{u}_{1}\right\|^{2}\|\varphi\|_{V_{0}^{\prime}}^{2} .
\end{aligned}
$$

Next, we take care of $J$. To this aim, by means of $(4.2), J$ can be rewritten as

$$
\begin{aligned}
J & =-\left(\Pi\left(\operatorname{div}\left(\nabla \varphi_{1} \otimes \nabla \varphi\right)+\operatorname{div}\left(\nabla \varphi \otimes \nabla \varphi_{2}\right)\right), \varphi_{2} \nabla \mathcal{N} \varphi\right) \\
& =-\left(\operatorname{div}\left(\nabla \varphi_{1} \otimes \nabla \varphi\right)+\operatorname{div}\left(\nabla \varphi \otimes \nabla \varphi_{2}\right), \Pi\left(\varphi_{2} \nabla \mathcal{N} \varphi\right)\right) .
\end{aligned}
$$

A further integration by parts together with the homogeneous Neumann boundary condition for $\varphi$ entails

$$
\begin{aligned}
& -\left(\operatorname{div}\left(\nabla \varphi_{1} \otimes \nabla \varphi\right), \Pi\left(\varphi_{2} \nabla \mathcal{N} \varphi\right)\right) \\
& =\left(\nabla \varphi_{1} \otimes \nabla \varphi, \nabla \Pi\left(\varphi_{2} \nabla \mathcal{N} \varphi\right)\right)-\int_{\partial \Omega}\left(\nabla \varphi \otimes \nabla \varphi_{1}\right) \Pi\left(\varphi_{2} \nabla \mathcal{N} \varphi\right) \cdot \mathbf{n} \mathrm{d} \sigma \\
& =\left(\nabla \varphi_{1} \otimes \nabla \varphi, \nabla \Pi\left(\varphi_{2} \nabla \mathcal{N} \varphi\right)\right)-\int_{\partial \Omega}(\nabla \varphi \cdot \mathbf{n})\left(\nabla \varphi_{1} \cdot \Pi\left(\varphi_{2} \nabla \mathcal{N} \varphi\right)\right) \mathrm{d} \sigma \\
& =\left(\nabla \varphi_{1} \otimes \nabla \varphi, \nabla \Pi\left(\varphi_{2} \nabla \mathcal{N} \varphi\right)\right):=J_{1} .
\end{aligned}
$$

Similarly, we infer that

$$
\begin{aligned}
& -\left(\operatorname{div}\left(\nabla \varphi \otimes \nabla \varphi_{2}\right), \Pi\left(\varphi_{2} \nabla \mathcal{N} \varphi\right)\right) \\
& \quad=\left(\nabla \varphi \otimes \nabla \varphi_{2}, \nabla \Pi\left(\varphi_{2} \nabla \mathcal{N} \varphi\right)\right)-\int_{\partial \Omega}\left(\nabla \varphi_{2} \cdot \mathbf{n}\right)\left(\nabla \varphi \cdot \Pi\left(\varphi_{2} \nabla \mathcal{N} \varphi\right)\right) \mathrm{d} \sigma \\
& \quad=\left(\nabla \varphi \otimes \nabla \varphi_{2}, \nabla \Pi\left(\varphi_{2} \nabla \mathcal{N} \varphi\right)\right):=J_{2} .
\end{aligned}
$$

We now estimate $J_{1}$ and $J_{2}$. Exploiting (2.6), (2.9) and (4.3), we obtain

$$
\begin{aligned}
J_{1} & \leq C\left\|\nabla \varphi_{1}\right\|_{\mathbf{L}^{4}(\Omega)}\|\nabla \varphi\|\left\|\nabla \Pi\left(\varphi_{2} \nabla \mathcal{N} \varphi\right)\right\|_{\mathbf{L}^{4}(\Omega)} \\
& \leq C\left\|\nabla \varphi_{1}\right\|^{\frac{1}{2}}\left\|\nabla \varphi_{1}\right\|_{\mathbf{V}}^{\frac{1}{2}}\|\nabla \varphi\|\left\|\nabla \Pi\left(\varphi_{2} \nabla \mathcal{N} \varphi\right)\right\|^{\frac{1}{2}}\left\|\nabla \Pi\left(\varphi_{2} \nabla \mathcal{N} \varphi\right)\right\|_{\mathbf{V}}^{\frac{1}{2}} \\
& \leq C\left\|\varphi_{1}\right\|_{H^{2}(\Omega)}^{\frac{1}{2}}\|\nabla \varphi\|\left\|\Pi\left(\varphi_{2} \nabla \mathcal{N} \varphi\right)\right\|_{\mathbf{V}}^{\frac{1}{2}}\left\|\Pi\left(\varphi_{2} \nabla \mathcal{N} \varphi\right)\right\|_{\mathbf{H}^{2}(\Omega)}^{\frac{1}{2}} \\
& \leq C\left\|\varphi_{1}\right\|_{H^{2}(\Omega)}^{\frac{1}{2}}\|\nabla \varphi\|\left\|\varphi_{2} \nabla \mathcal{N} \varphi\right\|_{\mathbf{V}}^{\frac{1}{2}}\left\|\varphi_{2} \nabla \mathcal{N} \varphi\right\|_{\mathbf{H}^{2}(\Omega)}^{\frac{1}{2}} .
\end{aligned}
$$


It follows from $(2.4),(2.5),(2.10),(2.12)$ and $(4.3)$, that

$$
\begin{aligned}
\left\|\varphi_{2} \nabla \mathcal{N} \varphi\right\|_{\mathbf{V}} & \leq C\left\|\varphi_{2}\right\|_{V}\|\nabla \mathcal{N} \varphi\|_{\mathbf{L}^{\infty}(\Omega)}+C\left\|\varphi_{2}\right\|_{L^{\infty}(\Omega)}\|\nabla \mathcal{N} \varphi\|_{\mathbf{V}} \\
& \leq C\|\nabla \mathcal{N} \varphi\|_{\mathbf{L}^{\infty}(\Omega)}+C\|\nabla \mathcal{N} \varphi\|_{\mathbf{V}} \\
& \leq C\|\nabla \mathcal{N} \varphi\|^{\frac{1}{2}}\|\nabla \mathcal{N} \varphi\|_{\mathbf{H}^{2}(\Omega)}^{\frac{1}{2}}+C\|\varphi\| \\
& \leq C\|\varphi\|_{V_{0}^{\prime}}^{\frac{1}{2}}\|\nabla \varphi\|^{\frac{1}{2}}
\end{aligned}
$$

On the other hand, by (2.5), (2.10), (2.14) and (4.3), we deduce that

$$
\begin{aligned}
\left\|\varphi_{2} \nabla \mathcal{N} \varphi\right\|_{\mathbf{H}^{2}(\Omega)} & \leq C\left\|\varphi_{2}\right\|_{H^{2}(\Omega)}\|\nabla \mathcal{N} \varphi\|_{\mathbf{L}^{\infty}(\Omega)}+C\left\|\varphi_{2}\right\|_{L^{\infty}(\Omega)}\|\nabla \mathcal{N} \varphi\|_{\mathbf{H}^{2}(\Omega)} \\
& \leq C\left\|\varphi_{2}\right\|_{H^{2}(\Omega)}\|\nabla \mathcal{N} \varphi\|^{\frac{1}{2}}\|\nabla \mathcal{N} \varphi\|_{\mathbf{H}^{2}(\Omega)}^{\frac{1}{2}}+C\|\nabla \mathcal{N} \varphi\|_{\mathbf{H}^{2}(\Omega)} \\
& \leq C\left\|\varphi_{2}\right\|_{H^{2}(\Omega)}\|\varphi\|_{V_{0}^{\prime}}^{\frac{1}{2}}\|\nabla \varphi\|^{\frac{1}{2}}+C\|\nabla \varphi\| .
\end{aligned}
$$

Thus, from (4.6) we obtain

$$
\begin{aligned}
J_{1} & \leq C\left\|\varphi_{1}\right\|_{H^{2}(\Omega)}^{\frac{1}{2}}\left\|\varphi_{2}\right\|_{H^{2}(\Omega)}^{\frac{1}{2}}\|\varphi\|_{V_{0}^{\prime}}^{\frac{1}{2}}\|\nabla \varphi\|^{\frac{3}{2}}+C\left\|\varphi_{1}\right\|_{H^{2}(\Omega)}^{\frac{1}{2}}\|\varphi\|_{V_{0}^{\prime}}^{\frac{1}{4}}\|\nabla \varphi\|^{\frac{7}{4}} \\
& :=R_{1}+R_{2} .
\end{aligned}
$$

Using Young's inequality, the reminder terms $R_{1}$ and $R_{2}$ can be controlled as follows

$$
\begin{aligned}
R_{1} & =C\left\|\varphi_{1}\right\|_{H^{2}(\Omega)}^{\frac{1}{2}}\left\|\varphi_{2}\right\|_{H^{2}(\Omega)}^{\frac{1}{2}}\|\varphi\|_{V_{0}^{\prime}}^{\frac{1}{2}}\|\nabla \varphi\|^{\frac{3}{2}} \\
& \leq \frac{1}{32}\|\nabla \varphi\|^{2}+C\left\|\varphi_{1}\right\|_{H^{2}(\Omega)}^{2}\left\|\varphi_{2}\right\|_{H^{2}(\Omega)}^{2}\|\varphi\|_{V_{0}^{\prime}}^{2},
\end{aligned}
$$

and

$$
\begin{aligned}
R_{2} & =C\left\|\varphi_{1}\right\|_{H^{2}(\Omega)}^{\frac{1}{2}}\|\varphi\|_{V_{0}^{\prime}}^{\frac{1}{4}}\|\nabla \varphi\|^{\frac{7}{4}} \\
& \leq \frac{1}{32}\|\nabla \varphi\|^{2}+C\left\|\varphi_{1}\right\|_{H^{2}(\Omega)}^{4}\|\varphi\|_{V_{0}^{\prime}}^{2} .
\end{aligned}
$$

Collecting the above estimates and using again Young's inequality, we end up with

$$
J_{1} \leq \frac{1}{16}\|\nabla \varphi\|^{2}+C\left(1+\left\|\varphi_{1}\right\|_{H^{2}(\Omega)}^{4}+\left\|\varphi_{2}\right\|_{H^{2}(\Omega)}^{4}\right)\|\varphi\|_{V_{0}^{\prime}}^{2}
$$

Repeating the same calculations for $J_{2}$ line by line, we get

$$
J_{2} \leq \frac{1}{16}\|\nabla \varphi\|^{2}+C\left(1+\left\|\varphi_{2}\right\|_{H^{2}(\Omega)}^{4}\right)\|\varphi\|_{V_{0}^{\prime}}^{2}
$$

Finally, combining (4.5) with the above controls of $I, J_{1}$ and $J_{2}$, we find the differential inequality

$$
\frac{1}{2} \frac{\mathrm{d}}{\mathrm{d} t}\|\varphi\|_{V_{0}^{\prime}}^{2}+\frac{1}{4}\|\nabla \varphi\|^{2} \leq C\left(1+\left\|\boldsymbol{u}_{1}\right\|^{2}+\left\|\varphi_{1}\right\|_{H^{2}(\Omega)}^{4}+\left\|\varphi_{2}\right\|_{H^{2}(\Omega)}^{4}\right)\|\varphi\|_{V_{0}^{\prime}}^{2}
$$

On the other hand, thanks to Theorem 2.1, we have

$$
\int_{0}^{T}\left(\left\|\boldsymbol{u}_{1}(\tau)\right\|^{2}+\left\|\varphi_{1}(\tau)\right\|_{H^{2}(\Omega)}^{4}+\left\|\varphi_{2}(\tau)\right\|_{H^{2}(\Omega)}^{4}\right) \mathrm{d} \tau \leq C .
$$

Thus an application of the Gronwall lemma together with (4.7) and (4.8) gives (2.22).

The proof of Theorem 2.3 is complete. 


\section{Regularity and Phase Separation in Two Dimensions}

In this section, we prove Theorem 2.4, which provides further regularity and the phase separation property of global finite energy weak solutions in two dimensions for $t>0$. The goal will be achieved by obtaining some higher-order estimates for the finite energy weak solutions that only depend on an up bound for the initial energy $\mathcal{E}\left(\varphi_{0}\right)$ and on the average of total mass $\bar{\varphi}_{0}$. In particular, these estimates will be independent of any other norm of $\varphi_{0}$. To this end, given arbitrary but fixed numbers $R>0$ and $m \in(-1,1)$, we consider global finite energy solutions $(\boldsymbol{u}, P, \varphi)$ departing from $\varphi_{0}$ with

$$
\mathcal{E}\left(\varphi_{0}\right) \leq R \quad \text { and } \quad \bar{\varphi}_{0}=m
$$

Consequently, in this section the generic constant $C>0$ depends on $R, m$ and possibly on $\Omega$.

Our first regularity result is the following regularity estimate for $\varphi_{t}$ :

Lemma 5.1. Let the assumptions of Theorem 2.4 hold. For any $\sigma>0$, there exists a positive constant $C=C(\sigma, R, m)$ such that

$$
\left\|\varphi_{t}\right\|_{L^{\infty}\left(\sigma, t ; V^{\prime}\right)}+\left\|\varphi_{t}\right\|_{L^{2}(t, t+1 ; V)} \leq C, \quad \forall t \geq \sigma .
$$

Proof. We first note that, by virtue of Theorem 2.2,

$$
\|\varphi(t)\|_{V}+\|\varphi(t)\|_{L^{\infty}(\Omega)} \leq C, \quad \forall t \geq 0 .
$$

Given $h>0$, let us introduce the difference quotient of a function by

$$
\partial_{t}^{h} f=\frac{1}{h}(f(t+h)-f(t)), \quad \forall t \geq 0 .
$$

Owing to Definition 2.1, the difference quotient of a finite energy weak solution satisfies

$$
\left\langle\left(\partial_{t}^{h} \varphi\right)_{t}, v\right\rangle+\left(\boldsymbol{u}(t+h) \cdot \nabla \partial_{t}^{h} \varphi, v\right)+\left(\partial_{t}^{h} \boldsymbol{u} \cdot \nabla \varphi(t), v\right)+\left(\nabla \partial_{t}^{h} \mu, \nabla v\right)=0, \quad \forall v \in V,
$$

for almost every $t \in(0,+\infty)$, where

$$
\partial_{t}^{h} \mu=-\Delta \partial_{t}^{h} \varphi+\frac{1}{h}\left(\Psi^{\prime}(\varphi(t+h))-\Psi^{\prime}(\varphi(t))\right)
$$

and

$$
\partial_{t}^{h} \boldsymbol{u}=-\Pi\left(\operatorname{div}\left(\nabla \varphi(t+h) \otimes \nabla \partial_{t}^{h} \varphi\right)+\operatorname{div}\left(\nabla \partial_{t}^{h} \varphi \otimes \nabla \varphi(t)\right)\right) .
$$

We observe from the mass conservation that

$$
\overline{\partial_{t}^{h} \varphi}=\partial_{t}^{h} \bar{\varphi}=0
$$

Taking $v=\mathcal{N} \partial_{t}^{h} \varphi$ in the above weak formulation, and exploiting (2.2), we have

$$
\begin{aligned}
& \frac{1}{2} \frac{\mathrm{d}}{\mathrm{d} t}\left\|\partial_{t}^{h} \varphi\right\|_{V_{0}^{\prime}}^{2}+\left(\partial_{t}^{h} \mu, \partial_{t}^{h} \varphi\right) \\
& \quad=-\left(\boldsymbol{u}(t+h) \cdot \nabla \partial_{t}^{h} \varphi, \mathcal{N} \partial_{t}^{h} \varphi\right)-\left(\partial_{t}^{h} \boldsymbol{u} \cdot \nabla \varphi(t), \mathcal{N} \partial_{t}^{h} \varphi\right)
\end{aligned}
$$


By the definition of $\partial_{t}^{h} \mu$ in (5.3) and making use of the homogeneous Neumann boundary condition for $\partial_{t}^{h} \varphi$ together with $(\mathrm{H})$ and (2.4), we get

$$
\begin{aligned}
\left(\partial_{t}^{h} \mu, \partial_{t}^{h} \varphi\right) & =\left\|\nabla \partial_{t}^{h} \varphi\right\|^{2}+\frac{1}{h}\left(\Psi^{\prime}(\varphi(t+h))-\Psi^{\prime}(\varphi(t)), \partial_{t}^{h} \varphi\right) \\
& \geq\left\|\nabla \partial_{t}^{h} \varphi\right\|^{2}-\alpha\left\|\partial_{t}^{h} \varphi\right\|^{2} \\
& \geq \frac{1}{2}\left\|\nabla \partial_{t}^{h} \varphi\right\|^{2}-C\left\|\partial_{t}^{h} \varphi\right\|_{V_{0}^{\prime}}^{2} .
\end{aligned}
$$

Setting

$$
K_{1}=\left(\boldsymbol{u}(t+h) \partial_{t}^{h} \varphi, \nabla \mathcal{N} \partial_{t}^{h} \varphi\right) \quad \text { and } \quad K_{2}=\left(\partial_{t}^{h} \boldsymbol{u} \varphi(t), \nabla \mathcal{N} \partial_{t}^{h} \varphi\right),
$$

we find the differential inequality from (5.5) such that

$$
\frac{1}{2} \frac{\mathrm{d}}{\mathrm{d} t}\left\|\partial_{t}^{h} \varphi\right\|_{V_{0}^{\prime}}^{2}+\frac{1}{2}\left\|\nabla \partial_{t}^{h} \varphi\right\|^{2} \leq C\left\|\partial_{t}^{h} \varphi\right\|_{V_{0}^{\prime}}^{2}+K_{1}+K_{2} .
$$

In order to control $K_{1}$ and $K_{2}$, we argue similarly to the proof of Theorem 2.3. By (2.1), (2.4), (2.5) and (2.9), we estimate $K_{1}$ as follows

$$
\begin{aligned}
K_{1} & \leq\|\boldsymbol{u}(t+h)\|\left\|\partial_{t}^{h} \varphi\right\|_{L^{4}(\Omega)}\left\|\nabla \mathcal{N} \partial_{t}^{h} \varphi\right\|_{\mathbf{L}^{4}(\Omega)} \\
& \leq C\|\boldsymbol{u}(t+h)\|\left\|\partial_{t}^{h} \varphi\right\|^{\frac{1}{2}}\left\|\partial_{t}^{h} \varphi\right\|_{V}^{\frac{1}{2}}\left\|\nabla \mathcal{N} \partial_{t}^{h} \varphi\right\|^{\frac{1}{2}}\left\|\nabla \mathcal{N} \partial_{t}^{h} \varphi\right\|_{\mathbf{V}}^{\frac{1}{2}} \\
& \leq C\|\boldsymbol{u}(t+h)\|\left\|\partial_{t}^{h} \varphi\right\|_{V_{0}^{\prime}}^{\frac{1}{2}}\left\|\partial_{t}^{h} \varphi\right\|\left\|\nabla \partial_{t}^{h} \varphi\right\|^{\frac{1}{2}} \\
& \leq C\|\boldsymbol{u}(t+h)\|\left\|\partial_{t}^{h} \varphi\right\|_{V_{0}^{\prime}}\left\|\nabla \partial_{t}^{h} \varphi\right\| \\
& \leq \frac{1}{8}\left\|\nabla \partial_{t}^{h} \varphi\right\|^{2}+C\|\boldsymbol{u}(t+h)\|^{2}\left\|\partial_{t}^{h} \varphi\right\|_{V_{0}^{\prime}}^{2}
\end{aligned}
$$

Regarding $K_{2}$, in light of (5.4) we obtain

$$
\begin{aligned}
K_{2}= & -\left(\Pi\left(\operatorname{div}\left(\nabla \varphi(t+h) \otimes \nabla \partial_{t}^{h} \varphi\right)\right), \varphi(t) \nabla \mathcal{N} \partial_{t}^{h} \varphi\right) \\
& -\left(\Pi\left(\operatorname{div}\left(\nabla \partial_{t}^{h} \varphi \otimes \nabla \varphi(t)\right)\right), \varphi(t) \nabla \mathcal{N} \partial_{t}^{h} \varphi\right) \\
= & -\left(\operatorname{div}\left(\nabla \varphi(t+h) \otimes \nabla \partial_{t}^{h} \varphi\right), \Pi\left(\varphi(t) \nabla \mathcal{N} \partial_{t}^{h} \varphi\right)\right) \\
& -\left(\operatorname{div}\left(\nabla \partial_{t}^{h} \varphi \otimes \nabla \varphi(t)\right), \Pi\left(\varphi(t) \nabla \mathcal{N} \partial_{t}^{h} \varphi\right)\right) \\
= & \left(\nabla \varphi(t+h) \otimes \nabla \partial_{t}^{h} \varphi, \nabla \Pi\left(\varphi(t) \nabla \mathcal{N} \partial_{t}^{h} \varphi\right)\right) \\
& +\left(\nabla \partial_{t}^{h} \varphi \otimes \nabla \varphi(t), \nabla \Pi\left(\varphi(t) \nabla \mathcal{N} \partial_{t}^{h} \varphi\right)\right) \\
:= & Z_{1}+Z_{2} .
\end{aligned}
$$

Let us proceed to estimate $Z_{1}$ and $Z_{2}$. By (2.6), (2.9) and (5.2), we deduce that

$$
\begin{aligned}
Z_{1} & \leq C\|\nabla \varphi(t+h)\|_{\mathbf{L}^{4}(\Omega)}\left\|\nabla \partial_{t}^{h} \varphi\right\|\left\|\nabla \Pi\left(\varphi(t) \nabla \mathcal{N} \partial_{t}^{h} \varphi\right)\right\|_{\mathbf{L}^{4}(\Omega)} \\
& \leq C\|\nabla \varphi(t+h)\|^{\frac{1}{2}}\|\varphi(t+h)\|_{H^{2}(\Omega)}^{\frac{1}{2}}\left\|\nabla \partial_{t}^{h} \varphi\right\|\left\|\Pi\left(\varphi(t) \nabla \mathcal{N} \partial_{t}^{h} \varphi\right)\right\|_{\mathbf{W}^{1,4}(\Omega)} \\
& \leq C\|\varphi(t+h)\|_{H^{2}(\Omega)}^{\frac{1}{2}}\left\|\nabla \partial_{t}^{h} \varphi\right\|\left\|\varphi(t) \nabla \mathcal{N} \partial_{t}^{h} \varphi\right\|_{\mathbf{V}}^{\frac{1}{2}}\left\|\varphi(t) \nabla \mathcal{N} \partial_{t}^{h} \varphi\right\|_{\mathbf{H}^{2}(\Omega)}^{\frac{1}{2}} .
\end{aligned}
$$

A further application of (2.4), (2.6), (2.10), (2.12), (2.14) and (5.2) yields

$$
\left\|\varphi(t) \nabla \mathcal{N} \partial_{t}^{h} \varphi\right\|_{\mathbf{V}} \leq C\|\varphi(t)\|_{V}\left\|\nabla \mathcal{N} \partial_{t}^{h} \varphi\right\|_{\mathbf{L}^{\infty}(\Omega)}+C\|\varphi(t)\|_{L^{\infty}(\Omega)}\left\|\nabla \mathcal{N} \partial_{t}^{h} \varphi\right\|_{\mathbf{V}}
$$




$$
\begin{aligned}
& \leq C\left\|\nabla \mathcal{N} \partial_{t}^{h} \varphi\right\|^{\frac{1}{2}}\left\|\nabla \mathcal{N} \partial_{t}^{h} \varphi\right\|_{\mathbf{H}^{2}(\Omega)}^{\frac{1}{2}}+C\left\|\partial_{t}^{h} \varphi\right\|, \\
& \leq C\left\|\partial_{t}^{h} \varphi\right\|_{V_{0}^{\prime}}^{\frac{1}{2}}\left\|\nabla \partial_{t}^{h} \varphi\right\|^{\frac{1}{2}}
\end{aligned}
$$

and

$$
\begin{aligned}
& \left\|\varphi(t) \nabla \mathcal{N} \partial_{t}^{h} \varphi\right\|_{\mathbf{H}^{2}(\Omega)} \\
& \quad \leq C\|\varphi(t)\|_{H^{2}(\Omega)}\left\|\nabla \mathcal{N} \partial_{t}^{h} \varphi\right\|_{\mathbf{L}^{\infty}(\Omega)}+C\|\varphi(t)\|_{L^{\infty}(\Omega)}\left\|\nabla \mathcal{N} \partial_{t}^{h} \varphi\right\|_{\mathbf{H}^{2}(\Omega)} \\
& \quad \leq C\|\varphi(t)\|_{H^{2}(\Omega)}\left\|\nabla \mathcal{N} \partial_{t}^{h} \varphi\right\|^{\frac{1}{2}}\left\|\nabla \mathcal{N} \partial_{t}^{h} \varphi\right\|_{\mathbf{H}^{2}(\Omega)}^{\frac{1}{2}}+C\left\|\nabla \mathcal{N} \partial_{t}^{h} \varphi\right\|_{\mathbf{H}^{2}(\Omega)} \\
& \quad \leq C\|\varphi(t)\|_{H^{2}(\Omega)}\left\|\partial_{t}^{h} \varphi\right\|_{V_{0}^{\prime}}^{\frac{1}{2}}\left\|\nabla \partial_{t}^{h} \varphi\right\|^{\frac{1}{2}}+C\left\|\nabla \partial_{t}^{h} \varphi\right\| .
\end{aligned}
$$

Therefore, we learn that

$$
\begin{aligned}
Z_{1} \leq & C\|\varphi(t+h)\|_{H^{2}(\Omega)}^{\frac{1}{2}}\|\varphi(t)\|_{H^{2}(\Omega)}^{\frac{1}{2}}\left\|\partial_{t}^{h} \varphi\right\|_{V_{0}^{\prime}}^{\frac{1}{2}}\left\|\nabla \partial_{t}^{h} \varphi\right\|^{\frac{3}{2}} \\
& +C\|\varphi(t+h)\|_{H^{2}(\Omega)}^{\frac{1}{2}}\left\|\partial_{t}^{h} \varphi\right\|_{V_{0}^{\prime}}^{\frac{1}{4}}\left\|\nabla \partial_{t}^{h} \varphi\right\|^{\frac{7}{4}} \\
:= & Y_{1}+Y_{2} .
\end{aligned}
$$

By Young's inequality, we infer that

$$
\begin{aligned}
Y_{1} & =C\|\varphi(t+h)\|_{H^{2}(\Omega)}^{\frac{1}{2}}\|\varphi(t)\|_{H^{2}(\Omega)}^{\frac{1}{2}}\left\|\partial_{t}^{h} \varphi\right\|_{V_{0}^{\prime}}^{\frac{1}{2}}\left\|\nabla \partial_{t}^{h} \varphi\right\|^{\frac{3}{2}} \\
& \leq \frac{1}{32}\left\|\nabla \partial_{t}^{h} \varphi\right\|^{2}+C\|\varphi(t+h)\|_{H^{2}(\Omega)}^{2}\|\varphi(t)\|_{H^{2}(\Omega)}^{2}\left\|\partial_{t}^{h} \varphi\right\|_{V_{0}^{\prime}}^{2},
\end{aligned}
$$

and

$$
\begin{aligned}
Y_{2} & =C\|\varphi(t+h)\|_{H^{2}(\Omega)}^{\frac{1}{2}}\left\|\partial_{t}^{h} \varphi\right\|_{V_{0}^{\prime}}^{\frac{1}{4}}\left\|\nabla \partial_{t}^{h} \varphi\right\|^{\frac{7}{4}} \\
& \leq \frac{1}{32}\left\|\nabla \partial_{t}^{h} \varphi\right\|^{2}+C\|\varphi(t+h)\|_{H^{2}(\Omega)}^{4}\left\|\partial_{t}^{h} \varphi\right\|_{V_{0}^{\prime}}^{2}
\end{aligned}
$$

Hence, combining the above estimates together, we end up with

$$
Z_{1} \leq \frac{1}{16}\left\|\nabla \partial_{t}^{h} \varphi\right\|^{2}+C\left(1+\|\varphi(t+h)\|_{H^{2}(\Omega)}^{4}+\|\varphi(t)\|_{H^{2}(\Omega)}^{4}\right)\left\|\partial_{t}^{h} \varphi\right\|_{V_{0}^{\prime}}^{2}
$$

Arguing in the same way for $Z_{2}$, we also find

$$
Z_{2} \leq \frac{1}{16}\left\|\nabla \partial_{t}^{h} \varphi\right\|^{2}+C\left(1+\|\varphi(t)\|_{H^{2}(\Omega)}^{4}\right)\left\|\partial_{t}^{h} \varphi\right\|_{V_{0}^{\prime}}^{2}
$$

Then, collecting the estimates of $K_{1}$ and $K_{2}$, from (5.6) we deduce the following differential inequality

$$
\begin{aligned}
& \frac{1}{2} \frac{\mathrm{d}}{\mathrm{d} t}\left\|\partial_{t}^{h} \varphi\right\|_{V_{0}^{\prime}}^{2}+\frac{1}{4}\left\|\nabla \partial_{t}^{h} \varphi\right\|^{2} \\
& \quad \leq C\left(1+\|\boldsymbol{u}(t+h)\|^{2}+\|\varphi(t+h)\|_{H^{2}(\Omega)}^{4}+\|\varphi(t)\|_{H^{2}(\Omega)}^{4}\right)\left\|\partial_{t}^{h} \varphi\right\|_{V_{0}^{\prime}}^{2}
\end{aligned}
$$

On account of

$$
\left\|\partial_{t}^{h} \varphi\right\|_{L^{2}\left(t, t+1 ; V_{0}^{\prime}\right)} \leq\left\|\varphi_{t}\right\|_{L^{2}\left(t, t+1 ; V_{0}^{\prime}\right)}, \quad \forall t \geq 0
$$


and the dissipative estimate (cf. Theorem 2.2)

$$
\int_{t}^{t+1}\left(\|\varphi(\tau)\|_{H^{2}(\Omega)}^{4}+\|\nabla \mu(\tau)\|^{2}+\|\boldsymbol{u}(\tau)\|^{2}+\left\|\varphi_{t}(\tau)\right\|_{V_{0}^{\prime}}^{2}\right) \mathrm{d} \tau \leq C, \quad \forall t \geq 0,
$$

an application of the uniform Gronwall lemma entails the uniform bounds

$$
\left\|\partial_{t}^{h} \varphi\right\|_{L^{\infty}\left(\sigma, t ; V_{0}^{\prime}\right)}+\left\|\partial_{t}^{h} \varphi\right\|_{L^{2}(t, t+1 ; V)} \leq C, \quad \forall t \geq \sigma .
$$

Here, $C$ is a positive constant which depends on $\sigma>0$ but is independent of $h$. A final passage to the limit as $h \rightarrow 0^{+}$completes the proof.

Thanks to Lemma 5.1, we derive a preliminary higher-order estimate for $\varphi$ with respect to the spatial variable.

Lemma 5.2. Let the assumptions of Theorem 2.4 hold. For any $\sigma>0$, there exists a positive constant $C=C(\sigma, R, m)$ such that

$$
\|\varphi\|_{L^{\infty}\left(1, t ; H^{2}(\Omega)\right)} \leq C, \quad \forall t \geq \sigma .
$$

Proof. By [3, Theorem 1.1], we have for arbitrary $T \geq \sigma$

$$
H^{1}(\sigma, T ; V) \cap L^{2}\left(\sigma, T ; H^{2}(\Omega)\right) \hookrightarrow \mathcal{C}\left([\sigma, T], W^{1,4}(\Omega)\right) .
$$

Hence, in light of Theorem 2.1 and Lemma 5.1, we infer that

$$
\varphi \in \mathcal{C}\left([\sigma,+\infty), W^{1,4}(\Omega)\right)
$$

In order to get a uniform-in-time estimate, we recall that (cf. Theorem 2.2)

$$
\|\varphi\|_{H^{1}(t, t+1 ; V)}+\|\varphi\|_{L^{2}\left(t, t+1 ; H^{2}(\Omega)\right)} \leq C, \quad \forall t \geq \sigma,
$$

where $C$ is independent of $t$. Then, by the above result, we have

$$
\|\varphi(t)\|_{W^{1,4}(\Omega)} \leq C, \quad \forall t \in[\sigma, \sigma+1] .
$$

By the same argument replacing $\varphi(\cdot)$ with $\varphi(\cdot+n)$, for any $n \in \mathbb{N}$, we have

$$
\|\varphi(t+n)\|_{W^{1,4}(\Omega)} \leq C, \quad \forall t \in[\sigma, \sigma+1] \text { and } \forall n \in \mathbb{N},
$$

where $C$ is independent of $n$. This in turn gives the uniform estimate

$$
\|\varphi(t)\|_{W^{1,4}(\Omega)} \leq C, \quad \forall t \geq \sigma
$$

Next, taking $v=\mathcal{N}(\mu-\bar{\mu})$ in the weak formulation (2.17), we get

$$
\left\langle\varphi_{t}, \mathcal{N}(\mu-\bar{\mu})\right\rangle-(\boldsymbol{u} \varphi, \nabla \mathcal{N}(\mu-\bar{\mu}))+(\mu, \mu-\bar{\mu})=0
$$

We note that

$$
(\mu, \mu-\bar{\mu})=\|\mu-\bar{\mu}\|^{2} .
$$


Besides, by (2.5), (2.6), (2.13) and (5.9), we control the other two terms as follows

$$
\begin{aligned}
\left\langle\varphi_{t}, \mathcal{N}(\mu-\bar{\mu})\right\rangle & \leq\left\|\varphi_{t}\right\|_{V_{0}^{\prime}}\|\mathcal{N}(\mu-\bar{\mu})\|_{V_{0}} \\
& \leq C\left\|\varphi_{t}\right\|_{V^{\prime}}\|\mu-\bar{\mu}\| \\
& \leq \frac{1}{4}\|\mu-\bar{\mu}\|^{2}+C\left\|\varphi_{t}\right\|_{V^{\prime}}^{2}
\end{aligned}
$$

and

$$
\begin{aligned}
(\boldsymbol{u} \varphi, \nabla \mathcal{N}(\mu-\bar{\mu})) & =-(\Pi(\operatorname{div}(\nabla \varphi \otimes \nabla \varphi)), \varphi \nabla \mathcal{N}(\mu-\bar{\mu})) \\
& =-(\operatorname{div}(\nabla \varphi \otimes \nabla \varphi), \Pi(\varphi \nabla \mathcal{N}(\mu-\bar{\mu}))) \\
& =(\nabla \varphi \otimes \nabla \varphi, \nabla \Pi(\varphi \nabla \mathcal{N}(\mu-\bar{\mu}))) \\
& \leq C\|\nabla \varphi\|_{\mathbf{L}^{4}(\Omega)}^{2}\|\Pi(\varphi \nabla \mathcal{N}(\mu-\bar{\mu}))\|_{\mathbf{V}} \\
& \leq C\|\varphi \nabla \mathcal{N}(\mu-\bar{\mu})\|_{\mathbf{V}} \\
& \leq C\|\varphi\|_{\mathbf{W}^{1,4}(\Omega)}\|\nabla \mathcal{N}(\mu-\bar{\mu})\|_{\mathbf{L}^{4}(\Omega)}+C\left\|_{\varphi}\right\|_{L^{\infty}(\Omega)}\|\nabla \mathcal{N}(\mu-\bar{\mu})\|_{\mathbf{V}} \\
& \leq C\|\mu-\bar{\mu}\| \\
& \leq \frac{1}{4}\|\mu-\bar{\mu}\|^{2}+C
\end{aligned}
$$

Collecting the above estimates and using Lemma 5.1, we find

$$
\|\mu-\bar{\mu}\|_{L^{\infty}(\sigma, t ; H)} \leq C, \quad \forall t \geq \sigma .
$$

Applying Lemma 7.1 with $f=\mu+\Theta_{0} \varphi$, the above estimate entails

$$
\|\Delta \varphi\|_{L^{\infty}(\sigma, t ; H)} \leq C, \quad \forall t \geq \sigma .
$$

Finally, due to classical elliptic regularity results for the Neumann problem, we conclude that (5.8) holds. The proof is complete.

Now we can improve the regularity properties of global finite energy weak solutions $(\boldsymbol{u}, P, \varphi)$ on the time interval $[\sigma,+\infty)$ for any $\sigma>0$.

Lemma 5.3. Let the assumptions of Theorem 2.4 hold. For any $p>2$, there exists a positive constant $C=C(\sigma, p, R, m)$ such that

$$
\begin{array}{ll}
\|\mu\|_{L^{\infty}(\sigma, t ; V)}+\|\varphi\|_{L^{\infty}\left(\sigma, t ; W^{2, p}(\Omega)\right)} \leq C, & \forall t \geq \sigma, \\
\|\boldsymbol{u}\|_{L^{\infty}(\sigma, t ; \mathbf{V})}+\|P\|_{L^{\infty}\left(\sigma, t ; H^{2}(\Omega)\right)} \leq C, & \forall t \geq \sigma .
\end{array}
$$

Proof. First, we observe that

$$
\boldsymbol{u}=-\nabla P^{*}-\Delta \varphi \nabla \varphi, \quad \text { a.e. }(x, t) \in \Omega \times(0,+\infty),
$$

where $P^{*}$ is the modified pressure given by $P^{*}=P+\Psi(\varphi)$ (cf. Remark 2.2). Thus, we have $\boldsymbol{u}=\Pi(-\Delta \varphi \nabla \varphi)$ and by (2.6) together with the uniform estimates in Lemma 5.2, it follows that

$$
\|\boldsymbol{u}\|_{L^{\infty}\left(\sigma, t ; \mathbf{L}^{\frac{3}{2}}(\Omega)\right)} \leq C\|\Delta \varphi \nabla \varphi\|_{L^{\infty}\left(\sigma, t ; \mathbf{L}^{\frac{3}{2}}(\Omega)\right)}
$$




$$
\begin{aligned}
& \leq C\|\varphi\|_{L^{\infty}\left(\sigma, t ; H^{2}(\Omega)\right)}\|\varphi\|_{L^{\infty}\left(\sigma, t ; W^{1,6}(\Omega)\right)} \\
& \leq C .
\end{aligned}
$$

Next, we prove a uniform bound for the $V$-norm of $\mu$ arguing as in the proof of Theorem 2.1. As customary, we need to control its average value over $\Omega$. To this end, we recall that the singular potential $\Psi$ satisfies

$$
\left\|\Psi^{\prime}(\varphi)\right\|_{L^{1}(\Omega)} \leq C \int_{\Omega}\left(\varphi-\bar{\varphi}_{0}\right)\left(\Psi^{\prime}(\varphi)-\overline{\Psi^{\prime}(\varphi)}\right) \mathrm{d} x+C
$$

where $C$ depends on $m$ (cf. (3.14)). Testing $\mu$ by $\varphi-\bar{\varphi}_{0}$, integrating by parts and using (2.1) and (5.2), we easily get (cf. (3.15))

$$
\int_{\Omega}\left(\varphi-\overline{\varphi_{0}}\right)\left(\Psi^{\prime}(\varphi)-\overline{\Psi^{\prime}(\varphi)}\right) \mathrm{d} x \leq C(1+\|\nabla \mu\|)
$$

Combining the above inequalities, we are led to the inequality

$$
\left\|\Psi^{\prime}(\varphi)\right\|_{L^{1}(\Omega)} \leq C(1+\|\nabla \mu\|),
$$

which together with (3.13) gives

$$
\|\mu\|_{V} \leq C(1+\|\nabla \mu\|)
$$

Now, taking $v=\mu$ in (2.17), we have

$$
\|\nabla \mu\|^{2}=-\left\langle\varphi_{t}, \mu\right\rangle-(\boldsymbol{u} \cdot \nabla \varphi, \mu) .
$$

By (5.1), (5.8), (5.11) and (5.13), we get

$$
\begin{aligned}
\|\nabla \mu\|^{2} & \leq\left\|\varphi_{t}\right\|_{V^{\prime}}\|\mu\|_{V}+\|\boldsymbol{u}\|_{\mathbf{L}^{\frac{3}{2}}(\Omega)}\|\nabla \varphi\|_{\mathbf{L}^{6}(\Omega)}\|\mu\|_{L^{6}(\Omega)} \\
& \leq\left\|\varphi_{t}\right\|_{V^{\prime}}\|\mu\|_{V}+\|\boldsymbol{u}\|_{\mathbf{L}^{\frac{3}{2}(\Omega)}}\|\varphi\|_{H^{2}(\Omega)}\|\mu\|_{V} \\
& \leq C(1+\|\nabla \mu\|) .
\end{aligned}
$$

Hence, we infer from the above estimate, Young's inequality and (5.13) that

$$
\|\mu\|_{L^{\infty}(\sigma, t ; V)} \leq C, \quad \forall t \geq \sigma .
$$

Keeping (5.14) in mind, we can apply Lemma 7.4 again with $f=\mu+\Theta_{0} \varphi$. As a consequence, for any $p>2$, there exists $C>0$ such that

$$
\|\varphi\|_{L^{\infty}\left(\sigma, t ; W^{2, p}(\Omega)\right)} \leq C, \quad \forall t \geq \sigma .
$$

Therefore, combining (3.43), (3.44), (5.14), (5.15) and using the Gagliardo-Nirenberg inequality (3.41) for $d=2$, we have

$$
\|\boldsymbol{u}\|_{L^{\infty}(\sigma, t ; \mathbf{V})}+\|P\|_{L^{\infty}\left(\sigma, t ; H^{2}(\Omega)\right)} \leq C, \quad \forall t \geq \sigma
$$

The proof is complete. 
Remark 5.1. Thanks to the regularity achieved in Lemma 5.3, it is easily seen that (2.17) holds almost everywhere in $\Omega \times(\sigma,+\infty)$ and, in particular, $\mu$ satisfies $\partial_{n} \mu=0$ almost everywhere on $\partial \Omega \times(\sigma,+\infty)$. Since $\sigma>0$ is arbitrary, we infer that any global weak solution with finite energy to problem (1.1)-(1.2) becomes a global strong solution instantaneously when $t>0$.

We now have the necessary ingredients to prove the validity of the strict separation property. The main task is to show that $S^{\prime}(\varphi)$ is essentially bounded in time and space. For this purpose, we adapt the strategy devised in [19]. In particular, higher-order estimates will be derived assuming further conditions on the singular potentials, which are still satisfied, e.g., by the logarithmic potential (1.4).

Lemma 5.4. Let the assumptions of Theorem 2.4 hold. Assume, in addition, that $S^{\prime \prime}$ is convex and satisfies

$$
\left|S^{\prime \prime}(s)\right| \leq e^{C\left|S^{\prime}(s)\right|+C}, \quad \forall s \in(-1,1),
$$

where $C$ is a positive constant. Then, for any $\sigma>0$, there exists $C=C(\sigma, R, m)>0$ such that

$$
\left\|\varphi_{t}\right\|_{L^{\infty}(2 \sigma, t ; H)}+\|\mu\|_{L^{\infty}\left(2 \sigma, t ; H^{2}(\Omega)\right)} \leq C, \quad \forall t \geq 2 \sigma
$$

and there exists $\delta>0$ such that

$$
\|\varphi(t)\|_{L^{\infty}(\Omega)} \leq 1-\delta, \quad \forall t \geq 2 \sigma
$$

Moreover,

$$
\|\boldsymbol{u}\|_{L^{\infty}\left(2 \sigma, t ; \mathbf{H}^{2}(\Omega)\right)}+\|P\|_{L^{\infty}\left(2 \sigma, t ; H^{3}(\Omega)\right)}+\|\varphi\|_{L^{\infty}\left(2 \sigma, t ; H^{4}(\Omega)\right)} \leq C, \quad \forall t \geq 2 \sigma .
$$

Proof. Under the assumption (5.16), we can apply Lemma 7.4 with $f=\mu+\Theta_{0} \varphi$. Hence, for any $p \geq 2$, using the estimate obtained in Lemma 5.3, there exists $C=C(p)$ such that

$$
\left\|S^{\prime \prime}(\varphi)\right\|_{L^{\infty}\left(\sigma, t ; L^{p}(\Omega)\right)} \leq C, \quad \forall t \geq \sigma .
$$

We recall that the finite difference $\partial_{t}^{h} \varphi$ solves

$$
\left\langle\left(\partial_{t}^{h} \varphi\right)_{t}, v\right\rangle+\left(\boldsymbol{u}(t+h) \cdot \nabla \partial_{t}^{h} \varphi, v\right)+\left(\partial_{t}^{h} \boldsymbol{u} \cdot \nabla \varphi(t), v\right)+\left(\nabla \partial_{t}^{h} \mu, \nabla v\right)=0, \quad \forall v \in V,
$$

where $\partial_{t}^{h} \mu$ and $\partial_{t}^{h} \boldsymbol{u}$ are given by (5.3) and (5.4), respectively. Taking $v=\partial_{t}^{h} \varphi$, we get

$$
\frac{1}{2} \frac{\mathrm{d}}{\mathrm{d} t}\left\|\partial_{t}^{h} \varphi\right\|^{2}+\left(\nabla \partial_{t}^{h} \mu, \nabla v\right)=H_{1}+H_{2}
$$

having set

$$
H_{1}=-\left(\boldsymbol{u}(t+h) \cdot \nabla \partial_{t}^{h} \varphi, \partial_{t}^{h} \varphi\right), \quad H_{2}=-\left(\partial_{t}^{h} \boldsymbol{u} \cdot \nabla \varphi(t), \partial_{t}^{h} \varphi\right)
$$

By integration by parts and making use of the homogeneous Neumann boundary conditions for $\partial_{t}^{h} \varphi$ and $\partial_{t}^{h} \mu$, we find

$$
\left(\nabla \partial_{t}^{h} \mu, \nabla v\right)=-\left(\partial_{t}^{h} \mu, \Delta \partial_{t}^{h} \varphi\right)
$$




$$
=\left\|\Delta \partial_{t}^{h} \varphi\right\|^{2}-\Theta_{0}\left\|\nabla \partial_{t}^{h} \varphi\right\|^{2}-\left(\frac{1}{h}\left(S^{\prime}(\varphi(t+h))-S^{\prime}(\varphi(t))\right), \Delta \partial_{t}^{h} \varphi\right) .
$$

By the convexity of $S^{\prime \prime}$, we argue as in [19] to obtain

$$
\begin{aligned}
\frac{1}{h}\left|S^{\prime}(\varphi(t+h))-S^{\prime}(\varphi(t))\right| & \leq \int_{0}^{1} S^{\prime \prime}(\tau \varphi(t+h)+(1-\tau) \varphi(t))\left|\partial_{t}^{h} \varphi\right| \mathrm{d} \tau \\
& \leq\left(S^{\prime \prime}(\varphi(t+h))+S^{\prime \prime}(\varphi(t))\right)\left|\partial_{t}^{h} \varphi\right|
\end{aligned}
$$

and as a consequence, it follows that

$$
\begin{aligned}
& \left|\left(\frac{1}{h}\left(S^{\prime}(\varphi(t+h))-S^{\prime}(\varphi(t))\right), \Delta \partial_{t}^{h} \varphi\right)\right| \\
& \quad \leq \frac{1}{2}\left\|\Delta \partial_{t}^{h} \varphi\right\|^{2}+C\left(\left\|S^{\prime \prime}(\varphi(t+h))\right\|_{L^{3}(\Omega)}^{2}+\left\|S^{\prime \prime}(\varphi(t))\right\|_{L^{3}(\Omega)}^{2}\right)\left\|\partial_{t}^{h} \varphi\right\|_{L^{6}(\Omega)}^{2}
\end{aligned}
$$

By (2.1) and the boundary conditions, we infer from Poincaré's inequality and integration by parts that

$$
\left\|\partial_{t}^{h} \varphi\right\|_{L^{6}(\Omega)}^{2} \leq C\left\|\nabla \partial_{t}^{h} \varphi\right\|^{2} \leq C\left\|\partial_{t}^{h} \varphi\right\|\left\|\Delta \partial_{t}^{h} \varphi\right\| .
$$

Therefore, we easily derive from (5.20) the differential inequality

$$
\begin{aligned}
& \frac{1}{2} \frac{\mathrm{d}}{\mathrm{d} t}\left\|\partial_{t}^{h} \varphi\right\|^{2}+\frac{1}{4}\left\|\Delta \partial_{t}^{h} \varphi\right\|^{2} \\
& \quad \leq C\left(1+\left\|S^{\prime \prime}(\varphi(t+h))\right\|_{L^{3}(\Omega)}^{4}+\left\|S^{\prime \prime}(\varphi(t))\right\|_{L^{3}(\Omega)}^{4}\right)\left\|\partial_{t}^{h} \varphi\right\|^{2}+H_{1}+H_{2}
\end{aligned}
$$

Regarding the term $H_{1}$, by Lemma 5.3, the Sobolev embedding $V \hookrightarrow L^{p}(\Omega)$ for any $p \geq 1$, and the elliptic estimate for the Neumann problem, we get

$$
\begin{aligned}
H_{1} & \leq\|\boldsymbol{u}(t+h)\|_{\mathbf{L}^{6}(\Omega)}\left\|\nabla \partial_{t}^{h} \varphi\right\|_{\mathbf{L}^{3}(\Omega)}\left\|\partial_{t}^{h} \varphi\right\| \\
& \leq C\left\|\partial_{t}^{h} \varphi\right\|_{H^{2}(\Omega)}\left\|\partial_{t}^{h} \varphi\right\| \\
& \leq \frac{1}{16}\left\|\Delta \partial_{t}^{h} \varphi\right\|^{2}+C\left\|\partial_{t}^{h} \varphi\right\|^{2} .
\end{aligned}
$$

On the other hand, by the Darcy's equation (cf. Remark 2.2), Lemma 5.3, (2.13) and Poincaré's inequality, we infer that

$$
\begin{aligned}
H_{2} & =\left(\Pi\left(\operatorname{div}\left(\nabla \varphi(t+h) \otimes \nabla \partial_{t}^{h} \varphi+\nabla \partial_{t}^{h} \varphi \otimes \nabla \varphi(t)\right)\right), \nabla \varphi(t) \partial_{t}^{h} \varphi\right) \\
& =\left(\nabla \varphi(t+h) \otimes \nabla \partial_{t}^{h} \varphi+\nabla \partial_{t}^{h} \varphi \otimes \nabla \varphi(t), \nabla \Pi\left(\nabla \varphi(t) \partial_{t}^{h} \varphi\right)\right) \\
& \leq\left(\|\nabla \varphi(t+h)\|_{\mathbf{L}^{\infty}(\Omega)}+\|\nabla \varphi(t)\|_{\mathbf{L}^{\infty}(\Omega)}\right)\left\|\nabla \partial_{t}^{h} \varphi\right\|\left\|\nabla \Pi\left(\nabla \varphi(t) \partial_{t}^{h} \varphi\right)\right\| \\
& \leq C\left\|\nabla \partial_{t}^{h} \varphi\right\|\left\|\nabla \varphi(t) \partial_{t}^{h} \varphi\right\|_{\mathbf{V}} \\
& \leq C\left\|\nabla \partial_{t}^{h} \varphi\right\|\left(\|\varphi(t)\|_{W^{2,4}(\Omega)}\left\|\partial_{t}^{h} \varphi\right\|_{L^{4}(\Omega)}+C\|\nabla \varphi(t)\|_{\mathbf{L}^{\infty}(\Omega)}\left\|\nabla \partial_{t}^{h} \varphi\right\|\right) \\
& \leq C\left\|\nabla \partial_{t}^{h} \varphi\right\|^{2} \\
& \leq \frac{1}{16}\left\|\Delta \partial_{t}^{h} \varphi\right\|^{2}+C\left\|\partial_{t}^{h} \varphi\right\|^{2} .
\end{aligned}
$$

Collecting the above estimates for $H_{1}$ and $H_{2}$, we end up with

$$
\frac{1}{2} \frac{\mathrm{d}}{\mathrm{d} t}\left\|\partial_{t}^{h} \varphi\right\|^{2}+\frac{1}{8}\left\|\Delta \partial_{t}^{h} \varphi\right\|^{2} \leq W\left\|\partial_{t}^{h} \varphi\right\|^{2}
$$


where

$$
W(t)=C\left(1+\left\|S^{\prime \prime}(\varphi(t+h))\right\|_{L^{3}(\Omega)}^{4}+\left\|S^{\prime \prime}(\varphi(t))\right\|_{L^{3}(\Omega)}^{4}\right) .
$$

On account of the estimate (5.19) we have

$$
\int_{t}^{t+1} W(\tau) \mathrm{d} \tau \leq C, \quad \forall t \geq \sigma .
$$

Thus an application of the uniform Gronwall lemma implies that

$$
\left\|\partial_{t}^{h} \varphi\right\|_{L^{\infty}(2 \sigma, t ; H)}+\left\|\Delta \partial_{t}^{h} \varphi\right\|_{L^{2}(t, t+1 ; H)} \leq C, \quad \forall t \geq 2 \sigma,
$$

where the constant $C$ is independent of $h$. Passing to the limit as $h \rightarrow 0^{+}$, we obtain

$$
\left\|\varphi_{t}\right\|_{L^{\infty}(2 \sigma, t ; H)} \leq C, \quad \forall t \geq 2 \sigma .
$$

Now, using Lemma 5.3 and (5.22), we deduce by comparison that

$$
\|\mu\|_{L^{\infty}\left(2 \sigma, t ; H^{2}(\Omega)\right)} \leq C, \quad \forall t \geq 2 \sigma .
$$

Therefore, Lemma 7.2 together with the Sobolev embedding theorem yields

$$
\left\|S^{\prime}(\varphi)\right\|_{L^{\infty}\left(2 \sigma, t ; L^{\infty}(\Omega)\right)} \leq C, \quad \forall t \geq 2 \sigma .
$$

Due to the singularity of $S^{\prime}$ at the pure states \pm 1 , the above estimate implies the conclusion (5.17).

Thus, it is readily seen from (5.23) and the separation property (5.17) that

$$
\|\varphi\|_{L^{\infty}\left(2 \sigma, t ; H^{4}(\Omega)\right)} \leq C, \quad \forall t \geq 2 \sigma .
$$

Finally, by (2.14), (5.23) and (5.24), we arrive at

$$
\|\mu \nabla \varphi\|_{L^{\infty}\left(2 \sigma, t ; \mathbf{H}^{2}(\Omega)\right)} \leq C, \quad \forall t \geq 2 \sigma
$$

which gives (5.18) thanks to (2.6). The proof of is complete.

In summary, we have

Proof of Theorem 2.4. Combining the results obtained in Lemma 5.3 and Lemma 5.4, we immediately arrive at our conclusions in Theorem 2.4.

Remark 5.2. The validity of the separation property (5.17) is crucial, since it entails further regularity of weak finite energy solutions to problem (1.1)-(1.2). If (5.17) holds along the trajectory of solution $\varphi(t)$, then the singular potential $S(\varphi(t))$ is confined to an interval that does not contain the pure states \pm 1 and thus it is smooth. 


\section{Lyapunov Stability and Long-time Behavior}

As mentioned in the Introduction, when the spatial dimensional is three, the existence of a unique global strong solution to problem (1.1)-(1.2) with arbitrary large regular initial datum $\varphi_{0}$ can not be expected (cf. [34] for the case with regularity potential). In this section, we first prove the existence of a unique local strong solution $(\boldsymbol{u}, P, \varphi)$. Then we show that if the initial datum $\varphi_{0}$ is sufficiently close to a local minimizer of the energy functional $\mathcal{E}$, then the local strong solution is indeed a global one and $\varphi$ will stay close to that minimizer for all $t \geq 0$. Finally, we investigate the long-time behavior of global bounded solutions and show the uniqueness of asymptotic limit as $t \rightarrow+\infty$.

\subsection{Local strong solutions in three dimensions}

Theorem 6.1 (Local strong solutions). Let $d=3$. Assume that (H)-(H1) are satisfied and $\varphi_{0} \in H^{3}(\Omega)$ satisfying $\left\|\varphi_{0}\right\|_{C(\bar{\Omega})} \leq 1-\delta_{0}$, for an arbitrary but fixed $\delta_{0} \in(0,1)$, and $\partial_{n} \varphi=0$ on $\partial \Omega$. Then, there exists a unique local strong solution $(\boldsymbol{u}, P, \varphi)$ to problem (1.1)-(1.2) such that

$$
\begin{aligned}
& \boldsymbol{u} \in \mathcal{C}\left(\left[0, T^{*}\right], \mathbf{H}_{\sigma}\right) \cap L^{2}\left(0, T^{*} ; \mathbf{H}^{3}(\Omega)\right), \\
& P \in \mathcal{C}\left(\left[0, T^{*}\right], V\right) \cap L^{2}\left(0, T^{*} ; H^{4}(\Omega)\right), \\
& \varphi \in \mathcal{C}\left(\left[0, T^{*}\right], H^{3}(\Omega)\right) \cap H^{1}\left(0, T^{*} ; V\right) \cap L^{2}\left(0, T^{*} ; H^{5}(\Omega)\right), \\
& \mu \in \mathcal{C}\left(\left[0, T^{*}\right], V\right) \cap H^{1}\left(0, T^{*} ; V^{\prime}\right) \cap L^{2}\left(0, T^{*} ; H^{3}(\Omega)\right)
\end{aligned}
$$

and

$$
\|\varphi(t)\|_{C(\bar{\Omega})} \leq 1-\frac{1}{2} \delta_{0}, \quad \forall t \in\left[0, T^{*}\right]
$$

for some $T^{*} \in(0,+\infty)$ depending on $\left\|\varphi_{0}\right\|_{H^{3}(\Omega)}$ and $\delta_{0}$. In particular, the strong solution satisfies (1.1) for almost every $(x, t) \in \Omega \times\left(0, T^{*}\right)$ and the boundary conditions $\partial_{n} P=$ $\partial_{n} \mu=0$ on $\partial \Omega \times\left(0, T^{*}\right)$.

Proof. For any $\varepsilon \in(0,1)$, we introduce a regular approximating potential $\widehat{\Psi}_{\varepsilon} \in \mathcal{C}^{4}(\mathbb{R})$, namely,

$$
\widehat{\Psi}_{\varepsilon}(s)=\widehat{S}_{\varepsilon}(s)-\frac{\Theta_{0}}{2} s^{2}, \quad \forall s \in \mathbb{R},
$$

where

$$
\widehat{S}_{\varepsilon}(s)= \begin{cases}\sum_{j=0}^{4} \frac{1}{j !} S^{(j)}(1-\varepsilon)[s-(1-\varepsilon)]^{j}, & \forall s \geq 1-\varepsilon, \\ S(s), & \forall s \in[-1+\varepsilon, 1-\varepsilon], \\ \sum_{j=0}^{4} \frac{1}{j !} S^{(j)}(-1+\varepsilon)[s-(-1+\varepsilon)]^{j}, & \forall s \leq-1+\varepsilon .\end{cases}
$$


Then we consider the following approximating problem (AP2)

$$
\left\{\begin{array}{l}
\boldsymbol{u}_{\varepsilon}=-\nabla P_{\varepsilon}+\mu_{\varepsilon} \nabla \varphi_{\varepsilon}, \\
\operatorname{div} \boldsymbol{u}_{\varepsilon}=0, \\
\partial_{t} \varphi_{\varepsilon}+\boldsymbol{u}_{\varepsilon} \cdot \nabla \varphi_{\varepsilon}=\Delta \mu_{\varepsilon}, \\
\mu_{\varepsilon}=-\Delta \varphi_{\varepsilon}+\widehat{\Psi}_{\varepsilon}^{\prime}\left(\varphi_{\varepsilon}\right),
\end{array} \quad \text { in } \Omega \times(0, T),\right.
$$

subject to the initial and boundary conditions (1.2) (with $\varphi, \mu$ being replaced by $\varphi_{\varepsilon}$ and $\mu_{\varepsilon}$, respectively).

For any given $\delta_{0} \in(0,1)$, by (H1) we can choose a sufficiently small $\varepsilon \in\left(0, \min \left\{\kappa, \frac{1}{4} \delta_{0}\right\}\right]$ such that $\widehat{\Psi}_{\varepsilon} \in \mathcal{C}^{4}(\mathbb{R})$ satisfies

$$
\widehat{\Psi}_{\varepsilon}(s) \geq \gamma s^{4}-C, \quad \forall s \in \mathbb{R},
$$

for some positive constants $\gamma$ and $C$ depending on $\varepsilon$. Local well-posedness of the approximating problem (AP2) easily follows from a standard Galerkin method as in [26,34]. In particular, by means of a differential inequality involving the $H^{3}(\Omega)$-norm of $\varphi$ (see [34]), it follows that there exists $T_{\varepsilon} \in(0,+\infty)$ depending on $\left\|\varphi_{0}\right\|_{H^{3}(\Omega)}, \varepsilon$ and $\Omega$ such that problem (AP2) admits a unique local strong solution $\left(\boldsymbol{u}_{\varepsilon}, P_{\varepsilon}, \varphi_{\varepsilon}\right)$ on $\left[0, T_{\varepsilon}\right]$. Then, in light of $\varphi_{\varepsilon} \in \mathcal{C}\left(\left[0, T^{*}\right], H^{3}(\Omega)\right)$ and the Sobolev embedding $H^{3}(\Omega) \hookrightarrow \mathcal{C}(\bar{\Omega})$, the continuity in time of $\varphi_{\varepsilon}$ implies that there exists $T^{*} \in\left(0, T_{\varepsilon}\right]$ such that

$$
\left\|\varphi_{\varepsilon}(t)\right\|_{\mathcal{C}(\bar{\Omega})} \leq 1-\frac{1}{2} \delta_{0}, \quad \forall t \in\left[0, T^{*}\right]
$$

Noting that, by the choice of $\varepsilon$ and the definition of $\widehat{\Psi}_{\varepsilon}$, it holds $\left.\widehat{\Psi}_{\varepsilon}\right|_{\left[-1+\frac{1}{2} \delta_{0}, 1-\frac{1}{2} \delta_{0}\right]}=\Psi$. Hence, $\left(\boldsymbol{u}_{\varepsilon}, P_{\varepsilon}, \varphi_{\varepsilon}\right)$ actually coincides with the strong solution $(\boldsymbol{u}, P, \varphi)$ to the original problem $(1.1)-(1.2)$ on $\left[0, T^{*}\right]$, which is unique and satisfies the separation property (6.1). The proof is complete.

Next, we derive a higher-order differential inequality for (local) strong solutions.

Lemma 6.1. Let $d=3$ and let the assumptions of Theorem 6.1 hold. Assume that $(\boldsymbol{u}, P, \varphi)$ is a strong solution to problem (1.1)-(1.2) on $[0, T]$. Define the functional

$$
\Lambda(t)=\|\nabla \mu(t)\|^{2}+\|\boldsymbol{u}(t)\|^{2} .
$$

Then, we have

$$
\frac{\mathrm{d}}{\mathrm{d} t} \Lambda(t) \leq C_{*}\left(1+\Lambda(t)^{\frac{7}{3}}\right)
$$

for almost every $t \in(0, T)$. Here, the constant $C_{*}$ only depends on $\Omega, \alpha, m$ and $\mathcal{E}\left(\varphi_{0}\right)$.

Proof. By the regularity properties of a strong solution (see Theorem 6.1), we infer that $\boldsymbol{u} \cdot \nabla \varphi \in L^{2}(0, T ; V)$. Thus, we test the third equation of $(1.1)$ by $\mu_{t}$ such that

$$
\frac{1}{2} \frac{\mathrm{d}}{\mathrm{d} t}\|\nabla \mu\|^{2}+\left\|\nabla \varphi_{t}\right\|^{2}+\int_{\Omega} \Psi^{\prime \prime}(\varphi)\left|\varphi_{t}\right|^{2} \mathrm{~d} x=-\int_{\Omega}(\boldsymbol{u} \cdot \nabla \varphi) \mu_{t} \mathrm{~d} x, \quad \text { a.e. in }(0, T) .
$$


Next, in light of Remark 2.2, for any $\boldsymbol{v} \in \mathbf{V}_{\sigma}$, we have

$$
\begin{aligned}
\left\langle\boldsymbol{u}_{t}, \boldsymbol{v}\right\rangle & =\int_{\Omega}(-\nabla P+\mu \nabla \varphi)_{t} \cdot \boldsymbol{v} \mathrm{d} x \\
& =-2 \int_{\Omega} \operatorname{div}\left(\nabla \varphi \otimes \nabla \varphi_{t}\right) \cdot \boldsymbol{v} \mathrm{d} x \\
& =2 \int_{\Omega}\left(\nabla \varphi \otimes \nabla \varphi_{t}\right): \nabla \boldsymbol{v} \mathrm{d} x \\
& \leq 2\|\nabla \varphi\|_{\mathbf{L}^{\infty}(\Omega)}\left\|\nabla \varphi_{t}\right\|\|\boldsymbol{v}\|_{\mathbf{V}_{\sigma}} \\
& \leq C\|\varphi\|_{H^{3}(\Omega)}\left\|\nabla \varphi_{t}\right\|\|\boldsymbol{v}\|_{\mathbf{V}_{\sigma}},
\end{aligned}
$$

which entails that $\boldsymbol{u}_{t} \in L^{2}\left(0, T ; \mathbf{V}_{\sigma}^{\prime}\right)$. Thus, differentiating the first equation of (1.1) with respect to time and testing the resulting equation by $\boldsymbol{u}$, we get

$$
\frac{1}{2} \frac{\mathrm{d}}{\mathrm{d} t}\|\boldsymbol{u}\|^{2}=\int_{\Omega} \mu_{t} \nabla \varphi \cdot \boldsymbol{u} \mathrm{d} x+\int_{\Omega} \mu \nabla \varphi_{t} \cdot \boldsymbol{u} \mathrm{d} x, \quad \text { a.e. in }(0, T) .
$$

Noting that, by $(\mathrm{H})$ and $(2.4)$, we have

$$
\begin{aligned}
\int_{\Omega} \Psi^{\prime \prime}(\varphi)\left|\varphi_{t}\right|^{2} \mathrm{~d} x & \geq-\alpha\left\|\varphi_{t}\right\|_{V_{0}^{\prime}}\left\|\nabla \varphi_{t}\right\| \\
& \geq-\frac{1}{2}\left\|\nabla \varphi_{t}\right\|^{2}-C\left\|\varphi_{t}\right\|_{V_{0}^{\prime}}^{2}
\end{aligned}
$$

Then adding (6.7) to (6.8), we infer that

$$
\frac{\mathrm{d}}{\mathrm{d} t} \Lambda+\left\|\nabla \varphi_{t}\right\|^{2} \leq C\left\|\varphi_{t}\right\|_{V_{0}^{\prime}}^{2}+2 \int_{\Omega} \mu \nabla \varphi_{t} \cdot \boldsymbol{u} \mathrm{d} x
$$

We deduce from (1.1) and the Hölder inequality that

$$
\left\|\varphi_{t}\right\|_{V_{0}^{\prime}} \leq\|\boldsymbol{u}\|+\|\nabla \mu\|
$$

On the other hand, by the Gagliardo-Nirenberg inequality, we obtain

$$
\begin{aligned}
\int_{\Omega} \mu \nabla \varphi_{t} \cdot \boldsymbol{u} \mathrm{d} x & =-\int_{\Omega} \varphi_{t} \boldsymbol{u} \cdot \nabla \mu \mathrm{d} x \\
& \leq\|\boldsymbol{u}\|\left\|\varphi_{t}\right\|_{L^{3}(\Omega)}\|\nabla \mu\|_{\mathbf{L}^{6}(\Omega)} \\
& \leq C\|\boldsymbol{u}\|\left\|\varphi_{t}\right\|^{\frac{1}{2}}\left\|\nabla \varphi_{t}\right\|^{\frac{1}{2}}(\|\Delta \mu\|+\|\mu\|) \\
& \leq C\|\boldsymbol{u}\|\left\|\varphi_{t}\right\|_{V_{0}^{\prime}}^{\frac{1}{4}}\left\|\nabla \varphi_{t}\right\|^{\frac{3}{4}}\left(\left\|\varphi_{t}\right\|+\|\boldsymbol{u} \cdot \nabla \varphi\|+\|\mu\|\right) \\
& :=W_{1}+W_{2}+W_{3} .
\end{aligned}
$$

The reminder terms $W_{i}, i=1,3$ can be controlled by (2.1), (2.4) and (6.10) as follows

$$
\begin{aligned}
W_{1} & =C\|\boldsymbol{u}\|\left\|\varphi_{t}\right\|_{V_{0}^{\prime}}^{\frac{1}{4}}\left\|\nabla \varphi_{t}\right\|^{\frac{3}{4}}\left\|\varphi_{t}\right\| \\
& \leq\|\boldsymbol{u}\|\left\|\varphi_{t}\right\|_{V_{0}^{\prime}}^{\frac{3}{4}}\left\|\nabla \varphi_{t}\right\|^{\frac{5}{4}} \\
& \leq \frac{1}{6}\left\|\nabla \varphi_{t}\right\|^{2}+C\|\boldsymbol{u}\|^{\frac{8}{3}}\left\|\varphi_{t}\right\|_{V_{0}^{\prime}}^{2}
\end{aligned}
$$




$$
\leq \frac{1}{6}\left\|\nabla \varphi_{t}\right\|^{2}+C \Lambda^{\frac{7}{3}}
$$

and

$$
\begin{aligned}
W_{3} & =C\|\boldsymbol{u}\|\left\|\varphi_{t}\right\|_{V_{0}^{\prime}}^{\frac{1}{4}}\left\|\nabla \varphi_{t}\right\|^{\frac{3}{4}}\|\mu\| \\
& \leq C\|\boldsymbol{u}\|\left\|\varphi_{t}\right\|_{V_{0}^{\prime}}^{\frac{1}{4}}\left\|\nabla \varphi_{t}\right\|^{\frac{3}{4}}(\|\nabla \mu\|+|\bar{\mu}|) \\
& \leq C\|\boldsymbol{u}\|\left\|\varphi_{t}\right\|_{V_{0}^{\prime}}^{\frac{1}{4}}\left\|\nabla \varphi_{t}\right\|^{\frac{3}{4}}\left(\left\|\Psi^{\prime}(\varphi)\right\|_{L^{1}(\Omega)}+\|\nabla \mu\|\right) \\
& \leq \frac{1}{6}\left\|\nabla \varphi_{t}\right\|^{2}+C\left(1+\Lambda^{\frac{7}{3}}\right) .
\end{aligned}
$$

Here, we have used the estimates (5.12) and Young's inequality. Concerning $W_{2}$, by the Gagliardo-Nirenberg inequality (3.41), Young's inequality and Lemma 7.4, we get

$$
\begin{aligned}
W_{2} & =C\|\boldsymbol{u}\|\left\|\varphi_{t}\right\|_{V_{0}^{\prime}}^{\frac{1}{4}}\left\|\nabla \varphi_{t}\right\|^{\frac{3}{4}}\|\boldsymbol{u} \cdot \nabla \varphi\| \\
& \leq C\|\boldsymbol{u}\|^{2}\left\|\varphi_{t}\right\|_{V_{0}^{\prime}}^{\frac{1}{4}}\left\|\nabla \varphi_{t}\right\|^{\frac{3}{4}}\|\nabla \varphi\|_{\mathbf{L}^{\infty}(\Omega)} \\
& \leq \frac{1}{6}\left\|\nabla \varphi_{t}\right\|^{2}+C\|\boldsymbol{u}\|^{\frac{16}{5}}\left\|\varphi_{t}\right\|_{V_{0}^{\prime}}^{\frac{2}{5}}\|\varphi\|_{L^{\infty}(\Omega)}^{\frac{8}{15}}\|\varphi\|_{W^{2,6}(\Omega)}^{\frac{16}{15}} \\
& \leq \frac{1}{6}\left\|\nabla \varphi_{t}\right\|^{2}+C\left(1+\Lambda^{\frac{7}{3}}\right) .
\end{aligned}
$$

Collecting the above estimates together, we deduce (6.6). The proof is complete.

Remark 6.1. It is worth mentioning that inequality (6.6) has been obtained without using the separation property (6.1) but only the regularity of strong solutions to problem (1.1)(1.2).

\subsection{The stationary points}

Keeping the evolution problem (1.1)-(1.2) in mind and setting $m=\bar{\varphi}_{0}$, its corresponding stationary problem reads as follows

$$
\begin{cases}-\Delta \psi+\Psi^{\prime}(\psi)=\overline{\Psi^{\prime}(\psi)}, & \text { in } \Omega \\ \partial_{\mathbf{n}} \psi=0, & \text { on } \partial \Omega \\ \bar{\psi}=m . & \end{cases}
$$

For any given $m \in(-1,1)$, we introduce the set of stationary points

$$
\mathcal{S}_{m}=\left\{\psi \in H^{2}(\Omega), \mathcal{E}(\psi)<+\infty: \psi \text { solves }(6.12)\right\}
$$

The following result has been proven in [2, Section 6] (cf. Lemma 7.2).

Proposition 6.1. The set $\mathcal{S}_{m}$ is nonempty. Every element $\psi \in \mathcal{S}_{m}$ is a critical point of $\mathcal{E}$. Moreover, for each $\psi \in \mathcal{S}_{m}$, there is a constant $\xi \in(0,1)$ such that

$$
|\psi(x)| \leq 1-\xi, \quad \forall x \in \bar{\Omega} .
$$


Next, on account of Proposition 6.1 and arguing as in [2, Proposition 6.3] (or [1, Proposition 3]), the following gradient inequality of Łojasiewicz-Simon type can be established. This inequality will be crucial in the study of stability and long-time behavior of problem (1.1)-(1.2).

Lemma 6.2 (Eojasiewicz-Simon inequality). Let $d=2,3$. Assume that $\Psi$ satisfies $(H)$ and $\Psi$ is real analytic on the open interval $(-1,1)$. For any $m \in(-1,1)$, let $\psi \in \mathcal{S}_{m}$. Then, there exist constants $\theta \in\left(0, \frac{1}{2}\right)$ and $\beta>0$ such that

$$
|\mathcal{E}(\varphi)-\mathcal{E}(\psi)|^{1-\theta} \leq\left\|-\Delta \varphi+\Psi^{\prime}(\varphi)-\overline{\Psi^{\prime}(\varphi)}\right\|
$$

whenever $\varphi \in H^{2}(\Omega)$ satisfying $\|\varphi-\psi\|_{H^{2}(\Omega)}<\beta, \bar{\varphi}=m$ and $\partial_{n} \varphi=0$ on $\partial \Omega$.

Finally, we provide a characterization on local energy minimizers of the functional $\mathcal{E}$ (cf. Definition 2.2).

Lemma 6.3. Let $\psi \in \mathcal{Z}_{m}$ be a local energy minimizer of $\mathcal{E}$. Then $\psi \in \mathcal{S}_{m}$ and it satisfies the separation property (6.14).

Proof. We consider the single Cahn-Hilliard equation with singular potential

$$
\begin{cases}\varphi_{t}=\Delta \mu, & \text { in } \Omega \times(0,+\infty), \\ \mu=-\Delta \varphi+\Psi^{\prime}(\varphi), & \text { in } \Omega \times(0,+\infty), \\ \partial_{\mathbf{n}} \mu=\partial_{\mathbf{n}} \varphi=0, & \text { on } \partial \Omega \times(0,+\infty), \\ \varphi(\cdot, 0)=\varphi_{0}, & \text { in } \Omega .\end{cases}
$$

It has been proved in $\left[2\right.$, Section 6] that for both $d=2,3$ and any $\varphi_{0} \in V$ with $\Psi\left(\varphi_{0}\right) \in$ $L^{1}(\Omega), \bar{\varphi}_{0}=m \in(-1,1)$, problem (6.16) admits a unique solution $\varphi(t)$, which defines a family of operators $\{G(t)\}_{t \geq 0}$ such that $G(t) \in C\left([0,+\infty) ; \mathcal{Z}_{m}\right), G(t) \varphi_{0}=\varphi(t)$, for all $t \geq 0$. Besides, $\varphi(t)$ regularizes instantaneously for positive time, e.g., $G(t) \varphi_{0} \in H^{2}(\Omega)$ for every $t>0$. Then $\{G(t)\}_{t \geq 0}$ is a dynamical system on $\mathcal{Z}_{m}$ in the sense of $[8$, Definition 9.1.1] and the energy functional $\mathcal{E}(\varphi): \mathcal{Z}_{m} \rightarrow \mathbb{R}$ is a strict Lyapunov function for $\{G(t)\}_{t \geq 0}$ (due to an energy identity similar to (2.20) with $\boldsymbol{u}=\mathbf{0}$ ).

Therefore, every local energy minimizer $\psi \in \mathcal{Z}_{m}$ must be a stationary point of the evolution problem (6.16), i.e., $G(t) \psi=\psi$ for all $t \geq 0$. On the other hand, due to the instantaneous regularity property of problem (6.16), it has been shown in [2, Section 6$]$ that the set of all stationary points is characterized by $\mathcal{S}_{m}$. As a consequence, we conclude that $\psi \in \mathcal{S}_{m}$ and $\psi$ fulfills (6.14) for some $\xi \in(0,1)$.

\subsection{Proof of Theorem 2.5}

The following relations will be used in the subsequent proof.

Lemma 6.4. Let $d=3$ and let the assumptions of Theorem 6.1 hold. Suppose that $(\boldsymbol{u}, P, \varphi)$ is a strong solution to problem (1.1)-(1.2) on $[0, T]$. Then, we have

$$
\|\boldsymbol{u}\| \leq\|\nabla \mu\|, \quad\|\nabla \mu\| \leq\|\varphi\|_{H^{3}(\Omega)}+\left\|\Psi^{\prime \prime}(\varphi)\right\|_{L^{\infty}(\Omega)}\|\nabla \varphi\|,
$$




$$
\|\varphi\|_{H^{3}(\Omega)} \leq \bar{C}\left(\|\nabla \mu\|+\left\|\Psi^{\prime \prime}(\varphi)\right\|_{L^{\infty}(\Omega)}\|\varphi\|_{V}+\|\varphi\|_{V}\right)
$$

where the positive constant $\bar{C}$ only depends on $\Omega$.

Proof. The first two conclusions are obvious. Next, by elliptic regularity, we have

$$
\begin{aligned}
\|\varphi\|_{H^{3}(\Omega)} & \leq C\left(\|\Delta \varphi\|_{V}+\|\varphi\|_{L^{2}(\Omega)}\right) \\
& \leq C\left(\|\nabla \mu\|+\left\|\Psi^{\prime \prime}(\varphi)\right\|_{L^{\infty}(\Omega)}\|\nabla \varphi\|+\|\varphi\|_{H^{2}(\Omega)}\right) \\
& \leq C\left(\|\nabla \mu\|+\left\|\Psi^{\prime \prime}(\varphi)\right\|_{L^{\infty}(\Omega)}\|\varphi\|_{V}\right)+C\|\varphi\|_{V}^{\frac{1}{2}}\|\varphi\|_{H^{3}(\Omega)}^{\frac{1}{2}} \\
& \leq \frac{1}{2}\|\varphi\|_{H^{3}(\Omega)}+C\left(\|\nabla \mu\|+\left\|\Psi^{\prime \prime}(\varphi)\right\|_{L^{\infty}(\Omega)}\|\varphi\|_{V}+\|\varphi\|_{V}\right),
\end{aligned}
$$

where $C$ only depends on $\Omega$.

Proof of Theorem 2.5. The proof mainly follows the idea in [34], where problem (1.1)(1.2) with the regular potential (1.5) was considered. However, here we meet an extra difficulty due the singular potential $\Psi$. One essential step is to prove a strict separation property away from the pure states \pm 1 uniformly for $t \geq 0$ along the trajectory of $\varphi(t)$.

For any given $m \in(-1,1)$, let $\psi \in \mathcal{S}_{m}$ be an arbitrary local energy minimizer of the free energy $\mathcal{E}$ such that (cf. Definition 2.2 and Lemma 6.3)

$$
\|\psi\|_{\mathcal{C}(\bar{\Omega})} \leq 1-\xi, \quad \text { and } \quad \mathcal{E}(\varphi) \geq \mathcal{E}(\psi) \text { for all } \varphi \in \mathcal{Z}_{m}:\|\varphi-\psi\|_{V}<\chi
$$

We note that the constants $\xi \in(0,1)$ and $\chi>0$ are fixed once $\psi$ is given. Since $\Psi$ is assumed to be real analytic on $(-1,1)$, then by the separation property $(6.14)$ and the classical elliptic regularity theorem, we have $\psi \in H^{k}(\Omega)(k \in \mathbb{N})$ provided that $\Omega$ is a domain of class $\mathcal{C}^{k}$.

Due to the Sobolev embedding theorem $H^{2}(\Omega) \hookrightarrow \mathcal{C}(\bar{\Omega})(d=3)$, it holds

$$
\|\varphi\|_{\mathcal{C}(\bar{\Omega})} \leq C_{S}\|\varphi\|_{H^{2}(\Omega)}
$$

meanwhile, by the Poincaré's inequality (3.13), we have

$$
\|\varphi\|_{H^{1}(\Omega)}^{2} \leq C_{\Omega}\left(\|\nabla \varphi\|^{2}+|\bar{\varphi}|^{2}\right)
$$

where $C_{S}$ and $C_{\Omega}$ are positive constants that only depend on $\Omega$.

Step 1. Bounds for the initial datum. We consider any initial datum $\varphi_{0} \in H^{3}(\Omega)$ with $\partial_{\mathbf{n}} \varphi=0$ on $\partial \Omega$ that satisfies

$$
\begin{aligned}
& \left\|\varphi_{0}\right\|_{H^{3}(\Omega)} \leq M, \quad \bar{\varphi}_{0}=m, \\
& \left\|\varphi_{0}-\psi\right\|_{H^{2}(\Omega)} \leq \eta,
\end{aligned}
$$

where $\eta \in(0,1)$ will be determined later and $M>0$ is given by $(6.28)$ below. The fact $\eta<1$ implies that

$$
\left\|\varphi_{0}\right\|_{H^{2}(\Omega)} \leq\|\psi\|_{H^{2}(\Omega)}+1
$$


Moreover, if we further require

$$
0<\eta<\min \left\{1, \frac{\xi}{3 C_{S}}\right\}
$$

it follows from (6.18) that

$$
\left\|\varphi_{0}\right\|_{\mathcal{C}(\bar{\Omega})} \leq\|\psi\|_{\mathcal{C}(\bar{\Omega})}+\left\|\varphi_{0}-\psi\right\|_{\mathcal{C}(\bar{\Omega})} \leq 1-\frac{2 \xi}{3} .
$$

We define the constants

$$
K_{1}=\max _{s \in\left[-1+\frac{\xi}{3}, 1-\frac{\xi}{3}\right]}\left|\Psi^{\prime \prime}(s)\right|, \quad K_{2}=\max _{s \in[-1,1]}|\Psi(s)| .
$$

Hence, it holds (cf. (6.24))

$$
\begin{aligned}
\mathcal{E}\left(\varphi_{0}\right) & \leq \frac{1}{2}\left\|\nabla \varphi_{0}\right\|^{2}+|\Omega| \max _{s \in[-1,1]}|\Psi(s)| \\
& \leq \frac{1}{2}\left(\|\psi\|_{H^{1}(\Omega)}+1\right)^{2}+|\Omega| K_{2} \\
& :=\gamma_{1}
\end{aligned}
$$

where $\gamma_{1}>0$ only depends on $\|\psi\|_{H^{1}(\Omega)}, \Omega, \Psi$, but is independent of $\varphi_{0}$. Next, denote

$$
\gamma_{2}=C_{\Omega}^{\frac{1}{2}}\left(2 \gamma_{1}+\Theta_{0}|\Omega|+1\right)^{\frac{1}{2}} .
$$

Then we take the constant $M$ in (6.20) to be a sufficiently large but fixed number such that

$$
M \geq \bar{C}\left[\left(K_{1}+1\right) \gamma_{2}+2\right],
$$

where the constant $\bar{C}$ is given in Lemma 6.4 .

In the sequel we denote by $C, C_{i}$ those constants that only depend on $\Omega$, the averaged mass $m$, norms of the local minimizer $\psi$, the function $\Psi$ and parameters like $\xi, \chi$. Specific dependence will be pointed out explicitly.

Step 2. Strong solution on a finite interval. On account of the assumptions (6.20), (6.23) and (6.28), it follows from Theorem 6.1 that there exists a unique local strong solution $(\boldsymbol{u}, P, \varphi)$ to problem $(1.1)-(1.2)$ on $\left[0, T_{1}\right]$ such that

$$
\|\varphi(t)\|_{\mathcal{C}(\bar{\Omega})} \leq 1-\frac{\xi}{3}, \quad \forall t \in\left[0, T_{1}\right]
$$

where $T_{1}$ depends, in particular, on $M$ and $\xi$. We infer from (6.20) and Lemma 6.4 that

$$
\Lambda(0)=\|\nabla \mu(0)\|^{2}+\|\boldsymbol{u}(0)\|^{2} \leq 2\left[M+K_{1}\left(\|\psi\|_{V}+1\right)\right]^{2}:=M_{1} .
$$

It follows from the higher-order inequality Lemma 6.1 and (6.26) that there exists $T_{2} \in$ $\left(0, T_{1}\right]$ depending on $M_{1}, \alpha, \Omega, m$ and $\|\psi\|_{V}$ such that

$$
\Lambda(t) \leq 2 M_{1}, \quad \forall t \in\left[0, T_{2}\right] .
$$


Besides, we set

$$
E_{0}=\frac{\min \left\{1, M_{1}\right\} T_{2}}{2}
$$

which is a number that characterizes the energy drop along the trajectory $\varphi(t)$ (cf. (6.42)). By the energy identity $(2.20)$, it holds $\mathcal{E}(\varphi(t)) \leq \mathcal{E}\left(\varphi_{0}\right)$ for $t \geq 0$. On the other hand, we infer from (6.29) that

$$
\mathcal{E}(\varphi(t)) \geq \frac{1}{2}\|\nabla \varphi(t)\|^{2}-\frac{\Theta_{0}}{2}|\Omega|, \quad \forall t \in\left[0, T_{2}\right] .
$$

Then, we deduce from Poincaré's inequality (6.19), (6.26) and (6.27) that

$$
\begin{aligned}
\|\varphi(t)\|_{V}^{2} & \leq C_{\Omega}\left(\|\nabla \varphi(t)\|^{2}+m^{2}\right) \\
& \leq C_{\Omega}\left(2 \mathcal{E}\left(\varphi_{0}\right)+\Theta_{0}|\Omega|+1\right) \\
& \leq\left(\gamma_{2}\right)^{2}, \quad \forall t \in\left[0, T_{2}\right],
\end{aligned}
$$

which together with Lemma 6.4, (6.29) and (6.31) yields

$$
\|\varphi(t)\|_{H^{3}(\Omega)} \leq \bar{C}\left[\sqrt{2 M_{1}}+\left(K_{1}+1\right) \gamma_{2}\right]:=M_{2}, \quad \forall t \in\left[0, T_{2}\right] .
$$

Step 3. Refined estimates. Our aim is to find a sufficiently small $\eta>0$ such that the local strong solution satisfies a uniform bound that is independent of the existence interval. If this is true, then we can extend the unique local strong solution obtained in Step 1 to be a global one on $[0,+\infty)$.

It follows from (1.3), (6.29) and (6.33) that

$$
\begin{aligned}
\mathcal{E}\left(\varphi_{0}\right)-\mathcal{E}(\varphi(t)) \leq & \frac{1}{2}\left\|\nabla\left(\varphi(t)+\varphi_{0}\right)\right\|\left\|\nabla\left(\varphi(t)-\varphi_{0}\right)\right\| \\
& +\max _{s \in\left[-1+\frac{\xi}{3}, 1-\frac{\xi}{3}\right]} \mid \Psi^{\prime}(s)\left\|\varphi(t)-\varphi_{0}\right\|_{L^{1}(\Omega)} \\
\leq & M_{3}\left\|\varphi(t)-\varphi_{0}\right\|_{V}, \quad \forall t \in\left[0, T_{2}\right]
\end{aligned}
$$

where the constant $M_{3}$ depends on $\gamma_{2},\|\psi\|_{V}, \Psi, \xi$ and $\Omega$.

For any $\epsilon>0$, let us now set

$$
\omega=\min \left\{1, \epsilon, \chi, \beta, \frac{\xi}{3 C_{S}}, \frac{2 E_{0}}{3 M_{3}}\right\}
$$

where $\beta>0$ is determined by Lemma 6.2 and $E_{0}$ is given in (6.32). We define

$$
T_{\eta}=\inf \left\{t>0:\|\varphi(t)-\psi\|_{H^{2}(\Omega)} \geq \omega\right\} \quad \text { for } \eta \in\left(0, \frac{\omega}{2}\right] .
$$

By (6.20) and continuity of the strong solution $\varphi(t)$ in $H^{2}(\Omega)$, it follows that $T_{\eta}>0$. Next, we claim that there exists at least a value of $\eta$ such that $T_{\eta} \geq T_{2}$. Indeed, by contradiction, we have that $T_{\eta}<T_{2}$ for all $\eta \in\left(0, \frac{\omega}{2}\right]$. As a consequence, we apply Lemma 6.2 to derive the following energy inequality on the interval $\left[0, T_{\eta}\right] \subset\left[0, T_{2}\right]$

$$
-\frac{d}{d t}[\mathcal{E}(\varphi(t))-\mathcal{E}(\psi)]^{\theta}=-\theta[\mathcal{E}(\varphi(t))-\mathcal{E}(\psi)]^{\theta-1} \frac{d}{d t} \mathcal{E}(\varphi(t))
$$




$$
\begin{aligned}
& \geq \frac{\theta\left(\|\nabla \mu\|^{2}+\|\boldsymbol{u}\|^{2}\right)}{\|\nabla \mu\|} \\
& \geq \frac{\theta}{2}(\|\boldsymbol{u}\|+\|\nabla \mu\|) \geq C_{1}\left\|\varphi_{t}\right\|_{V_{0}^{\prime}},
\end{aligned}
$$

where the constant $C_{1}$ depends on $\theta$ and $\Omega$.

Here and after, we shall always exclude the trivial case such that there is a $t_{0} \in\left[0, T_{\eta}\right]$ such that $\mathcal{E}\left(\varphi\left(t_{0}\right)\right)=\mathcal{E}(\psi)$. In that case, $\|\boldsymbol{u}(t)\|=\|\nabla \mu(t)\|=0$ for all $t \geq t_{0}$ by virtue of the energy identity (2.20) and the evolution stops. Thus, using (6.37) and recalling that $\mathcal{E}(\varphi(t))$ is nonincreasing and, by the choice of $\omega, \mathcal{E}(\varphi(t)) \geq \mathcal{E}(\psi)$ on $\left[0, T_{\eta}\right]$ (cf. Definition 2.2 ), we infer that (cf. (6.35))

$$
\int_{0}^{T_{\eta}}\left\|\varphi_{t}(\tau)\right\|_{V_{0}^{\prime}} \mathrm{d} \tau \leq C_{1}\left(\mathcal{E}\left(\varphi_{0}\right)-\mathcal{E}(\psi)\right)^{\theta} \leq C_{2}\left\|\varphi_{0}-\psi\right\|_{V}^{\theta}
$$

where $C_{2}$ depends on $C_{1},\|\psi\|_{V}, \Psi, \xi$ and $\Omega$. As a consequence, we obtain

$$
\begin{aligned}
\left\|\varphi\left(T_{\eta}\right)-\psi\right\|_{H^{2}(\Omega)} & \leq\left\|\varphi_{0}-\psi\right\|_{H^{2}(\Omega)}+\left\|\varphi\left(T_{\eta}\right)-\varphi_{0}\right\|_{H^{2}(\Omega)} \\
& \leq\left\|\varphi_{0}-\psi\right\|_{H^{2}(\Omega)}+C_{3}\left\|\varphi\left(T_{\eta}\right)-\varphi_{0}\right\|_{H^{3}(\Omega)}^{\frac{3}{4}}\left\|\varphi\left(T_{\eta}\right)-\varphi_{0}\right\|_{V_{0}^{\prime}}^{\frac{1}{4}} \\
& \leq\left\|\varphi_{0}-\psi\right\|_{H^{2}(\Omega)}+C_{3}\left(M+M_{2}\right)^{\frac{3}{4}}\left(\int_{0}^{T_{\eta}}\left\|\varphi_{t}(\tau)\right\|_{V_{0}^{\prime}} \mathrm{d} \tau\right)^{\frac{1}{4}} \\
& \leq\left\|\varphi_{0}-\psi\right\|_{H^{2}(\Omega)}+C_{3}\left(M+M_{2}\right)^{\frac{3}{4}} C_{2}^{\frac{1}{4}}\left\|\varphi_{0}-\psi\right\|_{H^{2}(\Omega)}^{\frac{\theta}{4}} .
\end{aligned}
$$

Choosing now

$$
\eta=\min \left\{\frac{\omega}{2},\left(\frac{\omega}{4 C_{2}^{\frac{1}{4}} C_{3}\left(M+M_{2}\right)^{\frac{3}{4}}}\right)^{\frac{4}{\theta}}\right\},
$$

we have $\left\|\varphi\left(T_{\eta}\right)-\psi\right\|_{H^{2}} \leq \frac{3}{4} \omega<\omega$, which yields a contradiction with the definition of $T_{\eta}$. As a consequence, for the above choice of $\eta$, it holds $T_{\eta} \geq T_{2}$ and we learn that

$$
\|\varphi(t)-\psi\|_{H^{2}(\Omega)} \leq \omega, \quad \forall t \in\left[0, T_{2}\right] .
$$

In turn, by (6.36) and (6.39) we obtain, for all $t \in\left[0, T_{2}\right]$,

$$
\begin{aligned}
& \|\varphi(t)\|_{\mathcal{C}(\bar{\Omega})} \leq\|\psi\|_{\mathcal{C}(\bar{\Omega})}+C_{S}\|\varphi(t)-\psi\|_{H^{2}(\Omega)} \leq 1-\frac{2 \xi}{3}, \\
& \left\|\varphi(t)-\varphi_{0}\right\|_{H^{2}(\Omega)} \leq\|\varphi(t)-\psi\|_{H^{2}(\Omega)}+\left\|\varphi_{0}-\psi\right\|_{H^{2}(\Omega)} \leq \frac{E_{0}}{M_{3}}
\end{aligned}
$$

Then we infer from the energy identity $(2.20),(6.35)$ and (6.41) that

$$
\int_{0}^{T_{2}} \Lambda(\tau) \mathrm{d} \tau=\mathcal{E}\left(\varphi_{0}\right)-\mathcal{E}\left(\varphi\left(T_{2}\right)\right) \leq E_{0}
$$

Step 4. Iteration argument. Due to the nonnegativity of the function $\Lambda$ and (6.42), there exists $t^{*} \in\left[\frac{1}{2} T_{2}, T_{2}\right]$ such that

$$
\Lambda\left(t^{*}\right) \leq \min \left\{1, M_{1}\right\}
$$


Then it follows from Lemma $6.4,(6.28)$ and (6.33) that

$$
\left\|\varphi\left(t^{*}\right)\right\|_{H^{3}(\Omega)} \leq \bar{C}\left[\sqrt{\min \left\{1, M_{1}\right\}}+\left(K_{1}+1\right) \gamma_{2}\right]<M .
$$

Now we easily see that $\varphi\left(t^{*}\right)$ satisfies the same bounds as for $\varphi_{0}$ (compare $(6.20),(6.24)$ with (6.40), (6.44)). Moreover, by (6.39) and the choice of $\omega, \varphi\left(t^{*}\right)$ satisfies (6.21) where the up bound for $\eta$ that was used in Step 2 (i.e., (6.23)) is satisfied. Thus, we can take $\varphi\left(t^{*}\right)$ as the new initial datum and solve the problem (1.1)-(1.2) as in Step 1 and Step 2 on $\left[t^{*}, t^{*}+T_{2}\right]$. Thanks to the uniqueness of strong solutions, this yields a local strong solution defined on the extended interval $\left[0, t^{*}+T_{2}\right]$. After that we repeat the argument in Step 3 on $\left[0, \frac{3}{2} T_{2}\right] \subset\left[0, t^{*}+T_{2}\right]$ to derive the same refined estimates $(6.39)-(6.42)$ on $\left[0, \frac{3}{2} T_{2}\right]$ under exactly the same choice of $\eta$ (i.e., (6.38)). Again, there exists $t^{* *} \in\left[T_{2}, \frac{3}{2} T_{2}\right]$ such that $\Lambda\left(t^{* *}\right) \leq \min \left\{1, M_{1}\right\}$. Then we can take $t^{* *}$ as the initial time to repeat the above procedure and extend the unique local strong solution to the extended interval $\left[0,2 T_{2}\right]$ with uniform estimates (6.34) and (6.39) on [0,2 $\left.T_{2}\right]$.

By iteration, we easily arrive at the conclusion of Theorem 2.5.

\subsection{Proof of Theorem 2.6}

First, we show the following preliminary convergence result (a similar situation on the Cahn-Hilliard-Navier-Stokes system with singular potential can be found in [1]):

Proposition 6.2. Let $d=2,3$ and let the assumptions of Theorem 6.1 hold. Assume that the initial averaged mass satisfies $\bar{\varphi}_{0}=m \in(-1,1)$ and $S$ is real analytic in $(-1,1)$. If $(\boldsymbol{u}, P, \varphi)$ is a global strong solution to problem (1.1)-(1.2) such that there exist $M>0$ and $\delta \in(0,1)$

$$
\|\varphi(t)\|_{H^{3}(\Omega)} \leq M, \quad\|\varphi(t)\|_{\mathcal{C}(\bar{\Omega})} \leq 1-\delta, \quad \forall t \geq 0
$$

Then $(\boldsymbol{u}(t), \varphi(t))$ converges to a certain equilibrium $\left(\mathbf{0}, \varphi_{\infty}\right)$ as $t \rightarrow+\infty$ with the following convergence rate

$$
\|\boldsymbol{u}(t)\|+\left\|\varphi(t)-\varphi_{\infty}\right\|_{H^{3}(\Omega)} \leq C(1+t)^{-\frac{\theta}{1-2 \theta}}, \quad \forall t \geq 0
$$

Here, $\varphi_{\infty} \in \mathcal{S}_{m} \cap H^{3}(\Omega)$ is a solution to the stationary Cahn-Hilliard equation (6.12), $C>0$ is a constant depending on $M,\left\|\varphi_{\infty}\right\|_{H^{3}(\Omega)}, \delta, \Psi$ and $\Omega, \theta \in\left(0, \frac{1}{2}\right)$ is a constant depending only on $\varphi_{\infty}$.

Proof. We observe that due to the assumption $(6.45), \Psi(\varphi(t))$ is confined on $[-1+\delta, 1-\delta]$ along the trajectory $\varphi(t)$ for $t \geq 0$ so that Lemma 6.2 can apply. Then the conclusion follows from the same argument as in [34] for the problem (1.1)-(1.2) with the regular potential (1.5).

We can now proceed to prove Theorem 2.6:

Proof of Theorem 2.6. In light of Theorem 2.4 for any global weak finite energy solution $(\boldsymbol{u}, P, \varphi)$ in two dimensions, since we are discussing its long-time behavior, we can consider our solution from a certain positive time on to deal with a (global) strong solution. By 
Theorem 2.4 (resp. Theorem 2.5, with $\epsilon$ sufficiently small, cf. (6.40)) for global strong solutions in two (resp. three) dimensions, we see that the assumptions made in Proposition 6.2 are fulfilled. As an immediate consequence, the conclusion in Theorem 2.6 holds.

\subsection{Remark on the exponential stability of the averaged mass}

Finally, we present a result on the conditional exponential stability of the averaged mass $\bar{\varphi}_{0}$ for problem (1.1)-(1.2). The same property has been obtain in [26] for the case with a regular potential given by (1.5). To this end, it follows from the assumptions $(\mathrm{H})-(\mathrm{H} 1)$ that there exists a constant $\rho \in(1-\kappa, 1)$ such that

$$
\Psi^{\prime \prime}(s)>0, \quad \forall s \in(-1,-\rho] \cup[\rho, 1),
$$

namely, $\Psi$ is convex on the corresponding interval. Based on this observation, we have

Theorem 6.2 (Exponential stability of constant states). Let $d=2,3$. Assume that (H)-(H1) are satisfied and that the initial datum $\varphi_{0} \in H^{2}(\Omega)$ satisfies $\partial_{\mathbf{n}} \varphi_{0}=0$ on $\partial \Omega$. Besides, suppose that the initial averaged mass $\bar{\varphi}_{0}$ lies outside the so-called spinodal region, namely, $\bar{\varphi}_{0}=m \in(-1,-\rho) \cup(\rho, 1)$. There exists $\delta \in(0,1)$ sufficiently small such that if $\left\|\varphi_{0}-\bar{\varphi}_{0}\right\|_{H^{2}(\Omega)}<\delta$, then problem (1.1)-(1.2) admits a unique global strong solution $(\boldsymbol{u}, P, \varphi)$ such that

$$
\left\|\varphi(t)-\bar{\varphi}_{0}\right\|_{V} \leq C e^{-\zeta t}, \quad \forall t \geq 0
$$

for some constants $C, \zeta>0$ independent of time.

Proof. Since $|m| \in(\rho, 1)$, we take the interval

$$
I_{m}=[m-l, m+l], \quad \text { with } \quad 0<l<\frac{1}{2} \min \{|m|-\rho, 1-|m|\},
$$

such that $\Psi^{\prime \prime}(s)>0$ for all $s \in I_{m}$. One can easily construct a function $F \in \mathcal{C}^{3}(\mathbb{R})$ such that $F(m)=0, F^{\prime}(m)=0, F^{\prime \prime}(s)=\Psi^{\prime \prime}(s)$ on $I_{m}, F^{\prime \prime}(s) \geq 0$ for $s \in \mathbb{R} \backslash I_{m}$ and $F^{(3)}$ is bounded in $\mathbb{R}$. After this construction, the conclusion of Theorem 6.2 can be obtained by using exactly the same argument as in [26, Section 9]. In brief, we first solve the original problem (1.1)-(1.2) with $\Psi$ replaced by the regular potential $F$ and with the same initial data, obtaining the exponential stability result (6.48). Then the key observation is that one can find a sufficiently small $\delta$ such that the global strong solution to the modified problem will stay close enough to the constant state $\bar{\varphi}_{0}$ in $H^{2}(\Omega)$ for $t \geq 0$ (thus, in $\mathcal{C}(\bar{\Omega})$ by the Sobolev embedding theorem). Hence, this global solution is confined in $I_{m}$ so that, by the definition of $F$, it actually coincides with the global strong solution to the original problem (1.1)-(1.2).

Remark 6.2. Theorem 6.2 indicates that constant solutions are locally asymptotically stable when they are outside the spinodal region. However, inside the spinodal region, the dynamics is much more complicated and similar results on exponential stability cannot be expected. Our previous results Theorem 2.5 and Theorem 2.6 provide a first step towards a more comprehensive understanding of the longtime behavior of single solutions. 


\section{Appendix}

We consider the following homogeneous Neumann elliptic problem with a singular nonlinear term

$$
\begin{cases}-\Delta u+S^{\prime}(u)=f, & \text { in } \Omega \\ \partial_{\mathbf{n}} u=0, & \text { on } \partial \Omega .\end{cases}
$$

Here, $S$ is defined in Subsection 2.2 and satisfies the assumptions stated therein. We now report some elliptic estimates satisfied by the solution to problem (7.1). In particular, we assume that $u \in H^{2}(\Omega)$ with $S^{\prime}(u) \in H$ and satisfies (7.1) for almost every $x \in \Omega$. The proofs of the following results can be found in $[1,19]$.

Lemma 7.1. Let $f \in L^{2}(\Omega)$. Then, we have

$$
\|\Delta u\| \leq 2\|f-\bar{f}\|
$$

Lemma 7.2. Let $f \in L^{\infty}(\Omega)$. Then, we have

$$
\left\|S^{\prime}(u)\right\|_{L^{\infty}(\Omega)} \leq\|f\|_{L^{\infty}(\Omega)} .
$$

Lemma 7.3. Let $f \in H^{1}(\Omega)$. Given $R>0$, assume that $\|\nabla u\| \leq R$. Then, we have

$$
\|\Delta u\| \leq R^{\frac{1}{2}}\|\nabla f\|^{\frac{1}{2}}
$$

Lemma 7.4. Let $f \in H^{1}(\Omega)$. Then, there exists a positive constant $C=C(p)$ such that

$$
\|u\|_{W^{2, p}(\Omega)}+\left\|S^{\prime}(u)\right\|_{L^{p}(\Omega)} \leq C\left(1+\|f\|_{V}\right),
$$

where $p=6$ if $d=3$ and for any $p \geq 2$ if $d=2$. In addition, assume that $d=2$ and $S$ satisfies

$$
\left|S^{\prime \prime}(s)\right| \leq e^{C\left|S^{\prime}(s)\right|+C}, \quad \forall s \in(-1,1),
$$

for some positive constant $C$. Then, for any $p \geq 1$, there exists a positive constant $C=C(p)$ such that

$$
\left\|S^{\prime \prime}(u)\right\|_{L^{p}(\Omega)} \leq C\left(1+e^{C\|f\|_{V}^{2}}\right)
$$

\section{Acknowledgments}

The first two authors are members of the Gruppo Nazionale per l'Analisi Matematica, la Probabilità e le loro Applicazioni (GNAMPA) of the Istituto Nazionale di Alta Matematica (INdAM). Part of this work was done while the first author was visiting the School of Mathematical Sciences at Fudan University whose hospitality is gratefully acknowledged. The third author is partially supported by NNSFC under the grant No. 11631011 and Shanghai Center for Mathematical Sciences of Fudan University. 


\section{References}

[1] H. Abels, On a diffuse interface model for two-phase flows of viscous, incompressible fluids with matched densities, Arch. Ration. Mech. Anal., 194 (2009), 463-506.

[2] H. Abels and M. Wilke, Convergence to equilibrium for the Cahn-Hilliard equation with a logarithmic free energy, Nonlinear Anal., 67 (2007), 3176-3193.

[3] H. Amann, Compact embedding of vector-valued Sobolev and Besov spaces, Glas. Mat., 35 (2000), 161-177.

[4] J.M. Ball, Continuity properties and global attractors of generalized semiflows and the Navier-Stokes equation, J. Nonlinear Sci. 7 (1997), 475-502 (Erratum, J. Nonlinear Sci., 8 (1998), 233).

[5] S. Bosia, M. Conti and M. Grasselli, On the Cahn-Hilliard-Brinkman system, Commun. Math. Sci., 13 (2015), 1541-1567.

[6] H. Brézis, Functional Analysis, Sobolev Spaces and Partial Differential Equations, Universitext, Springer, New York, 2011.

[7] J.W. Cahn and J.E. Hilliard, Free energy of a nonuniform system I. Interfacial free energy, J. Chem. Phys., 2 (1958), 258-267.

[8] T. Cazenave and A. Haraux, An Introduction to Semilinear Evolution Equations, in: Oxford Lecture Series in Mathematics and its Applications, vol. 13, The Clarendon Press Oxford University Press, New York, 1998.

[9] M. Conti and A. Giorgini, On the Cahn-Hilliard-Brinkman system with singular potential and nonconstant viscosity, preprint 2016.

[10] M. Dai, E. Feireisl, E. Rocca, G. Schimperna and M.E. Schonbek, Analysis of a diffuse interface model of multispecies tumor growth, Nonlinearity, 30 (2017), 1639-1658.

[11] L. Dedé, H. Garcke and K.F. Lam, A Hele-Shaw-Cahn-Hilliard model for incompressible two-phase flows with different densities, MOX-Report NO.04/2017.

[12] F. Della Porta and M. Grasselli, On the nonlocal Cahn-Hilliard-Brinkman and CahnHilliard-Hele-Shaw systems, Commun. Pure Appl. Anal., 15 (2016), 299-317. Erratum, Commun. Pure Appl. Anal., 16 (2017), 369-372.

[13] M. Fei, Global sharp interface limit of the Hele-Shaw-Cahn-Hilliard system, Math. Methods Appl. Sci., 40 (2017), 833-852.

[14] X. Feng and S. Wise, Analysis of a Darcy-Cahn-Hilliard diffuse interface model for the Hele-Shaw flow and its fully discrete finite element approximation, SIAM J. Numer. Anal., 50 (2012), 1320-1343.

[15] S. Frigeri and M. Grasselli, Nonlocal Cahn-Hilliard-Navier-Stokes systems with singular potential, Dyn. Partial Differ. Equ., 24 (2012), 827-856.

[16] J. Geng and Z. Shen, The Neumann problem and Helmholtz decomposition in convex domains, J. Funct. Anal., 259 (2010) 2147-2164. 
[17] C.G. Gal, A. Giorgini and M. Grasselli, The nonlocal Cahn-Hilliard equation with singular potential: well-posedness, regularity and strict separation property, preprint 2016 .

[18] Y. Giga and T. Miyakawa, Solutions in $L^{r}$ of the Navier-Stokes initial value problem, Arch. Rational Mech. Anal., 89 (1985), 267-281.

[19] A. Giorgini, M. Grasselli and A. Miranville, Cahn-Hilliard--Oono equations with singular potentials, preprint 2016.

[20] V. Girault and P.A. Raviart, Finite Element Methods for Navier-Stokes Equations. Theory and Algorithms, Springer Series in Computational Mathematics, 5, SpringerVerlag, Berlin, 1986.

[21] D. Han, X. Wang and H. Wu, Existence and uniqueness of global weak solutions to a Cahn-Hilliard-Stokes-Darcy system for two phase incompressible flows in karstic geometry, J. Differential Equations, 257 (2014), 3887-3933.

[22] P.C. Hohenberg and B.I. Halperin, Theory of dynamic critical phenomena, Rev. Mod. Phys., 49 (1977), 435-479.

[23] J. Jiang, H. Wu and S. Zheng, Well-posedness and long-time behavior of a nonautonomous Cahn-Hilliard-Darcy system with mass source modeling tumor growth, J. Differential Equations, 259 (2015), 3032-3077.

[24] N. Kenmochi, M. Niezgódka and I. Pawlow, Subdifferential operator approach to the Cahn-Hilliard equation with constraint, J. Differential Equations, 117 (1995), $320-356$.

[25] H.-G. Lee, J.-S. Lowengrub and J. Goodman, Modeling pinch-off and reconnection in a Hele-Shaw cell. I. The models and their calibration, Phys. Fluids, 14 (2002), $492-512$.

[26] J. Lowengrub, E. Titi and K. Zhao, Analysis of a mixture model of tumor growth, European J. Appl. Math., 24 (2013), 691-734.

[27] S. Melchionna and E. Rocca, Varifold solutions of a sharp interface limit of a diffuse interface model for tumor growth, preprint 2016, arXiv:1610.04478.

[28] A. Miranville and S. Zelik, Robust exponential attractors for Cahn-Hilliard type equations with singular potentials, Math. Methods Appl. Sci., 27 (2004), 545-582.

[29] A. Miranville and S. Zelik, Attractors for dissipative partial differential equations in bounded and unbounded domains, in Handbook of Differential Equations, Evolutionary Partial Differential Equations, Vol. 4, C.M. Dafermos and M. Pokorny eds., Elsevier, Amsterdam, 103-200, 2008.

[30] E. Rocca and G. Schimperna, Universal attractor for some singular phase transition systems, Phys. D, 192 (2004), 279-307.

[31] P. Rybka and K.-H. Hoffmann, Convergence of solutions to Cahn-Hilliard equation, Commun. Partial Differential Equations, 24 (1999), 1055-1077.

[32] L. Simon, Asymptotics for a class of nonlinear evolution equation with applications to geometric problems, Ann. of Math., 118 (1983), 525-571. 
[33] R. Temam, Navier-Stokes Equations: Theory and Numerical Analysis, AMS, Providence, 2001.

[34] X. Wang and H. Wu, Long-time behavior for the Hele-Shaw-Cahn-Hilliard system, Asymptot. Anal., 78 (2012), 217-245.

[35] X. Wang and Z. Zhang, Well-posedness of the Hele-Shaw-Cahn-Hilliard system, Ann. Inst. H. Poincaré Anal. Non Linéaire, 30 (2013), 367-384.

[36] S.M. Wise, Unconditionally stable finite difference, nonlinear multigrid simulations of the Cahn-Hilliard-Hele-Shaw system of equations, J. Sci. Comput., 44 (2010), $38-68$. 